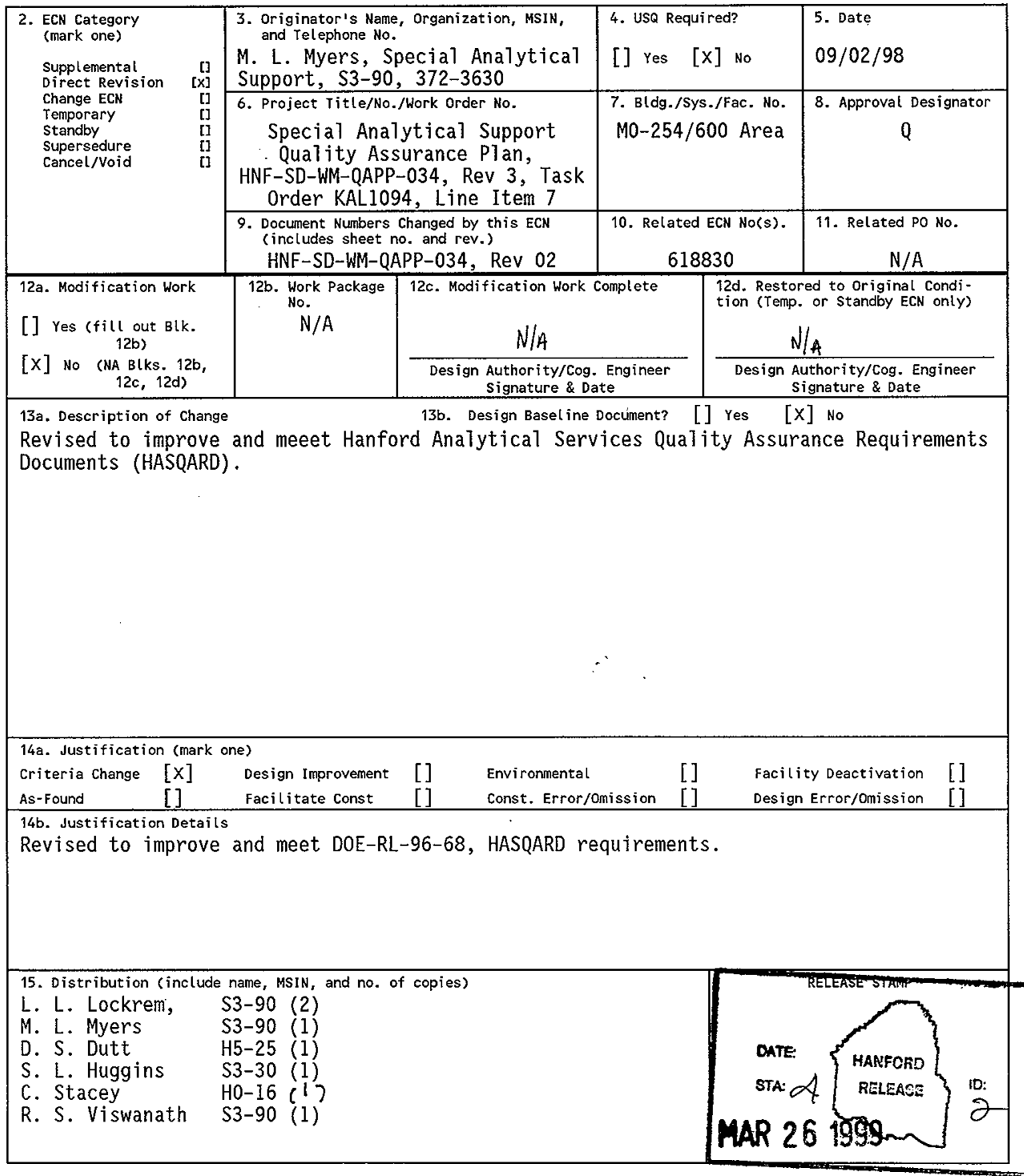




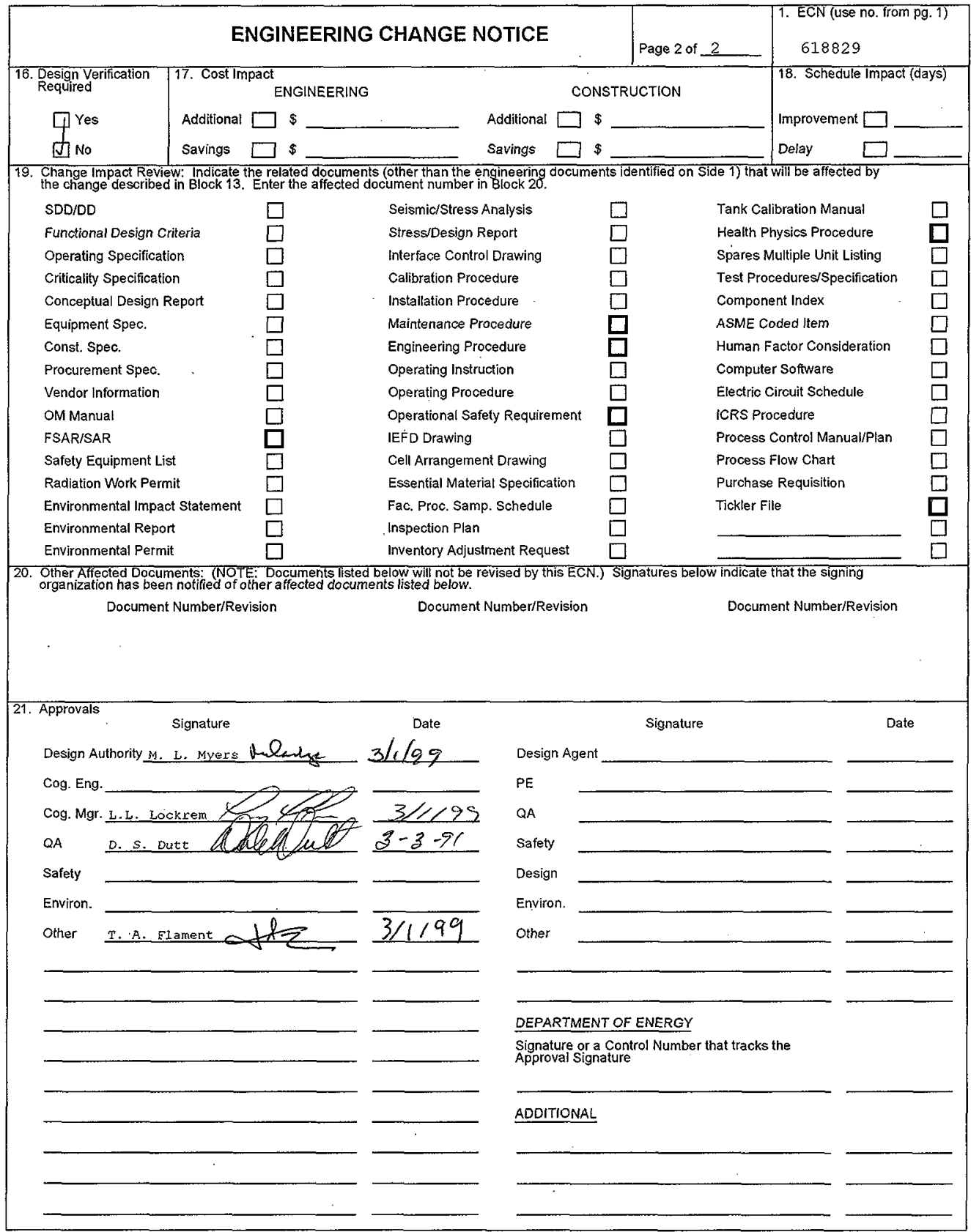




\section{Quality Assurance Management Plan (QAPP) Special Analytical Support (SAS)}

L. L. Lockrem

Numatec Hanford Corporation, Richland, WA 99352

U.S. Department of Energy Contract DE-AC06-96RL13200

ECN: 618829

EDT: 158942

Org Code: $8 \mathrm{C} 530$

B\&R Code: EW3130030

UC:

Charge Code: MADM1

Total Pages: $: 764$

Key Words:

Revised to improve and meet Hanford Analytical Services Quality Assurance Requirements Documents (HASQARD).

\section{Abstract:}

It is the policy of Special Analytical Support (SAS) that the analytical aspects of all environmental data generated and processed in the laboratory, subject to the Environmental Protection Agency (EPA), U.S. Department of Energy or other project specific requirements, be of known and acceptable quality. It is the intention of this QAPP to establish and assure that an effective quality controlled management system is maintained in order to meet the quality requirements of the intended use(s) of the data.

IRADEMARK DISCLAIMER. Reference herein to any specific comercial product, process, or service by trade name, trademark, manufacturer, or otherwise, does not necessarily constitute or imply its endorsement, recommendation, or favoring by the United States Government or any agency thereof or its contractors or subcontractors.

Printed in the United States of America. To obtain copies of this document, contact: Document Control Services, P.0. Box 950, Mailstop H6-08, Richland WA 99352, Phone (509) 372-2420;

Fax (509) 376-4989.
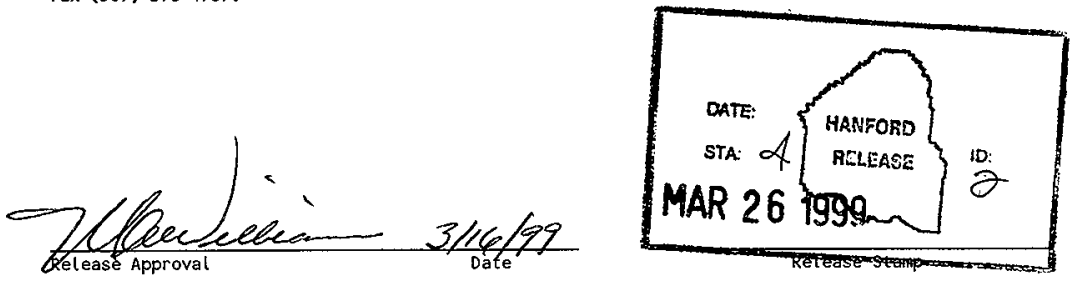

\section{Approved for Public Release}




\section{RECORD OF REVISION}

(1) Document Number

HNF-SD-WM-QAPP034

(2) Title

Quality Assurance Management PTan (QAPP) Special Analytical Support (SAS)

CHANGE CONTROL RECORD

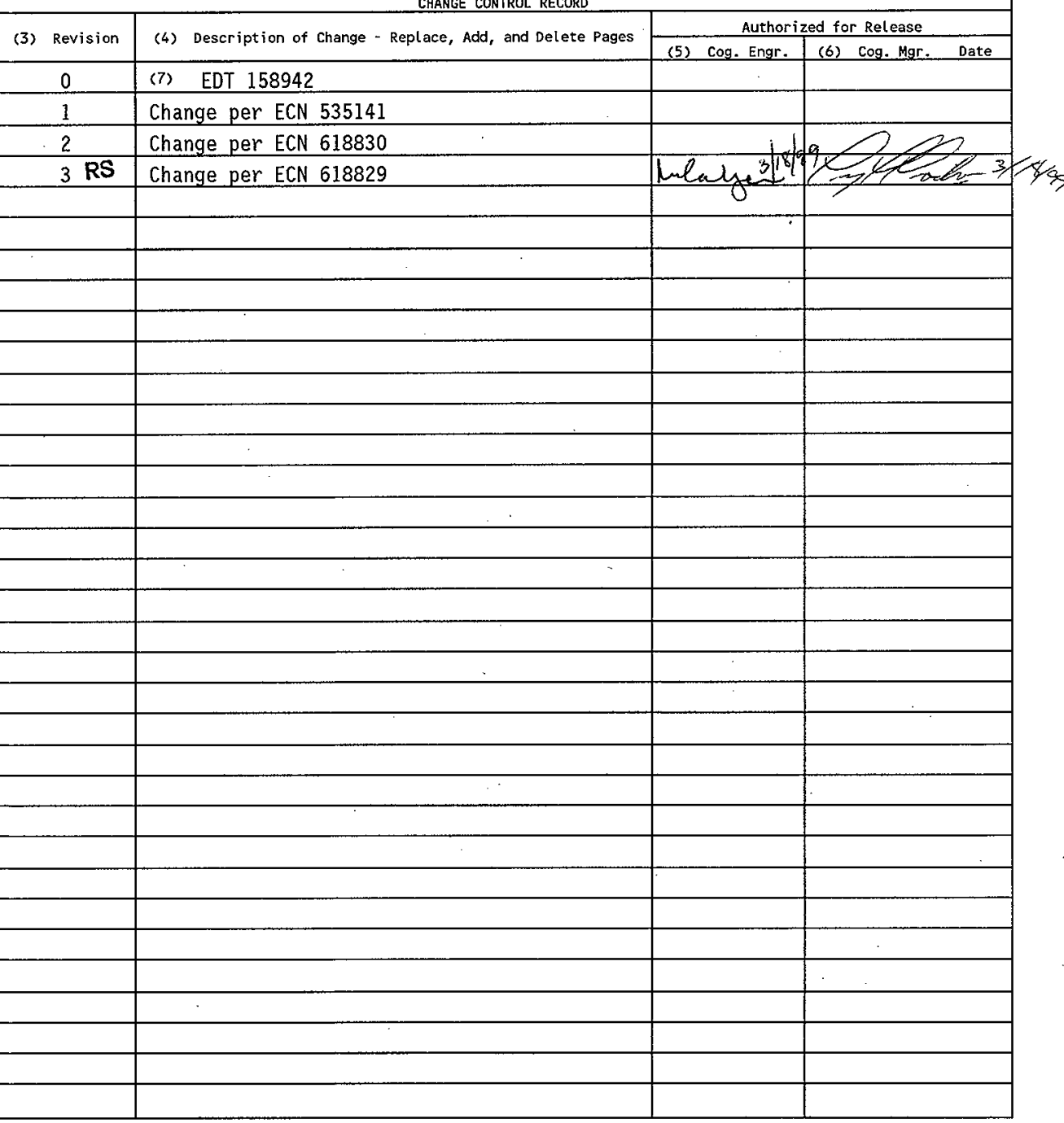




\title{
SPECIAL ANALYTICAL SUPPORT QUALITY ASSURANCE PLAN
}

\author{
HNF-SD-WM-QAPP-034 \\ Revision 3
}

\author{
Prepared by \\ M. L. Myers \\ Issued by \\ Special Analytical Support \\ Numatec Hanford Company
}




\title{
Special Analytical Support Quality Assurance Plan
}

\author{
Revision 3
}

September 1998

Approved:

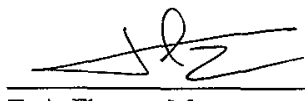

T. A. Flament,Manager

Numatec Hanford Company

$\frac{\text { Lul ha h }}{\text { M. L. Myers }}$

SAS Facility Quality Assurance Officer

Special Analytical Support

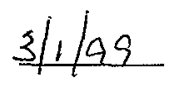

Date
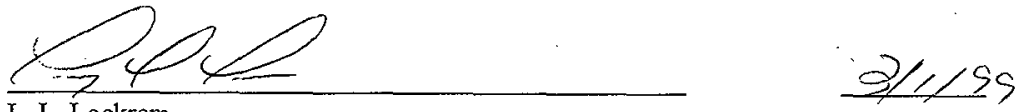

L. L. Lockrem

Manager, Special Analytical Support

Date

Numatec Hanford Company

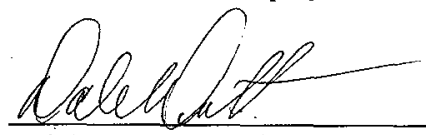

D. S. Dutt

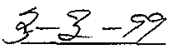

Date

Manager, ESH\&Q

Numatec Hanford Company 


\section{ACKNOWLEDGMENTS}

The completion of this document represents the efforts of the entire staff of Special Analytical Support. Special acknowledgment goes to Thomas Tribble for assisting SAS with ongoing quality improvement, Larry Markel for being kind enough to provide his expert advice, and various management personnel for their comments, concerns, and contributions.

Revision 3 of the Special Analytical Support Quality Assurance Project Plan is significantly different from Revision 2. The significant quality assurance and technical changes to these sections are summarized on the following pages.

Melanie Myers, Special Analytical Support, QA Officer

Numatec Hanford Company 


\section{EXPLANATION OF CHANGES TO HNF-SD-WM-QAPP-034}

The changes to this quality assurance program plan (QAPP) represent continual ongoing quality improvement of SAS. Revision 3 of this QAPP incorporates changes from the Hanford Analytical Services Quality Assurance Requirements Document (HASQARD) (Beyers and Grier 1997), general updates, process changes, and responses to assessments/audit issues that occurred in 1997 and 1998. It also meets the QA requirements of SAS customers. Changes from Westinghouse Hanford Company control manuals to HNF (Hanford) documents have been added as appropriate; this includes the change-over from HNF-CM-5-4 and all Laboratory Administrative Procedures (LAPts) matrixed to the corresponding sections of the new WMH-200 and WMH-310 manual sections.

In addition, this document meets the objectives of the Quality Assurance Program provided in the HNF-MP-599, Project Hanford Quality Assurance Program Description; HNF-SD-WM-QAPP-036, WMFSH Quality Assurance Program Plan (Jacobsen 1998); and HNF-2599, TRU Waste Isolation Pilot Program Quality Assurance Project Plan.

This document also has a completely revised format, revised Table of Contents, additions of organizational charts to clarify the matrix of SAS to Waste Management Hanford Laboratory, Waste Management Hanford, and.Fluor Daniel Hanford. Table 1-1 has been added as a cross reference reflecting how this QAPP meets the requirements of 10 CFR 830.120. Table 1-2 is a cross reference that reflects the HASQARD, DOE/RL-9698 requirements, as well as the applicable policies and procedures followed by SAS to implement these requirements.

Section 1 has been expanded to include the SAS ethical policies and mission statement.

Section 2 has been expanded to include more detail on personnel responsibilities and explain the interface with matrixed organizations. The table in this section is being updated to ensure that all SAS capabilities are listed.

Section 3 provides greater detail in the area of training and qualifications with the addition of minimal requirements for job-specific categories.

Precision, accuracy, completeness, comparability, and representativeness have been moved to this section with more detail.

Section 5 is the same except for adding that this section is used as a tool for the client in determining what data documentation and $\mathrm{QC}$ requirements are needed.

Systems Quality Assurance previously only covered software systems. Technical administrative systems and physical facility systems have been added.

A new section covering sampling methods as been added. SAS performs sampling on various waste matrixes. 
Sample Custody, Handling, and Storage has been expanded. This section now;covers Sample Receiving, Sample Log-in and Tracking, Sample Preservation, Internal Chain of Custody, and Sample Disposal and Waste Disposal.

The Calibration of Analytical Instruments previously included only Calibration Checks and Calibration Standards. This section now covers record keeping for calibration data, calibration of balances, thermometers, and pipettes, traceability and documentation of standards used in calibration, and calibration of laboratory measurement systems.

Section 8, which used to be the Analytical Test Methods section, is now the Laboratory Procedures section and explains the procedural requirements, technical and administrative procedures, preparation and review of supporting documents, document change control, new analytical methods, method modification, and qualification of analytical methods.

Data Reduction, Verification, and Reporting now includes data collection; and an explanation of significant figures and rounding under Data Reduction with ASTM rules. Information on data review also has been added. Data Reporting has been expanded to include the different types of data reporting formats, data reporting documentation, and data package structure.

The Records section covers generation of quality records, receipt control, records validation, corrections and records identification and indexing, maintenance and retention, retrieval and disposition, replacement, restoration or substitution of records, and turnover of records to the client.

The Quality Control section addresses general laboratory quality control and preparation techniques for inorganic and organic analysis.

All calculations have been moved to Section 12; discussions of detection limit considerations and control charts have been added.

Section 13, Assessments, has not changed a great deal.

Section 14 covers recommended preventive maintenance frequency for specific instruments.

Corrective actions and quality improvement were not included in the previous QAPP section hasThese topics have been added as Section 16.

Section 17, Quality Assurance Reports, has been added to this QAPP.

Data validation, data usability, and planning consideration also are new to this QAPP. They are covered in Section 18. 


\section{CONTENTS}

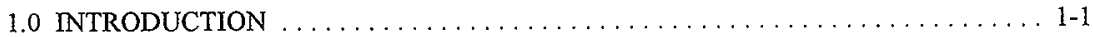

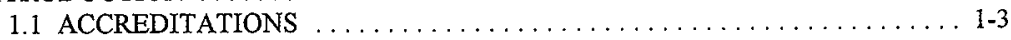

1.2 MISSION STATEMENT $\ldots \ldots \ldots \ldots \ldots \ldots \ldots \ldots \ldots \ldots \ldots \ldots \ldots, 1-3$

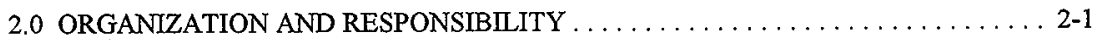

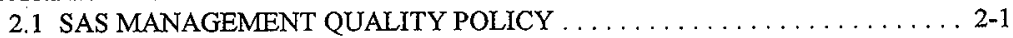

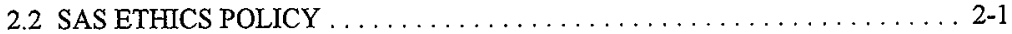

2.3 ORGANIZATIONAL RESPONSIBILITIES $\ldots \ldots \ldots \ldots \ldots \ldots \ldots \ldots . \ldots \ldots$

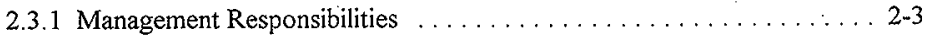

2.3.2 All SAS Personnel . . . . . . . . . . . . . . . . . . . .

2.3.3 Other Organizations $\ldots \ldots \ldots \ldots \ldots \ldots \ldots \ldots \ldots \ldots \ldots \ldots \ldots \ldots \ldots \ldots \ldots \ldots, 2-8$

3.0 PERSONNEL QUALIFICATION AND TRAINING $\ldots \ldots \ldots \ldots \ldots \ldots \ldots \ldots . . \ldots \ldots$

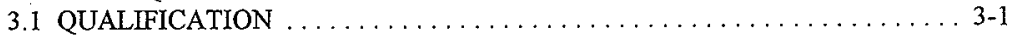

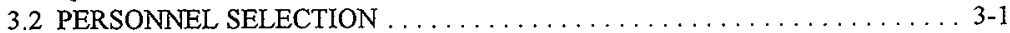

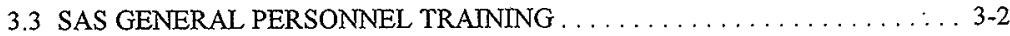

3.3.1 Chemists/Scientists . . . . . . . . . . . . . . . . . . . .

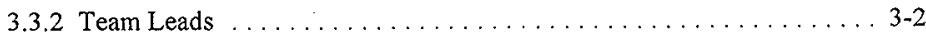

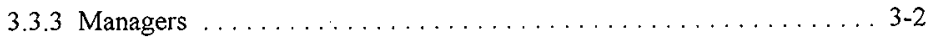

3.3.4 Additional Job-Specific Training . . . . . . . . . . . . . . . .

3.4 CONTINUING TRAINING REQUIREMENTS $\ldots \ldots \ldots \ldots \ldots \ldots \ldots \ldots .6,6$

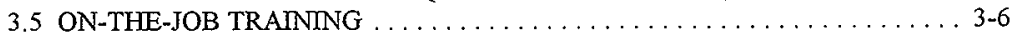

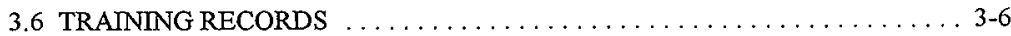

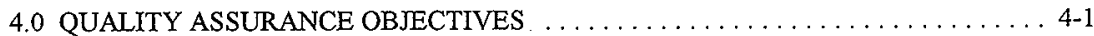

4.1 DATA QUALITY OBJECTIVES . . . . . . . . . . . . . . . 4-1

4.1.1 System for Notification of Unique Data Quality Requirements . . . . 4-2

4.1.2 Client Complaints and Resolution ................... 4-2

4.2 CLIENT DATA QUALITY REQUTREMENTS $\ldots \ldots \ldots \ldots \ldots \ldots \ldots .4-3$

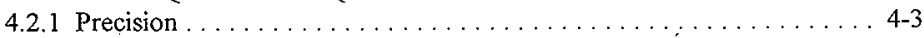

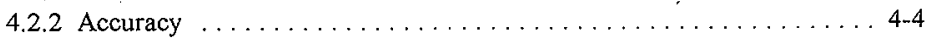

4.2 .3 Comparability ............................ 4-4

4.2 .4 Completeness . . . . . . . . . . . . . . . . . . . . . 4-4

4.2 .5 Representativeness ....................... 4-5

5.0 FIELD ANALYTICAL SUPPORT LEVELS $\ldots \ldots \ldots \ldots \ldots \ldots \ldots \ldots \ldots \ldots$ 5-1

6.0 SYSTEMS QUALITY ASSURANCE ...................... 6-1

6.1 SOFTWARE SYSTEMS QUALITY ASSURANCE $\ldots \ldots \ldots \ldots \ldots \ldots \ldots$ 6-1

6.1 .1 Control Requirements . . . . . . . . . . . . . . . . . . . .

6.1 .2 Acceptance Testing . . . . . . . . . . . . . . .

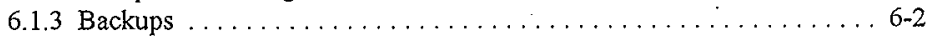

6.1 .4 User's Manuals . . . . . . . . . . . . . . . . . . . . $6-2$

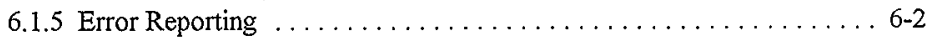


6.2 TECHNICAL/ADMMNISTRATIVE SYSTEMS $\ldots \ldots \ldots \ldots \ldots \ldots, \ldots, 2$

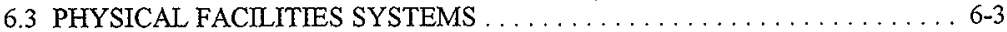

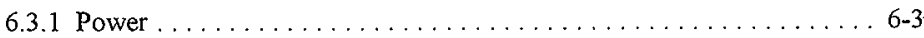

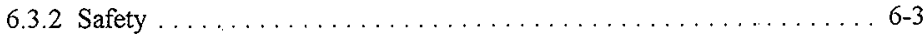

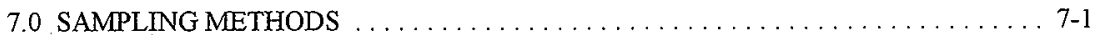

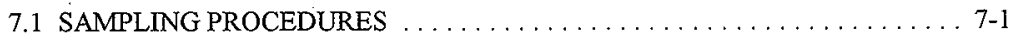

7.2 SAMPLE CUSTODY AND HANDLING $\ldots \ldots \ldots \ldots \ldots \ldots \ldots \ldots \ldots, 7-1$

7.2.1 Sample Security and Chain of Custody . . . . . . . . . . . . . 7

7.2.2 Sample Custodian ......................... $7-2$

7.3 HOLDING TMMES . . . . . . . . . . . . . . $\ldots \ldots \ldots \ldots \ldots \ldots$

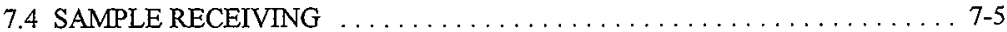

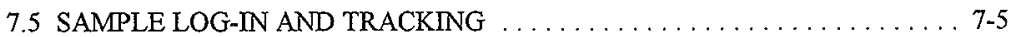

7.5.1 Sample Preservation ........................ 7-5

7.6 LABORATORY INTERNAL CHAIN OF CUSTODY $\ldots \ldots \ldots \ldots \ldots \ldots 7.5$

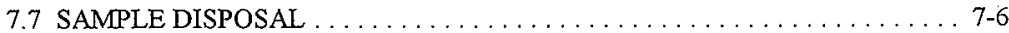

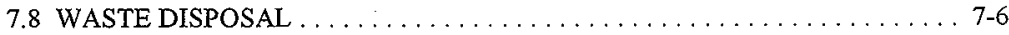

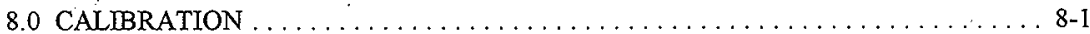

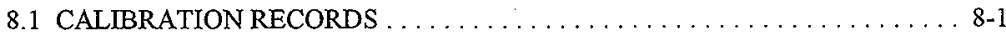

8.2 BALANCES, THERMOMETERS, AND PIPETTES $\ldots \ldots \ldots \ldots \ldots \ldots \ldots$. 1

8.2 .1 Balances . . . . . . . . . . . . . . . . . . . . . . 8-1

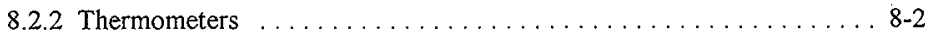

8.2.3 Pipettes, Burets, and Dispensers $\ldots \ldots \ldots \ldots \ldots \ldots \ldots \ldots \ldots .8 .2$

8.3 GENERAL REQUIREMENTS FOR STANDARDS $\ldots \ldots \ldots \ldots \ldots \ldots .8-2$

8.4 CALIBRATION OF LABORATORY MEASUREMENT SYSTEMS (LMS) . . 8-3

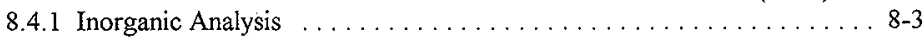

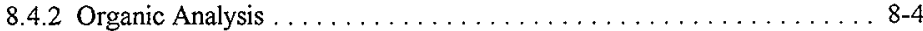

8.4 .3 Physical Testing $\ldots \ldots \ldots \ldots \ldots \ldots \ldots \ldots \ldots \ldots \ldots \ldots \ldots \ldots \ldots \ldots, 4$

9.0 LABORATORY PROCEDURES . . . . . . . . . . . . . . . . . . 9-1

9.1 TECHNICAL AND ADMINISTRATIVE PROCEDURES $\ldots \ldots \ldots \ldots \ldots 9-1$

9.1.1 Preparation and Review of Supporting Documents ........... 9-1

9.2 DOCUMENT CONTROL AND +CHANGE CONTROL . . . . . . . . . . 9-2

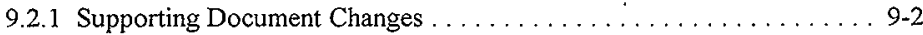

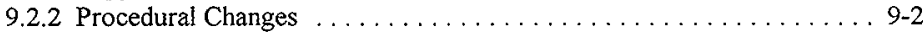

9.2.3 Procedure Cancellation ...................... 9-4

9.3 NEW ANALYTICAL METHODS $\ldots \ldots \ldots \ldots \ldots \ldots \ldots \ldots \ldots . \ldots . \ldots \ldots$

9.4 MODIFICATION OF REQUIRED REGULATORY METHODS $\ldots \ldots \ldots .9-4$

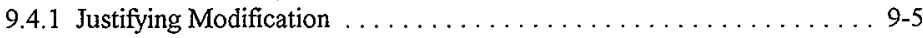

9.4.2 Regulatory Notification ................... $9-5$

9.4.3 Documenting the Modified Method ................ 9-5

9.4.4 Reporting Results from Modified Regulatory Methods .......... . 9-6

9:4.5 Acceptance Criteria for Modified Methods ............... 9-6

9.5 QUALIFICATION OF ANALYTICAL METHODS . . . . . . . . . . . 9.6

10.0 DATA COLLECTION, REDUCTION, AND REPORTING $\ldots \ldots \ldots \ldots \ldots \ldots, 10-1$

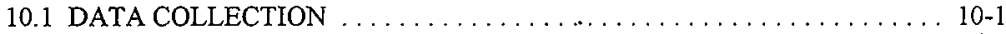




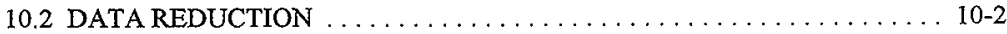

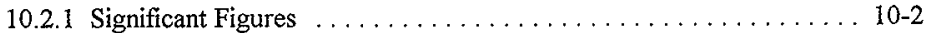

10.2.2 Rounding-Off Methods ..................... 10-3

10.3 DATA REVIEW ............................... 10-4

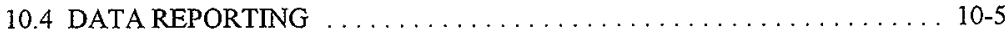

10.4.1 Data Reporting Formats ................... 10-6

10.4.2 Data Reporting Documentation .................. 10-8

10.4.3 Data Package Structure (Generic) . . . . . . . . . . . . 10-12

10.4.4 Preliminary Reporting ...................... 10.13

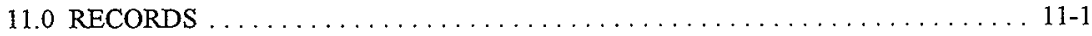

11.1 GENERATION OF QUALITY RECORDS $\ldots \ldots \ldots \ldots \ldots \ldots \ldots \ldots, 11-2$

11.2 RECEIPT CONTROL . . . . . . . . . . . . . . . . . . . . $11-3$

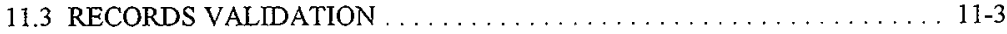

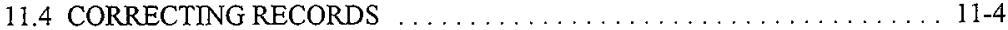

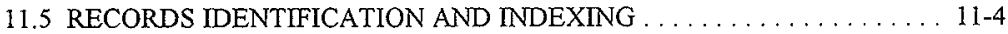

11.6 MAINTENANCE AND RETENTION OF RECORDS . . . . . . . . . . 11-4

11.7 RETRIEVAL AND DISPOSITION ..................... 11-5

11.8 REPLACEMENT, RESTORATION, OR SUBSTITUTION OF RECORDS . 11-5

11.9 RECORDS TURNOVER TO CLIENT . . . . . . . . . . . . . . . 11-5

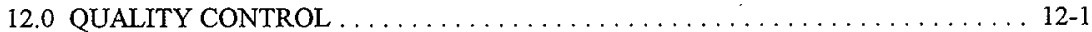

12.1 GENERAL LABORATORY QUALITY CONTROL $\ldots \ldots \ldots \ldots \ldots \ldots .12-2$

12.1.1 Reagent Water . . . . . . . . . . . . . . . . . . . .

12.1.2 Compressed Gases/Reagents .................... 12-2

12.1 .3 Standards . . . . . . . . . . . . . . . . . . . . . . . $12-3$

12.1.4 Labware ................................ 12-3

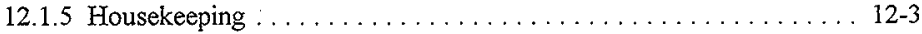

12.2 INORGANIC PREPARATION TECHNIQUES . . . . . . . . . . . 12-3

12.2.1 Laboratory Control Sample or Blank Spike . . . . . . . . . 12-3

12.2.2 Matrix Spike ......................... 12-4

12.2.3 Laboratory Sample Duplicate or Matrix Spike Duplicate . . . . . . . 12-5

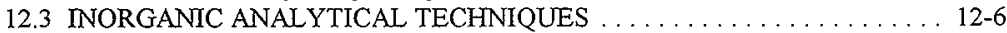

12.3.1 Analytical Run or Sequence . . . . . . . . . . . . . . . 12-7

12.3.2 Initial Calibration Verification . . . . . . . . . . . . . . 12-7

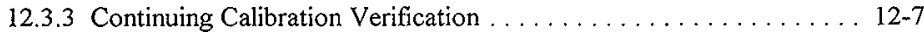

12.3.4 Initial and Continuing Calibration Blanks . . . . . . . . . 12-8

12.3.5 Internal Standards . . . . . . . . . . . . . . . . . . . . $12-8$

12.3.6 Low-Level Standard . . . . . . . . . . . . . . . . . . . 12-8

12.3.7 Interference Check Standards . . . . . . . . . . . . . . . 12-8

12.3 .8 Analytical Spike . . . . . . . . . . . . . . . . . . . . . 12-9

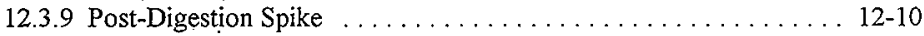

12.3.10 Serial Dilution . . . . . . . . . . . . . . . . . . . 12-10

12.3.11 Method of Standard Additions $\ldots \ldots \ldots \ldots \ldots \ldots \ldots \ldots, 12-11$

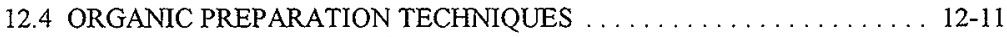

12.4.1 Preparation Batch . . . . . . . . . . . . . . . . . . . 12-11

12.4.2 Preparation Blank (Method Blank) ............... 12-12

12.4.3 Laboratory Control Sample or Blank Spike ............ 12-12 
12.4.4 Matrix Spike . . . . . . . . . . . . . . . . . . 12-13

12.4.5 Laboratory Sample Duplicate or Matrix Spike Duplicate . . . . . . 12-14

12.4.6 Initial Calibration Verification ................... 12-15

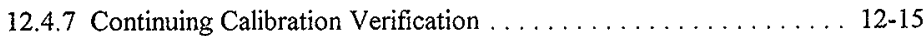

12.4.8 Continuing Calibration Blank $\ldots \ldots \ldots \ldots \ldots \ldots \ldots \ldots \ldots \ldots \ldots \ldots \ldots$

12.4.9 Internal Standards . . . . . . . . . . . . . . . . . . . . . 12-16

12.4.10 Low-Level Standard ..................... 12-17

13.0 PROCEDURES TO ASSESS DATA QUALITY $\ldots \ldots \ldots \ldots \ldots \ldots \ldots .13-1$

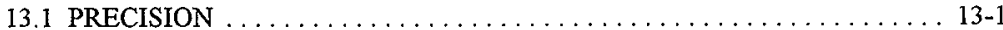

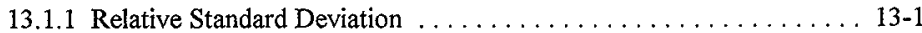

13.1.2 Relative Percent Difference . . . . . . . . . . . . . . . . 13-2

13.2 ACCURACY . . . . . . . . . . . . . . . . . . . . . . 13-2

13.2.1 Method Accuracy Based on Sample Spike ............. 13-2

13.2.2 Method Accuracy Based on Standard ................ 13-2

13.3 MEASURES OF AGREEMENT . . . . . . . . . . . . . . . 13-3

13.3.1 Percent Difference . . . . . . . . . . . . . . . . . . . . . 13-3

13.3.2 Bias ................................. 13-3

13.3.3 Mean Difference ......................... 13-4

13.4 DETECTION LIMIT CONSIDERATIONS $\ldots \ldots \ldots \ldots \ldots \ldots \ldots \ldots . \ldots \ldots$

13.4.1 Inorganic And Organic Methods . . . . . . . . . . . . . 13-4

13.4.2 Limit of Detection . . . . . . . . . . . . . . . . . . 13-8

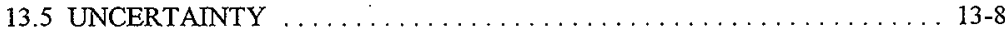

13.6 CONTROL CHARTS . . . . . . . . . . . . . . . . . . . . . . . . . 13-9

13.7 MISCELLANEOUS CALCULATIONS $\ldots \ldots \ldots \ldots \ldots \ldots \ldots \ldots \ldots \ldots$

13.7.1 Response Factor . . . . . . . . . . . . . . . . . 13-10

13.7.2 Percent Difference of the Response Factor $\ldots \ldots \ldots \ldots \ldots \ldots$ 13-10

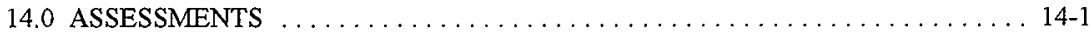

14.1 MANAGEMENT ASSESSMENTS $\ldots \ldots \ldots \ldots \ldots \ldots \ldots \ldots \ldots \ldots . \ldots \ldots, 14$

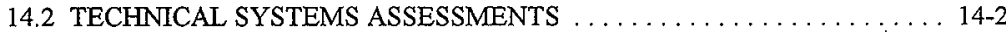

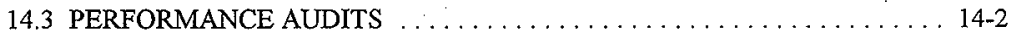

14.4 DATA QUALITY ASSESSMENTSARETE $\ldots \ldots \ldots \ldots \ldots \ldots \ldots$ 14-4

14.5 EXTERNAL AUDITS OR ASSESSMENTS . . . . . . . . . . . . 14-4

15.0 INSTRUMENT PREVENTIVE MANTENANCE $\ldots \ldots \ldots \ldots \ldots \ldots \ldots \ldots$

16.0 CORRECTIVE ACTION AND QUALITY IMPROVEMENT . . . . . . . . . . 16-1

16.1 INITIATION OF CORRECTIVE ACTION ................ 16-1

16.2 EVALUATING IMPACT AND EVENT INVESTIGATION . . . . . . . . 16-2

16.3 ROOT CAUSE ANALYSIS . . . . . . . . . . . . . . . . . . 16-3

16.4 RECURRING CONDITIONS ADVERSE TO QUALITY . . . . . . . 16-3

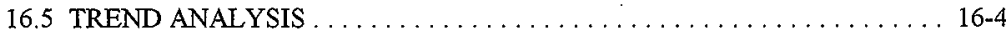

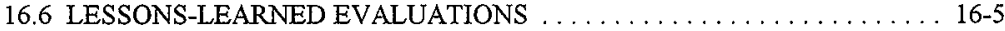

16.7 CONTINUOUS QUALITY IMPROVEMENT $\ldots \ldots \ldots \ldots \ldots \ldots \ldots \ldots .6 \ldots \ldots$

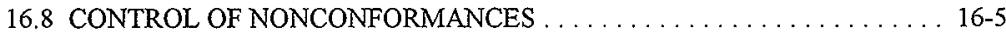

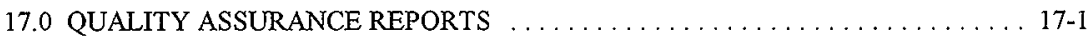


18.0 DATA VALIDATION

19.0 PROCUREMENT CONTROLS . . . . . . . . . . . . . . . . . . . . . . . . . . 19-1

19.1 PURCHASE OF COMMERCIAL CHEMICAL STANDARDS $\ldots \ldots \ldots \ldots$ 19-1

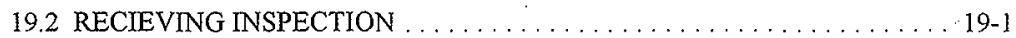

19.3 IDENTIFIED NONCONFORMANCES . . . . . . . . . . . . . 19-1

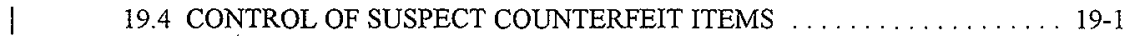

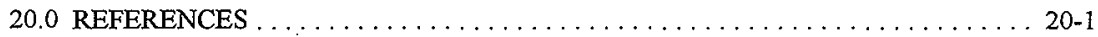
20.1 HANFORD NUCLEAR FACILITY, HANFORD ANALYTICAL LABORATORY

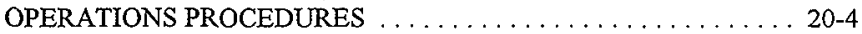
20.2 WASTE MANAGEMENT FEDERAL SERVICES HANFORD. INC., POLICIES/PROCEDURES ................... 20-8 20.3 WESTINGHOUSE HANFORD COMPANY CONTROL MANUALS . . . . 20-9 


\section{LIST OF FIGURES}

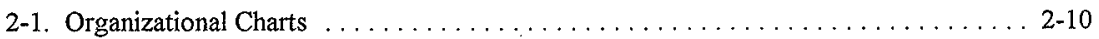

\section{LIST OF TABLES}

1-1. Special Analytical Support QA Cross-reference ................... 1-5

1-2. Special Analytical Support 10CFR 830.120 Cross Reference $\ldots \ldots \ldots \ldots \ldots \ldots \ldots$ 1-8

2-1. SAS Capabilities ................................ 2-13

5-1. Quality Control Level Requirements . . . . . . . . . . . . . . . . . . . . 5-2

$6-1$. Facility Identification . . . . . . . . . . . . . . . . . . . . . . . . . 6-4

7-1. Holding Times for Aqueous Matrices:

Based on SW-846 Regulatory Requirements . . . . . . . . . . . . . 7-7

7-2. Holding Times for Organic Analyses: Based on Regulatory Requirements . . . . . . 7-8

11-1. Recommended Report Deliverables . . . . . . . . . . . . . . . . . . . 11-6

11-2. SAS Level D Quality Control Order of Sample Run . . . . . . . . . . . . . 11-7

15-1. Recommended Preventive Maintenance Frequency for Specific Instruments . . . . . . 15-3

16-1. Recommended Field Laboratory QA/QC Requirements $\ldots \ldots \ldots \ldots \ldots$. . . . . . . . . . 


\section{LIST OF TERMS}

$\% \mathrm{D}$

$\% \mathrm{R}$

AA

ACE

ADP

ALARA

ASTM

BS

CAA

CBRS

CCB

$\mathrm{CCV}$

CERCLA

I

CF

CLP

CM

CWA

DLR

DOE

DOI

DQO

DQR

DTS

DVD

ECN

Ecology

EDT

EPA

EQL

FEB

FDH

FID

FWHM

GC

GC/MS

GEA

GIC

GLP

HALO

HASQARD

percent difference

percent recovery

atomic absorption

Analytical Card Enhancement

Automated Data Processing

as low as reasonably achievable

American Society for Testing Materials

blank spike

Clean Air Act of 1955

Component Based Recall System

continuing calibration blank

continuing calibration verification

Comprehensive Environmental Response, Compensation, and

Liability Act of 1980

calibration factor

Contract Laboratory Program

control manual

Clean Water Act of 1977

decision-level count rate

U.S. Department of Energy

Daily Operating Instructions

data quality objective

data quality requirement

defiency tracking system

data validation deliverable

Engineering Change Notice

Washington State Department of Ecology

engineering data transmittal

U.S. Environmental Protection Agency

estimated quantitation limit

Facility Evaluation Board

Fluor Daniel Hanford, Inc.

Flame Ionization Detector

full-width half maximum

gas chromatograph

gas chromatograph/mass spectrometer

gamma energy analysis

General Inventory Catalog

Good Laboratory Practices

Hanford Analytical Laboratory Operations

Hanford Analytical Services Quality Assurance Requirements Document 


\title{
LIST OF TERMS (Continued)
}

\author{
HATS \\ HLAN \\ $\mathrm{HNF}$ \\ 1 HPLC \\ HPEP \\ IC \\ ICAT \\ ICB \\ ICP \\ ICP/MS \\ ICP/OES \\ ICV \\ IDL \\ ISE \\ JCS \\ LABQAP \\ LAP \\ LC \\ LCS \\ LIMS \\ LLE \\ LMS \\ LMSI \\ LO \\ LOI \\ LQAO \\ LSC \\ LTS \\ MDA \\ MDC \\ MDL \\ MOU \\ MS \\ N/A \\ NA \\ NCR \\ $\mathrm{ND}$ \\ NESHAP \\ NIST \\ NHC \\ Hanford Action Tracking System \\ Hanford Local Area Network \\ Hanford Nuclear Facility \\ High-Performance Liquid Chromatography \\ Hanford Performance Evaluation Program \\ ion chromatography \\ Integrated Contractor Audit Team \\ initial calibration blank \\ inductively coupled plasma (spectrometer) \\ inductively coupled plasma (spectrometer)/mass spectrometer \\ inductively coupled plasma (optical emission spectroscopy) \\ initial calibration verification \\ instrument detection limit \\ Ion Selective Electrodes \\ job control system \\ 222-S Laboratory Quality Assurance Plan \\ Laboratory Administrative Procedure \\ laboratory computer (procedure) \\ laboratory control sample \\ Laboratory Information Management System \\ Lessons Learned Evaluations \\ Laboratory Measurement System \\ Lockheed Martin Services, Inc. \\ laboratory operating (procedure) \\ Letter of Instruction \\ Laboratory Quality Assurance Officer \\ liquid scintillation counter \\ Laboratory Training System \\ minimum detectable activity \\ minimum detectable concentration \\ method detection limit \\ Memorandum of Understanding \\ mass spectrometer \\ not applicable \\ not available \\ non-conformance reports \\ not detectable \\ "National Emission Standards for Hazardous Air Pollutants" \\ National Institute of Standards and Technology \\ Numatec Hanford Corporation
}


HNF-SD-WM-QAPP-034 Rev. 3

\section{LIST OF TERMS (Continued)}

\begin{tabular}{|c|c|}
\hline OJT & on-the-job training \\
\hline O\&RS & Occupational \& Radiological Safety \\
\hline OSHA & Occupational Safety and Health Act of 1970 \\
\hline $\mathrm{PC}$ & project coordinator \\
\hline PCA & Procedure Change Authorization \\
\hline PCB & polychlorinated biphenyl \\
\hline PDP & Performance Demonstration Program \\
\hline PDS & post digestion spike \\
\hline PE & performance evaluation \\
\hline PNNL & Pacific Northwest National Laboratory \\
\hline POD & Plan-Of-The-Day \\
\hline PRAF & procedure review and approval form \\
\hline QA & quality assurance \\
\hline QAPP & Quality Assurance Program Plan \\
\hline QAPP & Quality Assurance Project Plan \\
\hline QC & quality control \\
\hline $\mathrm{QS}$ & Quality Systems \\
\hline RCRA & Resource Conservation and Recovery Act of 1976 \\
\hline RF & response factor \\
\hline RHA-MIS & Records Holding Area - Management Information System \\
\hline RIDS & Records Inventory Disposition Schedule \\
\hline $\mathrm{RL}$ & U.S. Department of Energy, Richland Operations Office \\
\hline RO & reverse osmosis \\
\hline RPD & relative percent difference \\
\hline RRF & relative response factor \\
\hline RSA & request for special analysis \\
\hline RSD & relative standard deviation \\
\hline SAP & Sampling and Analysis Plan \\
\hline SD & supporting document \\
\hline SNM & special nuclear material \\
\hline SOW & statement of work \\
\hline SPC & statistical process control \\
\hline SPCC & system performance check compounds \\
\hline SV & semivolatile (organics) \\
\hline TC & tank coordinator \\
\hline TCLP & toxicity characteristics leaching procedure \\
\hline TMX & Training Matrix Program System \\
\hline TOC & total organic carbon \\
\hline $\begin{array}{l}\text { Tri-Party Agreement } \\
\text { TRU }\end{array}$ & $\begin{array}{l}\text { Hanford Federal Facility Agreement and Consent Order } \\
\text { transuranic }\end{array}$ \\
\hline TSAP & Tank Sample Analysis Plan \\
\hline TSCA & Toxic Substances Control Act 1976 \\
\hline TSD & treatment, storage, and disposal \\
\hline
\end{tabular}




\section{LIST OF TERMS (Continued)}

TWRS

VOA

VTSR

WAC

WHC

WMH

WMFSH

WP

WSCF
Tank Waste Remediation Systems volatile organic analyte (analysis)

validated time of sample receipt

Washington Administrative Code

Westinghouse Hanford Company

Waste Management Hanford

Waste Management Federal Services of Hanford, Inc.

Water Pollutant Studies

Waste Sampling and Characterization Facility 
HNF-SD-WM-QAPP-034 Rev. 3

\subsection{INTRODUCTION}

This quality assurance program plan (QAPP) provides quality assurance (QA) guidance, regulatory QA requirements (for example, Title 10 Code of Federal Regulations (CFR) Part 830.120), and quality control (QC) specifications for services provided by Special Analytical Support (SAS). This document complies with the requirements of the Hanford Analytical Services Quality Assurance Requirements Document (HASQARD) (DOE-RL 1995). The HASQARD is issued and revised by the Waste Management Laboroatory Program Management of the Waste Management Division, U. S. Department of Energy, Richland Operations Office (RL). The HASQARD establishes quality requirements in response to U.S. Department of Energy (DOE) Order 5700.6C (DOE 1991). In addition, this document meets the objectives of the Quality Assurance Program provided in the HNF-MP-599, Project Hanford Quality Assurance Program Description (Beyers and Grier 1997), HNF-SD-WM-QAPP-036, WMFSH Quality Assurance Program Plan (Jacobsen 1998), and HNF-2599, TRU Waste Isolation Pilot

1. Program Quality Assurance. Project Plan (Maupin, 1998). Quality assurance elements required in the QAMS-004, Guidelines and Specifications for Preparing Quality Assurance Program Plans (EPA 1980) from the U.S. Environmental Protection Agency (EPA) are covered throughout this document.

Implementation of the requirements specified in this QAPP results in data necessary to meet a number of objectives. From a programmatic viewpoint, it encompasses primarily the characterization of wastes at the Hanford Site. From a regulatory compliance viewpoint, the QAPP addresses several data needs associated with regulatory compliance programs. The QAPP defines the sampling and analytical techniques requirements to support regulatory compliance programs for the Hanford Site.

This QAPP will be reviewed annually, and revised as necessary. The data quality objectives (DQO) and associated information needs will be revised as needed in response to data users. This conforms to the assertion found in Guidance for the Data Quality Objectives Process, (EPAQAG/G-4) (EPA 1994E), that the DQO process is iterative in nature and can be used repeatedly throughout projects. During the annual review of the QAPP, the DQO process will be used to ensure that the QAPP remains current with respect to the needs of the end users of data generated from SAS activities.

Table 1-1 is a QA cross reference or matrix reflecting 10 CFR 830.120, "Quality Assurance Criteria." Table 1-2 provides a matrix of HASQARD requirements and SAS procedures and policies used to implement these requirements.

This document also provides and/or identifies the procedural information that governs laboratory operations. The personnel of SAS including managers, analysts, QA/QC staff, and support staff shall use this document as guidance and instruction for their operational quality assurance activities. Other organizations that conduct activities described in this document for SAS shall follow this QA/QC document. 
A graded approach will be tutitizedused for all quality-affecting activities performed by SAS. The graded approach takes into account such factors as:

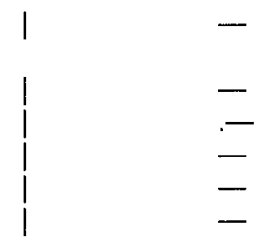

Level of risk to workers, facilities, and the environment, and impact associated with a failure or deficiency: Age, status, and condition of a facility; or process; or an itemHistory of problems at a facility; with a process, method, or item: Current safety documentation:

Complexity of products or activities involved:

Control of potential project delays and costs; if failures or deficiencies occur.

All sampling and analysis performed by SAS shall meet or exceed the QA/QC protocols described in this QAPP. This QAPP addresses the relevant quality aspects of the SAS laboratory operation applicable to sampling and analytical protocols that apply to environmental samples collected to meet requirements of the Resource Conservation and Recovery Act of 1976 (RCRA) and the Comprehensive Environmental Response, Compensation, and Recovery Act of 1980 (CERCLA), analysis of air, drinking water, groundwater, surface water, waste water, soil, sludge, sediment, solid and hazardous waste, waste tank headspace vapors, toxic substances, and other matrices. Examples include, but are not limited to, waste characterization for the Tank Waste Remediation Systems (TWRS), waste characterization for regulatory waste treatment, storage, and disposal (TSD), regulatory compliance samples, radiation screening, process samples, evaporator samples, effluent samples), and samples collected to meet the requirements of the Hanford Federal Facility Agreement and Consent Order (Tri-Party Agreement) (Ecology et al. 1996). A graded approach is applied on the level of sample custody, QC, data verification, and data reporting to meet the specific needs of the client.

The QA/QC requirements of this document will be used for all sampling and analytical work except when modified as less or more restrictive by the customer or project-specific or data quality requirements (DQRs) and communicated to SAS in the project-specific quality assurance project plan (QAPjP), sampling and analysis plan or other work-authorizing documents.

Customer requirements will be documented for most cases in the case narrative. Communication with the customer shall clearly specify the actions required should the QC criteria fail (that is, rerun, flag in narrative, or qualify on report summary). Alternative methods of sample analysis, data reporting, and data validation may be used to meet work-plan DQOs with approval and documentation by the project lead and/or customer.

\subsection{ACCREDITATIONS}

SAS maintains accreditation with the Washington Department of Ecology (Ecology) through the analysis of water pollutant study samples and audits by Ecology. SAS also is applying for accreditation with the Washington Department of Health and currently is involved in becoming a Certified Laboratory for the Waste Isolation Pilot Program (WIPP), which involves demonstrating proficiency of analyses through the Performance Demonstration Program and an onsite audit by 
the WIPP project. SAS continues to be proactive in seeking additional accreditations, such as ISO-9001, and will continue researching other possibilities.

\subsection{MISSION STATEMENT}

The SAS management and staff are committed to establishing and maintaining a rigorous QA program for all aspects of laboratory and field operation. SAS is to establish "Best in Class" for the field assessment services, in situ measurements, and organic sampling and analysis to build a team of experts to provide scientific and technical leadership, thereby supporting the Hanford Site's monitoring, retrieval, remediation, and restoration projects. The goal is to perform chemical screening and analysis of water, soil, waste, and vapor samples from environmental sampling programs conducted on the Hanford Site in a timely manner in accordance with the DQO.

The group supports the mission by providing rapid turnaround sampling and analysis services. SAS promotes field analysis by the following:

- Field deployable analysis and screening services;

- Expert support in developing or modifying project analytical requirements;

- Rapid-turnaround, low-cost, protocol-based analyses in support of waste designation and site characterization activities, and,

- Regulatory analytical supp.

The primary scope of work within SAS is establishing the required organic sampling and analytical systems. These capabilities are supported by mobile and fixed laboratory facilities to provide onsite and field real-time support services for Hanford Site projects. All the sampling and analytical activities will comply with the site standard Quality Assurance Program (QAP),

HASQARD or other programs' specific QAPJPs. SAS provides this type of support in the following ways;

- Development, review, and implementation of DQO-based vapor sampling and analysis plans on site and throughout the Hanford site.

- Turn-key, custom-tailored emissions, atmospheric, and headspace monitoring services;

- Screening and field analysis to monitor emissions during retrieval, remediation, and restoration project activities.

- Comprehensive tank vapor sampling and analysis support. 
HNF-SD-WM-QAPP-034 Rev، 3

This page intentionally left blank. 
HNF-SD-WM-QAPP-034 Rev. 3

Table 1-1. Special Analytical Support QA Cross-reference. (3 sheets)

\begin{tabular}{|c|c|c|}
\hline $\begin{array}{c}\text { SAS QA Plan } \\
\text { HINF-SD-WM-QAPP-034 by Section }\end{array}$ & $\begin{array}{l}\text { HASQARD } \\
\text { DOE/RL-96-68 }\end{array}$ & $\begin{array}{l}\text { Procedures and } \\
\text { Pólicies }\end{array}$ \\
\hline 1.0 INTRODUCTION & Volume 1, Section. 1.0 & $\begin{array}{l}\text { HNF-SD-WM-QAPP-036, Sec. 3.1.1, } \\
\text { HNF-MP-599, WHC-SP-1131 }\end{array}$ \\
\hline $\begin{array}{l}\text { 2.0 ORGANIZATION AND } \\
\text { RESPONSIBLITY }\end{array}$ & Volume 1 , Section 2.0 & HNF-PRO-261, WMH-100, Sec. 4 , \\
\hline $\begin{array}{l}\text { 3.0 PERSONNEL QUALIFICATION AND } \\
\text { TRAINING }\end{array}$ & Volume 1, Section 3.0 & $\begin{array}{l}\text { WMH-200, Sec. } 5.1 \\
\text { HNF-PRO-167 through - } 179 \\
\text { HNF-MP-599, P2, Sec. } 2 \\
\text { HNF-3275 }\end{array}$ \\
\hline $\begin{array}{l}\text { 4.0 QUALITY ASSURANCE OBJECTIVES } \\
\text { 4.1 Data Quality Objectives } \\
\text { 4.2 Client Data Quality Requirements }\end{array}$ & $\begin{array}{l}\text { Volume 4, Section } 1.0 \\
\text { Volume 4, Section } 1.1 \\
\text { Volume 4, Section } 1.2\end{array}$ & $\begin{array}{l}\text { Client's QAPjP, or work authorization } \\
\text { document }\end{array}$ \\
\hline $\begin{array}{l}\text { 5.0 FIELD ANALYTICAL SUPPORT } \\
\text { LEVELS }\end{array}$ & & 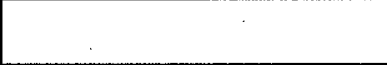 \\
\hline $\begin{array}{l}\text { 6.0 SYSTEMS QUALITY ASSURANCE } \\
\text { 6.1 Software Systems } \\
6.2 \text { Technical/Administrative Systems } \\
6.3 \text { Physical Facilities Systems } \\
\end{array}$ & $\begin{array}{l}\text { Volume 4, Section } 2.0 \\
\text { Volume 1, Section } 6.0 \\
\text { Volume 4, Section } 2.1 \\
\text { Volume 4, Section } 2.2 \\
\end{array}$ & $\begin{array}{l}\text { HNF-PRO-309, HNF-SD-WM-CM-002', } \\
\text { HNF-PRO-433 } \\
\text { LC-100-400 } \\
\text { HNF-SD-CP-ISB-002 }\end{array}$ \\
\hline $\begin{array}{l}\text { 7.0 CUSTODY AND HANDLING } \\
\text { 7.1 Chain of Custody } \\
\text { 7.2 Holding Times } \\
\text { 7.3 Sample Receiving Procedure } \\
\text { 7.4 Sample Log-in and Tracking Procedure } \\
\text { 7.5 Laboratory Internal Chain-of-Custody } \\
\text { 7.6 Sample Disposal } \\
\text { 7.7 Waste Disposal }\end{array}$ & $\begin{array}{l}\text { Volume 4, Section } 3.0 \\
\text { Volume 4, Section } 3.1 \\
\text { Volume 4, Section } 3.2 \\
\text { Volume 4, Section } 3.3 \\
\text { Volume 4, Section } 3.4 \\
\text { Volume 4, Section } 3.5 \\
\text { Volume 4, Section } 3.6\end{array}$ & $\begin{array}{l}\text { WMH-200, Sec. } 3.12 \\
\text { LO-090-450, WMH-310-1.4, } \\
\text { WMH-310, Sec. } 2.13 \\
\text { LO-090-405 } \\
\text { LO-090-405 } \\
\text { LO-090-405 } \\
\text { LO-090-405, WMH-310, Sec. } 2.13 \\
\text { LQ-549-400 } \\
\text { LO-100-400, HNE-PRO-451, }-453 \text {, } \\
-455,-459,-462 \text { and }-690\end{array}$ \\
\hline $\begin{array}{l}\text { 8.0 CALIBRATION } \\
\text { 8.1 Calibration Records } \\
\text { 8.2 Balances, Thermometers, and Pipettes } \\
\text { 8.3 General Requirements for Standards }\end{array}$ & $\begin{array}{l}\text { Volume 4, Section } 4.0 \\
\text { Volume 4, Section } 4.1 \\
\text { Volume 4, Section } 4.2 \\
\text { Volume 4, Section } 4.3\end{array}$ & $\begin{array}{l}\text { Specific Analytical Procedure } \\
\text { As Per Method or Resp. Chemist } \\
\text { HNN-IP-1 128, Sec. } 2.10 \\
\text { HNF-IP-1128, Sec. } 1.7,2.14,2.15,2.3 \\
\text { HNF-IP-1128,2.13, WMH-310 Sec. } 1.7\end{array}$ \\
\hline $\begin{array}{l}\text { 9.0 LABORATORY PROCEDURES } \\
\text { 9.1 Procedures and Supporting Documents } \\
\text { 9.2 Change Control } \\
\text { 9.3 New Analytical Methods } \\
\text { 9.4 Modification of Required Regulatory } \\
\text { Methods } \\
\text { 9.5 Qualification of A'nalytical Methods }\end{array}$ & $\begin{array}{l}\text { Volume 4, Section } 5.0 \\
\text { Volume 4, Section } 5.5 \\
\text { Volume 4, Section } 5.3 \\
\text { Volume 4, Section } 5.7 \\
\text { Volume 4, Section } 5.6\end{array}$ & $\begin{array}{l}\text { WMH-310, Sec. } 1.8 \\
\text { HNF-MP-599, P2, Sec. } 5 \\
\text { HNF-IP-1128, 1.0 } \\
\text { WMH-310, Sec. } 1.8,1.11 \text {, } \\
\text { HNF-PRO-440 } \\
\text { WMH-200, Sec. } 1.1 \\
\text { WMH-200, Sec. } 2.16\end{array}$ \\
\hline
\end{tabular}


Table 1-1. Special Analytical Support QA Cross-reference. (3 sheets)

\begin{tabular}{|c|c|c|}
\hline $\begin{array}{c}\text { SAS QA Plan } \\
\text { HNF-SD-WM-QAPP-034 by Section }\end{array}$ & $\begin{array}{c}\text { HASQARD } \\
\text { DOE/RL-96-68 }\end{array}$ & $\begin{array}{c}\text { Procedures and } \\
\text { Policies } \\
\end{array}$ \\
\hline $\begin{array}{l}\text { 10.0 DATA COLLECTION, REDUCTION, } \\
\text { AND REPORTING } \\
\text { 10.1 Data Collection } \\
\text { 10.2 Data Reduction } \\
\text { 10.3 Data Review } \\
\text { 10.4 Data Reporting }\end{array}$ & $\begin{array}{l}\text { Volume } 4, \text { Section } 6.0 \\
\text { Volume 4, Section } 6.1 \\
\text { Volume 4, Section } 6.2 \\
\text { Volume 4, Sec. } 6.2 .3 \\
\text { Volume 4, Section } 6.3\end{array}$ & $\begin{array}{l}\text { HNF-PRO-222 } \\
\text { WMH-310, Sec. } 2.8 \\
\text { WMH-310, Sec. } 2.8\end{array}$ \\
\hline 11.0 RECORDS & Volume 1 , Section 5.0 & $\begin{array}{l}\text { HNF-MP-599 } \\
\text { WMH-200, Sec. 2.1, WMH-310, Sec. } 1.3 \\
\text { HNF-PRO-208, -210 through }-216 \text {, and } \\
-222 \text { through }-233\end{array}$ \\
\hline $\begin{array}{l}\text { 12.0 QUALITY CONTROL } \\
\text { 12.1 General Laboratory Quality Control } \\
\text { 12.4 Inorganic Preparative Techniques } \\
\text { 12.5 Inorganic Analytical Techniques } \\
\text { 12.6 Organic Preparative Techniques } \\
\text { 12.7 Organic Analytical Techniques }\end{array}$ & $\begin{array}{l}\text { Volume 4, Section } 7.0 \\
\text { Volume 4, Section } 7.1 \\
\\
\text { Volume 4, Section } 7.3 \\
\text { Volume 4, Section } 7.4 \\
\text { Volume 4, Section 7.5 } \\
\text { Volume 4, Section 7.6 } \\
\text { Volume 4, Section } 7.7 \\
\text { Volume 4, Section } 7.8\end{array}$ & $\begin{array}{l}\text { Specific LA procedures } \\
\text { Customer TSAP or work authorization } \\
\text { document } \\
\text { WMH-3 10, Sec. } 8.5 \\
\text { HNF-IP-1 128, Sec. } 1.2 \\
\text { HNF-IP-1128, Sec. } 2.5 \\
\text { Standing Order 96-003 } \\
\text { Standing Order } 96-003 \\
\text { Standing Order } 96-003\end{array}$ \\
\hline $\begin{array}{l}\text { 13.0 PROCEDURES TO ASSESS DATA } \\
\text { QUALITY } \\
\text { 13.1 Precision } \\
\text { 13.2 Accuracy } \\
\text { 13.4 Measures of Agreement } \\
\text { 13.5 Detection Limit Considerations } \\
\text { 13.6 Uncertainty } \\
\text { 13.7 Control Charts } \\
\text { 13.8 Miscellaneous Calculations }\end{array}$ & $\begin{array}{l}\text { Volume 4, Section } 8.0 \\
\text { Volume 4, Section } 8.2 \\
\text { Volume 4, Section } 8.3 \\
\text { Volume 4, Section } 8.5 \\
\text { Volume 4, Section } 8.6 \\
\text { Volume 4, Section } 8.7 \\
\text { Volume 4, Section } 8.8 \\
\text { Volume 4, Section } 8.1\end{array}$ & $\begin{array}{l}\text { Specific LA procedures } \\
\text { HNF-IP-1 } 128, \text { Sec. } 2.11 \\
\text { HNF-IP-1 } 128, \text { Sec. } 2.6\end{array}$ \\
\hline $\begin{array}{l}\text { 14.0 AUDITS/ASSESSMENTS } \\
\text { 14.1 Management Assessments } \\
\text { 14.2 Technical Systems Audits } \\
\text { 14.3 Performance Audits } \\
\text {. } \\
\text { 14.4 Data Quality Audits } \\
\text { 14.5 External Audits/Assessments }\end{array}$ & $\begin{array}{l}\text { Volume 1, Section } 9.0 \\
\text { Volume 1, Section } 9.1 \\
\text { Volume 1, Section } 9.2 \\
\text { Volume 1, Section } 9.3 \\
\\
\text { Volume 1, Section } 9.4 \\
\text { Volume 1, Section } 9.5\end{array}$ & $\begin{array}{l}\text { HNN-MP-599 } \\
\text { HNF-PRO-246, WMH-200, Sec. } 1.2 \\
\text { WHC-SD-QAPP-002 } \\
\text { HNE-PRO-439 } \\
\text { HNF-MP-599, P2, Sec. } 10\end{array}$ \\
\hline $\begin{array}{l}\text { 15.0 INSTRUMENT PREVENTIVE } \\
\text { MAINTENANCE }\end{array}$ & Volume 1, Section 8.0 & $\begin{array}{l}\text { As per method } \\
\text { WMH-310, Sec } 3.7\end{array}$ \\
\hline
\end{tabular}


HNF-SD-WM-QAPP-034 Rev. 3

Table 1-1. Special Analytical Support QA Cross-reference. (3 sheets)

\begin{tabular}{|c|c|c|}
\hline $\begin{array}{c}\text { SAS QA Plan } \\
\text { HNF-SD-WM-QAPP-034 by Section }\end{array}$ & $\begin{array}{c}\text { HASQARD } \\
\text { DOE/RL-96-68 }\end{array}$ & $\begin{array}{l}\text { Procedures and } \\
\text { Policies }\end{array}$ \\
\hline $\begin{array}{l}\text { 16.0 CORRECTIVE ACTION AND } \\
\text { QUALITY IMPROVEMENT } \\
\text { 16.1 Initiation of Corrective Action } \\
\text { 16.2 Evaluating Impact and Event Investigation } \\
\text { 16.3 Root Cause Analysis } \\
\text { 16.4 Recurring Conditions Adverse to Quality } \\
\text { 16.5 Trend Analysis } \\
\text { 16.6 Lessons Learned Evaluations } \\
\text { 16.7 Continuous Quality Improvement } \\
\text { 16.8 Control of Nonconformances }\end{array}$ & $\begin{array}{l}\text { Volume 1, Section } 4.0 \\
\text { Volume 1, Section } 4.1 \\
\text { Volume 1, Section } 4.2 \\
\text { Volume 1, Section } 4.3 \\
\text { Volume 1, Section } 4.4 \\
\text { Volume 1, Section } 4.5 \\
\\
\text { Volume 1, Section } 4.6 \\
\text { Volume 1, Section } 4.7\end{array}$ & $\begin{array}{l}\text { HNF-MP-599, WMH-200 Sec. } 1.3, \\
\text { HNF-PRO-052 } \\
\text { WMH-310 Sec. } 8.10 \\
\text { WMH-310 Sec. } 8.10 \\
\text { HNF-PRO-052 } \\
\text { HNF-IP-1128, } 2.6 \\
\text { HNF-200 Sec. 1.11, HNF-PRO-067 } \\
\text { 10 CFR } 830.120, \text { WHC-SP-1131 C3 } \\
\text { HNF-MP-599, and HNF-PRO-298 }\end{array}$ \\
\hline 17.0 QUALITY ASSURANCE REPORTING & Volume 1 , Section 10.0 & LQ-529-400 \\
\hline 18.0 DATA VALIDATION & Volume 4 , Section 9.0 & N/A \\
\hline 19.0 PROCUREMENT CONTROLS & Volume 1 , Section 7.0 & $\begin{array}{l}\text { HNF-SD-WM-QAPP-036 } \\
\text { HNF-MP-599, HNF-PRO-301 } \\
\text { HNF-PRO-238, HNF-PRO-268 }\end{array}$ \\
\hline 20.0 REFERENCES & Volume 1, Sec. 11,0 & N/A \\
\hline
\end{tabular}

Notes: 'Rich, H. S., 1995, Configuration Management Plan for LABCORE Program, HNF-SD-WM-CM-002, Westinghouse Hanford Company, Richland, Washington.
$\mathrm{CM}=$ control manual
LA = laboratory analytical procedure
LC = Iaboratory computer (procedures)
LO = laboratory operating procedure
$\mathrm{LQ}=$ = laboratory quality control procedure
MOU = memorandum of understanding
$\mathrm{N} / \mathrm{A} \quad=$ not applicable
$\mathrm{QAP}_{\mathrm{j}} \mathrm{P}=$ Quality Assurance Project Plan
SOW $=$ Statement of Work 


\begin{tabular}{|c|c|}
\hline 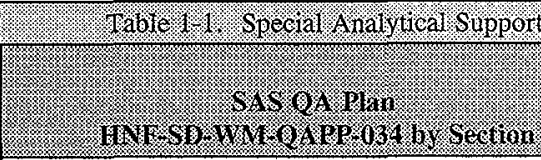 & 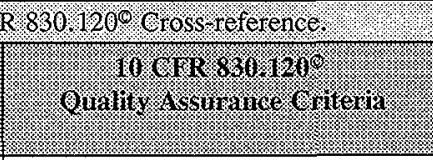 \\
\hline \multicolumn{2}{|l|}{ 1.0 INTRODUCTION } \\
\hline $\begin{array}{l}\text { 2.0 ORGANIZATION AND } \\
\text { RESPONSIBILITY }\end{array}$ & $\begin{array}{l}\text { Quality Assurance Program and } \\
\text { Organization (Program) }\end{array}$ \\
\hline $\begin{array}{l}\text { 3.0 PERSONNEL QUALIFICATION AND } \\
\text { TRAINING } \\
\end{array}$ & Personnel Qualification and Training \\
\hline $\begin{array}{l}\text { 4.0 QUALITY ASSURANCE OBJECTIVES } \\
\text { 4.1 Data Quality Objectives } \\
\text { 4.2 Client Data Quality Requirements }\end{array}$ & $\begin{array}{l}\text { Design Control } \\
\text { Planning Scientific Investigations } \\
\text { Design }\end{array}$ \\
\hline $\begin{array}{l}\text { 5.0 SYSTEMS QUALITY ASSURANCE } \\
\text { 5.1 Software Systems } \\
\text { 5.2 Technical/Administrative Systems } \\
\text { 5.3 Physical Facilities Systems }\end{array}$ & $\begin{array}{l}\text { Work Processes } \\
\text { Software QA Requirements }\end{array}$ \\
\hline $\begin{array}{l}\text { 6.0 CUSTODY AND HANDLING } \\
\text { 6.1 Chain of Custody } \\
\text { 6.2 Holding Times } \\
\text { 6.3 Sample Receiving Procedure } \\
\text { 6.4 Sample Log-in and Tracking Procedure } \\
\text { 6.5 Laboratory Internal Chain-of-Custody } \\
\text { 6.6 Sample Disposal } \\
\text { 6.7 Waste Disposal } \\
\end{array}$ & $\begin{array}{l}\text { Sample Control } \\
\text { Sample Identification } \\
\text { Handling, Storing, Shipping Samples } \\
\text { Disposition of Nonconforming } \\
\text { Samples } \\
\text { Work Processes }\end{array}$ \\
\hline $\begin{array}{l}\text { 7.0 CALIBRATION } \\
\text { 7.1 Calibration Records } \\
\text { 7.2 Balances, Thermometers, and Pipettes } \\
\text { 7.3 General Requirements for Standards }\end{array}$ & $\begin{array}{l}\text { Work Processes } \\
\text { Inspection and Testing } \\
\text { (Work Processes, Inspection, and } \\
\text { Acceptance }\end{array}$ \\
\hline $\begin{array}{l}\text { 8.0 LABORATORY PROCEDURES } \\
\text { 8.1 Procedures and Supporting Documents } \\
\text { 8.2 Change Control } \\
\text { 8.3 New Analytical Methods } \\
\text { 8.4 Modification of Required Regulatory } \\
\text { Methods } \\
\text { 8.5 Qualification of Analytical Methods }\end{array}$ & $\begin{array}{l}\text { Records } \\
\text { Data Documentation, Control and } \\
\text { Qualification } \\
\text { Documents and Records } \\
\text { Work Processes } \\
\text { Performing Scientific Investigation }\end{array}$ \\
\hline $\begin{array}{l}\text { 9.0 DATA COLLECTION, REDUCTION, } \\
\text { AND REPORTING } \\
\text { 9.1 Data Collection } \\
\text { 9.2 Data Reduction } \\
\text { 9.3 Data Review } \\
\text { 9.4 Data Reporting }\end{array}$ & $\begin{array}{l}\text { Records } \\
\text { Data Documentation, Control and } \\
\text { Qualification } \\
\text { cuments and Records }\end{array}$ \\
\hline
\end{tabular}


Table 1 1. Special Analytical Support 10 CER 8301200 Cross reference:

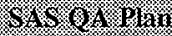

$10 . \mathrm{CrR} 8.30 .120 \%$

Ginali, Assurance Criteria

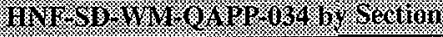

\begin{tabular}{|l|l|}
\hline 10.0 RECORDS & Dociments and Records \\
\hline 11.0 QUALITY CONTROL & Work Processes \\
11.2 Preparat Laboratory Quality Control & Inspection and Testing \\
f/Radiochemistry & Work Processes, Inspection and \\
11.3 Radiochemical Techniques & Acceptance \\
11.4 Inorganic Preparative Techniques & \\
11.5 Inorganic Analytical Techniques & \\
11.6 Organic Preparative Techniques & \\
11.7 Organic Analytical Techniques & \\
11.8 Physical Testing & \\
\hline 12.0 PROCEDURES TO ASSESS DATA & Quata Documentation, Control and \\
12.1 Precision & Qualification \\
12.2 Accuracy & Work Processes \\
12.3 Yield Recovery (Radiochemistry Only) & Design Control \\
12.4 Measures of Agreement & \\
12.5 Detection Limit Considerations & \\
12.6 Uncertainty & \\
12.7 Control Charts & \\
12.8 Miscellaneous Calculations & \\
\hline 13.0 AUDITS/ASSESSMENTS & Quality Improvement \\
13.1 Management Assessments & Management Assessment \\
13.2 Technical Systems Audits & Independent Assessment \\
13.3 Performance Audits & \\
13.4 Data Quality Audits & \\
13.5 External Audits/Assessments & Work Processes \\
\hline 14.0 INSTRUMENT PREVENTIVE & \\
MAINTENANCE & \\
\hline
\end{tabular}




\subsection{ORGANIZATION AND RESPONSIBILITY}

\subsection{SAS MANAGEMENT QUALITY POLICY}

The policy of Fluor Daniel Hanford, Inc. (FDH), Waste Management Federal Services of Hanford, Inc. (WMFSH), and Numatec Hanford Corporation (NHC) Special Analytical Support (SAS) management is to direct activities in a manner that ensures the results meet or exceed the customer's requirements and provides supporting documentation.

It is the policy of Special Analytical Support (SAS) that the analytical aspects of all environmental data generated and processed in the laboratory, subject to the EPA, DOE, Ecology, or other project-specific requirements, be of known and acceptable quality. It is the intention of this QAPP to establish and ensure that an effective quality controlled management system is maintained to meet the quality requirements of the intended use of the data. We will continually strive to understand customer requirements, perform services; and activities that meet or exceed customer expectations, and be cost effective in our performance.

\subsection{SAS ETHICS POLICY}

SAS is committed to maintaining high ethical standards. Quality, health and safety, and regulatory compliance must come before profits. Our quality ethic and quality principles constantly guide our actions. We will meet our own quality expectations and exceed those of our customers with vigilance, commitment, and persistence.

As a member of the PHMC Team, SAS, adheres to FDH policies and procedures. Additionally, SAS applies the policies and procedures of the major subcontractors (WMH, NHC, FDH, Lockheed Martin Hanford Company [LMHC]] to which it is providing management or other services. Figures 2-1 through 2-4 provide an organizational breakdown of the SAS interface with

Technical Direction and Laboratories (TD\&L), $f$ NHC, the Waste Management Laboratory

( $\mathrm{WML}$ ), $\mathrm{WMH}$, and FDH. 


\subsection{ORGANIZATIONAL RESPONSIBILITIES}

SAS reports to NHC. The SAS organization is responsible for providing support in the areas of environmental sampling, field screening $f$ and analysis, vapor sampling, and analytical services The SAS organization is composed of personnel specializing in field analytical sampling, analysis and development, and vapor sampling and headspace gas analysis. Table 2-1 lists SAS's analytical method capabilities.

\subsubsection{Management Responsibilities}

Management is directly responsible for establishing the scope of, and implementing, assessing, and continually improving an effective quality system. Management is-also is responsible in leading, directing, and motivating personnel to correctly perform their assigned duties, improve performance and process, and implement quality to the highest level possible in all aspects of their work.

Management's commitment to establishing an effective quality program shall be implemented through the following:

- All levels of management accept responsibility for their organization's activities and are held accountable for achieving quality.

- Management provides adequate resources and budget to support effective QA practices that fulfill the customer's program goals and performance objectives.

- Management provides facilities, instruments, support equipment, and materials needed to meet current project requirements.

- Management responsibilities include, but are not limited to, the following;

- Managing and operating SAS facilities

- Providing individuals with the information, tools, support, training, and encouragement needed to perform their assigned work

- Assigning personnel who are responsible for ensuring that the operation of the facility and waste management activities are in compliance with federal, state, and local government regulations

- Identifying and controlling hardware and software

- Assigning personnel who are responsible for performing maintenance, repair, and improvements 
- Providing resources and staff necessary to implement the requirements of this QAPP into environmental sampling and testing activities

- Demonstrating commitment and leadership to achieve and consistently improve quality through active involvement and implementation of this QAPP.

Management will let their expectations be known by frequent open and free communications at all levels. Employees are encouraged to bring safety issues and solutions to management attention. All personnel should be held accountable for performance and all shall be treated fairly and with respect. Sufficient staff, equipment, and funding shall be allocated to permit the laboratory to perform its functions. Performance will be closely monitored by facility management.

\subsubsection{All SAS Personnel}

Free and open communication between workers and management is encouraged.

Each SAS staff member has the customer, the person depending on the staff member to do his/her job in a quality way. SAS staff members will work together to make each other's jobs easier, and to succeed as a team. The SAS staff members do this with respect and dignity toward each other.

All personnel are responsible for compliance with the provisions of all applicable regulations and procedures. All personnel shall be responsible for the quality of their work. All personnel are responsible for initiating action to prevent reporting laboratory results from a measurement system that is out of control; prevent further reporting of measurements until corrective action has been completed, and or identify any method or procedure that poses quality problems. Managers and supervisors shall ensure that all personnel under their direction are trained in regard to the conduct of operations.

Workers and their supervisors shall be held accountable for operating performance. Personnel | who frequently violate operating practices shall be counseled, retrained, and disciplined as appropriate.

SAS personnel are responsible for stopping activities when conditions that jeopardize safety or quality are identified. All personnel have authority to stop work they determine represents an imminent hazard and places their personal safety or the safety of their coworkers at risk.

2.3.2.1 Team Leads/Technical Representatives. The team leads and technical representatives have the following responsibilities:

- Ensure that work performance meets the requirements of work plans

- Act as technical advisors to the laboratory 
- Serve as the interface between SAS and customers

- Document and track client complaints and/or issues from receipt through resolution; place all subsequent information in the appropriate QA file

- Organize and plan their team's activities and establish work priorities

- Perform technical reviews of all the reports issued by their team

- Provides technical assistance and guidance to SAS teams for sampling, analysis, data review, and technical data evaluation

- Participates in the review and disposition of nonconformance reports and/or Quality Letters of Observation

- Participates in the development of appropriate SAS quality requirements and this QAPP.

2.3.2.2 Quality Assurance Officer. The quality assurance officer has the following responsibilities:

- Manages document control, including administration of procedures, laboratory certifications, and maintenance of records

- Trains personnel in QA techniques and development of mechanisms to carry out QA/QC objectives

- $\quad$ Administers QA/QC performance testing programs, such as round robins,Performance Demonstration Program (PDP) and Water Pollutant Studies (WP), to ensure QA/QC compliance

- Trains personnel to the applicable requirements in this QAPP, as well as those in the HASQARD

- Documents analyst proficiency testing

- Performs technical self-assessments.

- Assembles and interprets internal control charts, establishes quality indicators, charts internal trend analysis

- $\quad$ Performs QA and QC reviews for all reports issued by SAS

- Reports to management significant accomplishments and recommendations to update or change this QAPP 
- Overseeing the QA program in SAS

- Reviewing analytical laboratory personnel training and qualifications

- Periodically monitoring analytical instrument performance

- Evaluating and verifying data quality through spot-checking

- Periodically reviewing and summarizing $Q C$ reports

- Performing audits/assessment and recommending corrective actions

- Coordinating external auditing between laboratory personnel and external agencies for overall systemic operations

- Provides copies of reports, the internal assessment, assessment schedules, and performance evaluation results to management.

The Quality Assurance Officer may order a suspension of activities if conditions affecting quality have not been addressed by cognizant management.

2.3.2.3 Chemists. The chemists have the following responsibilities:

- $\quad$ Make recommendations to SAS team leads

- Develop methods for screening and/or analyzing materials and/or wastes in a variety of matrices in coordination with SAS team leads.

- Update the analytical procedures they run when needed

- Perform calibrations, sample preparations, and analyses

- Calculate, interpret, and review data as they are generated to ensure optimization. and accuracy of analytical instrumentation

- Perform QA/QC analyses

- Properly record information into controlled laboratory notebooks and/or other media (such as electronic media) depending on customer's requirements

- Maintain strict adherence to approved procedures, this QAPP, and relevant QAPjP requirements

- $\quad$ Provide information to the Quality Assurance Officer as needed

- Pass proficiency testing before analyzing samples 
- Prepare data reports as directed by the team leads or provide the individual in charge of preparing the reports with the relevant technical data and narrative sections

- Perform daily and routine instrument maintenance

2.3.2.4 Analysts and Aids. Analysts and aids have the following responsibilities.

- Perform duties as sample custodian

- Perform sample preparations and analyses

- Follow laboratory procedures for any analytical, glassware cleaning, maintenance, activities, and maintain a clean and safe working environment.-

- Make recommendations to chemists or SAS team leads regarding sample preparations and laboratory cleaning.

- Properly record information into controlled laboratory notebooks.

- Maintain strict adherence to approved procedures, this QAPP, and relevant QAPjP requirements

- Assist hazardous waste coordinator

- Assist chemists

- Pass proficiency testing before analyzing samples.

\subsubsection{Other Organizations}

2.3.3.1 Quality Systems. The WMH Quality Systems organization has the following responsibilities:

- $\quad$ Provides external QA support services through the periodic auditing of SAS and other quality assurance and quality management consulting activities.

- As part of the Hanford Performance Evaluation Program, will lead the development and implementation of the Performance Evaluation Programs, as requested by SAS management. 
2.3.3.2 Waste Management Laboratory. SAS uses the services of the WML organization on a 1. work-order basis in the following areas.

2.3.3.2.1 Radiation Control. Radiation Control provides support personnel to SAS for establishing and monitoring personnel dose rates, conducting radiation and contamination surveys throughout the SAS facilities, establishing and verifying radiological postings, and writing and issuing Radiological Work Permits. Other duties include establishing and verifying radiological postings and providing radiological technical guidance to the SAS management.

2.3.3.2.2 WML Business Services. SAS uses Business Services to issue and track the Laboratory Analytical (LA) procedures, Laboratory Quality (LQ) procedures, and Laboratory Computer (LC) procedures. Electronic and hard-copy procedure distribution is completed by the Technical Support organization.

2.3.3.2.3 WML Standards Laboratory. The Standards Laboratory is responsible for the procurement and preparation of materials used for laboratory measurement system (LMS) calibration. When appropriate, these materials shall be traceable to a nationally or internationally recognized standard agency source (for example, National Institute of Standards and Technology [NIST]) or measurement system. Alternatively, the Standards Laboratory will procure materials of known quality and will document the materials as described in Section 2.3.3.2.4.

2.3.3.2.4 WML Maintenance Organization. The WML Maintenance Organization performs annual calibrations on SAS analytical balances and chart recorders. The Maintenance Organization also assists SAS in the identifierion ofidentifying non-data equipment.

2.3.3.3 WMH Genterator Services. The Generator Services group supports SAS on a taskorder basis providing services for ensuring that SAS complies with applicable local, state, and federal environmental regulations. The group prepares and/or reviews environmental permits for the facility and provides facility input to Sitewide environmental reports. The organization performs waste designation for laboratory analytical procedures and acts as a technical authority for waste management issues and procedures. The organization also provides environmental oversight and technical support. Applicable environmental regulations identified are Washington Administrative Code (WAC) 173-303; and WAC 246-247. The more specific Hanford Nuclear Facility Procedures are as follows:

- HNF-PRO-45I

- HINF-PRO-452

- HNE-PRO-453

- HNE-PRO-454

- HNF-PRO-455

- HNF-PRO-459

- HNF-PRO-462

- HNF-PRO-690
Regulated Substance Management NEPA, SEPA, Cultural and Natural Resources

Environmental Notification and Reporting

Inactive Waste Sites

Solid Waste Management

Environmental Training

Pollution Prevention

Applicable Environmental Statues, Regulations. 
2.3.3.4 Hanford Standards Laboratory. The Hanford Standards Laboratory (HSL) provides annual calibrations on various types of instrumentation used by SAS. Currently, SAS sends the equipment to the HSL to obtain calibration from an approved metrology organization. 


\section{NHC ORGANIZATION STRUCTURE}

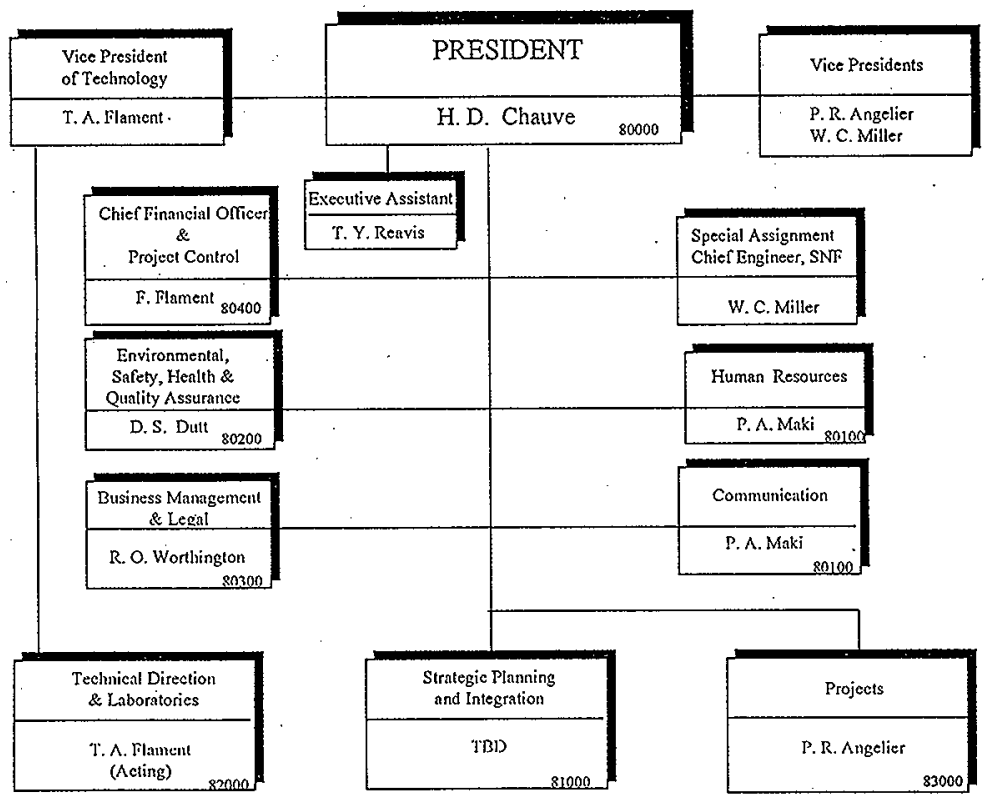

11. D. Clanıve 
Figure 2. Title. (ORG CHART)

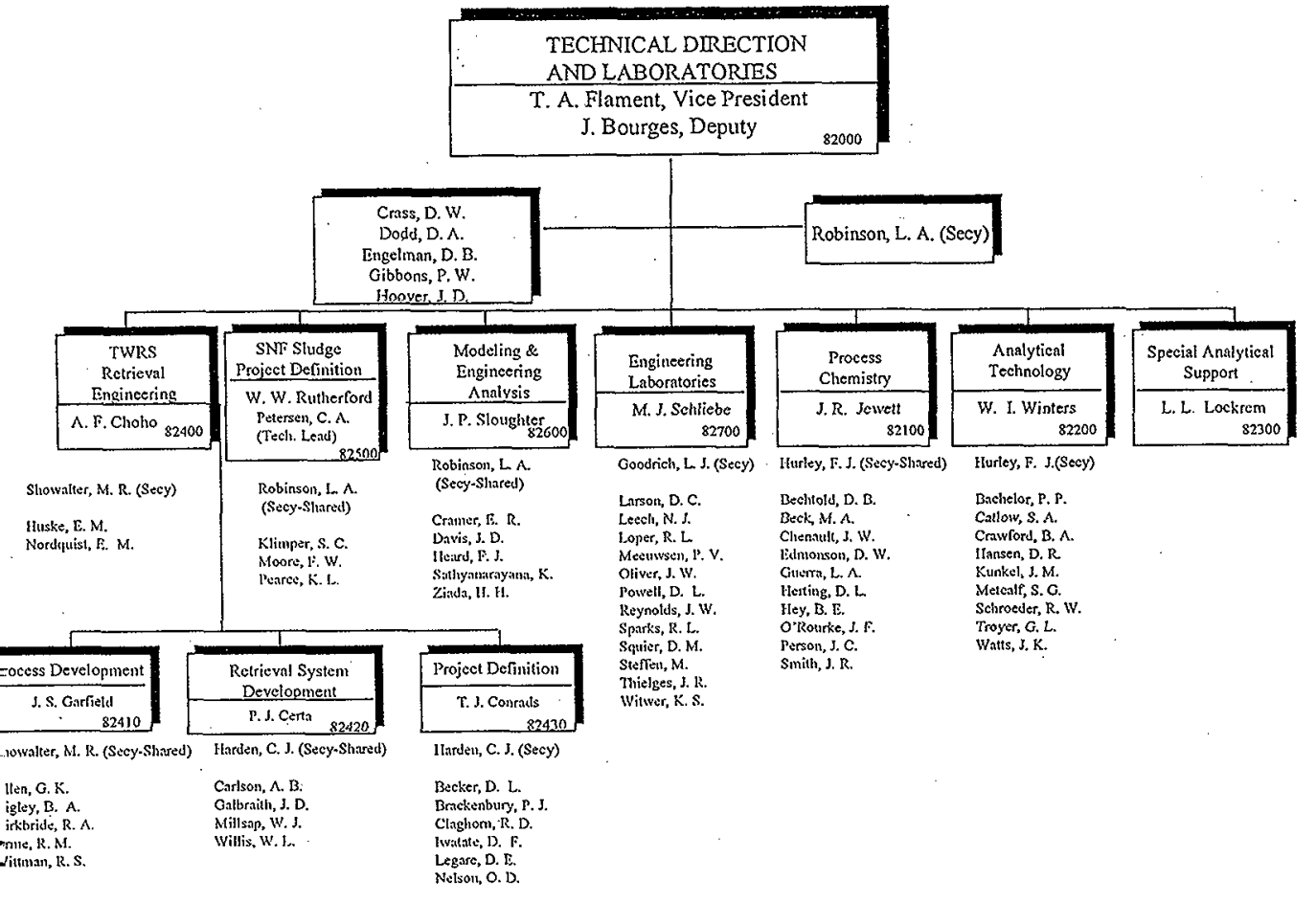


HNF-SD-WM-QAPP-034 Rev. 3

Figure 2. Title. (ORG CHART)
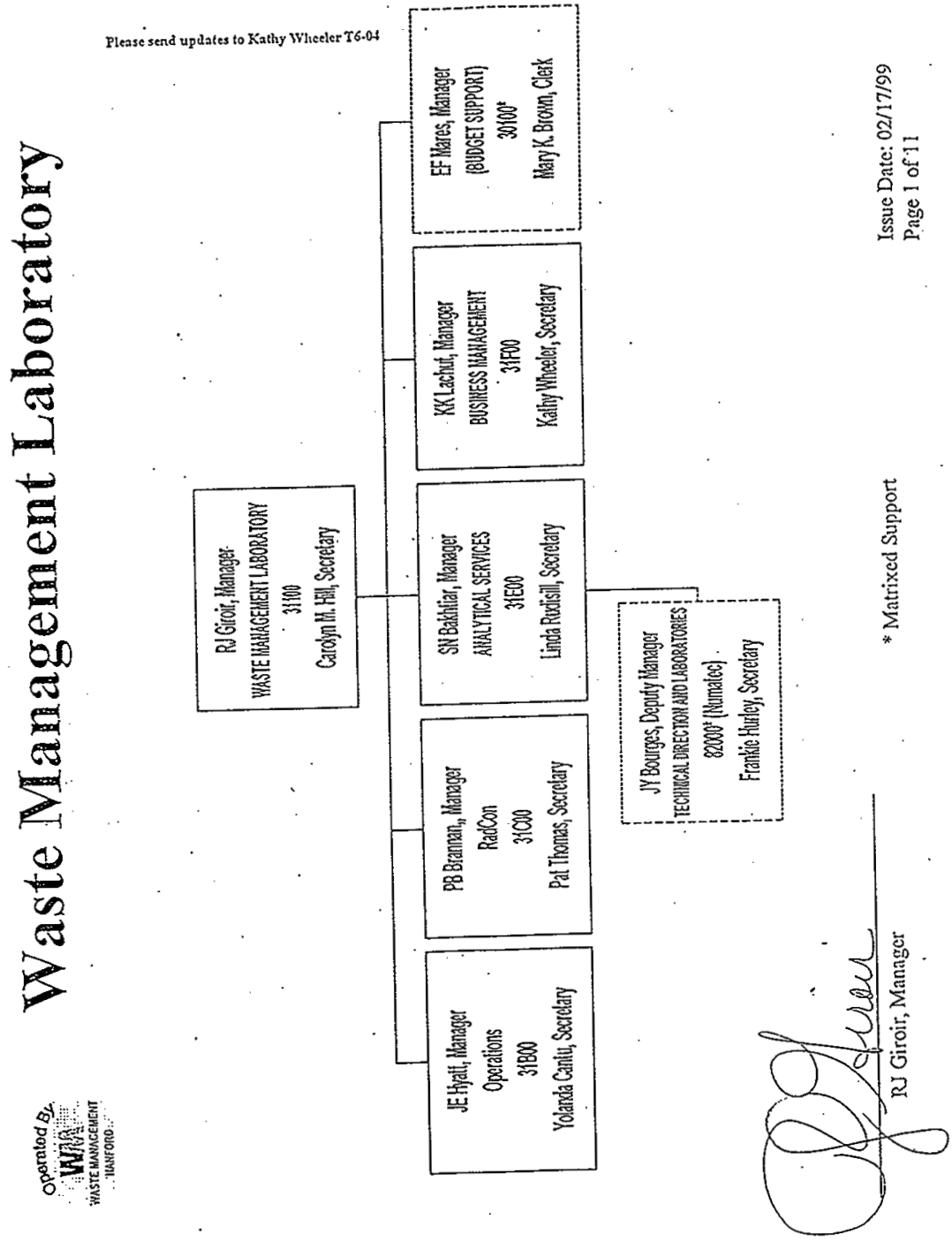

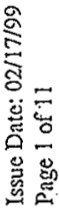


Figure 2. Title. (ORG CHART)

\section{oporated $\theta_{2}$ \\ Witis \\ WASTE MAMAGEHEIT HAMFORO \\ Waste Management Laboratory Technical Direction and Laboratories (Numatec)}
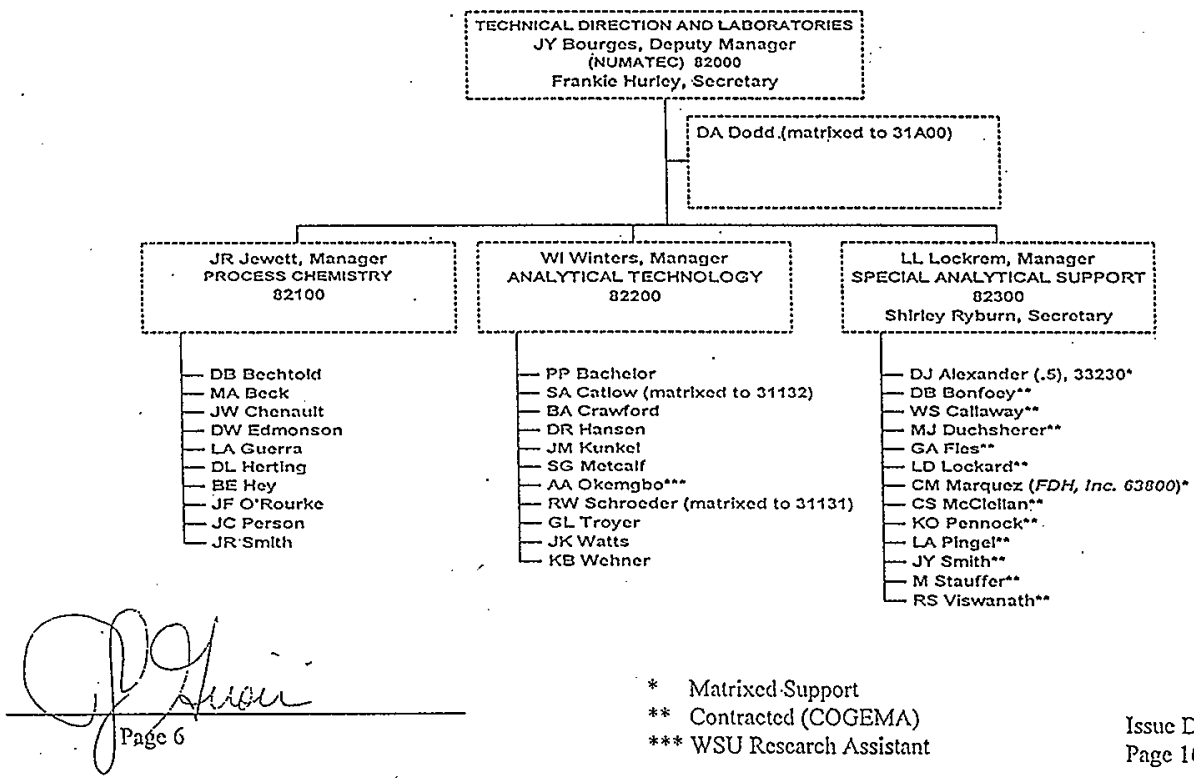

* Matrixed Support

** Contracted (COGEMA)

*** WSU Rescarch Assistant
Issue Datc: 02/17/99

Page 10 of 11 . 
HNF-SD-WM-QAPP-034 Rev. 3

Table 2-1. SAS Capabilities. (2 sheets)

\begin{tabular}{|c|c|}
\hline $\begin{array}{l}\text { WNTRUMENTS/ } \\
\text { METHODS }\end{array}$ & $\begin{array}{l}\text { ANALYTES/ } \\
\text { FUNCTIONS }\end{array}$ \\
\hline Airtoxics** & $\mathrm{VOA} * *$ \\
\hline Conductivity & Liquids and moist solids** \\
\hline Immunoassay & $\begin{array}{l}\text { PCB, pesticides, herbicides, total petroleum hydrocarbons, } \\
\text { poly aromatic hydrocarbons }\end{array}$ \\
\hline $\mathrm{GC} / \mathrm{FID}$ & VOA \& SV organic screens** \\
\hline GC/MS & VOA \& SV organic functional groups** \\
\hline $\mathrm{GC} / \mathrm{MS}$ & VOA by purge \& trap** \\
\hline GC/MS & $\mathrm{SVOA}^{* *}$ \\
\hline $\mathrm{IC}$ & Anions** Cations** \\
\hline $\mathrm{pH}$ & Liquids and some solids** \\
\hline $\begin{array}{l}\text { Scanning Electron } \\
\text { Microscope (SEM) }\end{array}$ & Elemental Analysis \& Physical Structure** \\
\hline TPH & Total Petroleum Hydrocarbons** \\
\hline XRF & Elemental Analysis** \\
\hline $\begin{array}{l}\text { Pensky-Martin \& } \\
\text { Setaflash }\end{array}$ & Flashpoint** \\
\hline Photovac & Total Organic Vapors \\
\hline FTIR & Fourier Transform Infrared-organics \\
\hline ICP & Metals \\
\hline Hazcat & RCRA Characteristics \\
\hline Bacteria & Coliform Membrane Filter \\
\hline BOD/DO Probe & Biochemical Oxygen Demand/Dissolved Oxygen \\
\hline Spectrophotometer & Inorganics \\
\hline DC ARC CD & Atomic Emissions-inorganics \\
\hline ISE & Ion Selective Electrodes, several inorganics \\
\hline TDS/TSS & Total Dissolved Solids Probe/Total Suspended/Gravimetric \\
\hline Corrosivity & Corrositex, Steel plate \\
\hline Moisture Balance & $\%$ Water \\
\hline
\end{tabular}


HNF-SD-WM-QAPP-034 Rev. 3

\begin{tabular}{|l|l|}
\hline $\begin{array}{c}\text { INSTRUMENTS/ } \\
\text { METHODS }\end{array}$ & \multicolumn{1}{|c|}{$\begin{array}{c}\text { Table 2-1. SAS Capabilities. (2 sheets) } \\
\text { ANALYTES/ } \\
\text { FUNCTIONS }\end{array}$} \\
\hline $\begin{array}{l}\text { Paint Filter Test } \\
\text { Cold Vapor Atomic } \\
\text { Absorption }\end{array}$ & Free Liquids \\
\hline PLM & Mercury \\
\hline TCLP/Toxicity & $\begin{array}{l}\text { Asbestos } \\
\text { Extraction }\end{array}$ \\
\hline Reactivity & \begin{tabular}{l} 
Cyanide/Sulfide \\
\hline
\end{tabular} \\
\hline
\end{tabular}




\subsection{PERSONNEL QUALTFICATION AND TRAINING}

SAS management shall determine the level of competence, experience, and training necessary to ensure satisfactory job performance, and document the minimum criteria required. Initial training requirements for each job category within the organization are given in the SAS Training Plan, HNF-3275 (Myers 1998), as well as in Section 3.3. Personnel shall possess qualifications that provide reasonable assurance that their decisions and actions will ensure that assigned responsibilities are conducted properly and safely. Years of experience often may equate to or outweigh academic achievement. Management is responsible for the documentation of personnel qualifications. Personnel shall be provided with continuing training to ensure that the required level of job performance is maintained.

Vendor and on-the-job training (OJT) be used to qualify personnel for method specifics. The analysis of laboratory standards within established control limits are used as the basis of proficiency testing and qualification.

To qualify on a particular method, an analyst shall demonstrate skill in performing the method by running a test sample and meeting previously established QC acceptance criteria.

\subsection{QUALIFICATION}

The management of SAS considers education, experience, and training as components of personnel qualifications. All personnel must meet or exceed minimum qualification requirements for their functional positions. Personnel qualification is based on program descriptions, which include job requirements and associated tasks and skills. Certain positions may also require OJT qualification in addition to formal classes. Course requirements for all personnel are electronically posted and tracked through the Training Matrix Program System (TMX) system. Refer to HNF. 3275, Special Analytical Support Training Plan, (Myers 1998) for job-specific qualification requirements.

\subsection{PERSONNEL SELECTION}

Employee selection and job assignment are based upon an evaluation of the requirements of the position and the candidate's education, training, and previous experience. Only those candidates meeting minimum requirements are selected.

\subsection{SAS GENERAL PERSONNEL TRAINING}

| The Special Analytical Support Training Plan, HNF-3275 (Myers 1998), identifies the course requirements for each position that are tracked through the TMX system. Individual training requirements are developed using program descriptions that identify job requirements and the associated tasks and skills for each position. 
In addition to formal classroom training and documented facility OJT, the SAS management encourages continuing improvement in the awareness and proficiency of all employees. Where

I practical, access to other PHMC and FDH, as well as offsite, training and professional development opportunities is encouraged.

SAS personnel are trained in basic facility orientation, general safety, building emergency procedures, and self-monitoring, if required. Training requirements described in this section are detailed in HNF-3275 and tracked on the TMX system. In addition to these mandatory training requirements, specific training related to each job category is described in the following sections. Additional training information is referenced in HNF-PRO-167 through HNF-PRO-179 and HNF-MP-599, Part 2, Section 2 (Beyers and Grier 1997).

Refer to WHM-200, Section 5.1, Table 1, "Minimum Position Requirements for Selected Positions." See Section 3.4.6 for specific instrument qualification requirements.

\subsubsection{Chemists/Scientists}

Chemist/scientist training includes operational specific procedures for working in the laboratory and procedure author training. Specific qualification records are used to document job positions. Technical staff qualification requires a scientific degree (baccalaureate degree), 2 years of job related experience, and 1 year of nuclear facility experience. Refer to WHM-200, Section 5.1, Table 1, "Minimum Position Requirements for Selected Positions." See Section 3.4.6 for specific instrument qualification requirements.

\subsubsection{Team Leads}

| Team Leads and Persons-in-Charge positions requires a high school diploma and 3 years of | nuclear facility experience. Refer to WMH-200, Section 5.1, Table 1, "Minimum Position Requirements for Selected Positions."

\subsubsection{Managers}

The role and responsibilities of a laboratory manager to lead, direct, and motivate people are emphasized. Managers are required to complete company and facility-specific training, as well as OJT. Facility training includes familiarization with the various safety, engineering, and work control systems in use at the SAS facility. Specific qualification records are used to document job positions. Managerial staff qualification requires a baccalaureate degree and 4 years of nuclear facility experience (education or experience that is job related may be substituted on a case-bycase basis). The degree may substitute for 3 of the 4 years of nuclear experience required on a one-for-one time basis). 


\subsubsection{Additional Job-Specific Training}

Additional job-specific course work may be required to develop and/or maintain proficiency in areas of hot cell operation, sample preparation, analytical instrumentation, maintenance, building operations, shift supervision, waste management, facility engineering, and OJT instruction.

The personnel qualification requirements are described in the following operational/instrument categories. These education/experience requirements meet or exceed the minimum transuranic (TRU) waste certification training and qualification requirements. Education levels may not be necessary if the immediate supervisor has a bachelor's degree in chemistry or a related field or if the analyst has the number of credit hours in chemistry courses required for a major in chemistry.

- Classical Laboratory Analyses

Education: Minimum high school diploma or equivalent (GED).

Experience: I year experience with classical chemistry laboratory procedures, preferred.

- Inorganic Sample Preparation

Education: Minimum high school diploma or equivalent (GED) or minimum of a college-level course in general chemistry

Experience: Minimum of 1 year experience in the preparation of environmental samples for the analysis of metals.

Minimum of 6 months experience in sample dissolution using microwave digestion techniques.

- Ion Chromatography (IC)

Education: Minimum of Bachelor's degree in chemistry or equivalent specialized training in IC.

Experience: Minimum of 1 year of applied experience in the operation of $\mathrm{IC}$ instrumentation.

- Atomic Absorption (AA) Spectroscopy

Education: Minimum of Bachelor's degree in chemistry or equivalent specialized training courses in flame AA or graphite furnace or cold vapor AA.

Experience: Minimum of 1 year of applied experience with AA instrumentation for flame, graphite furnace, and cold vapor AA. 
- Inductively Coupled Plasma (ICP) Spectroscopy

Education: Minimum of Bachelor's degree in chemistry or equivalent specialized training courses in ICP.

Experience: Minimum of 2 years of applied experience with ICP analysis of environmental samples.

- ICP/Mass Spectroscopy (ICP/MS)

Education: Minimum of Bachelor's degree in chemistry or equivalent specialized training courses in ICP/MS.

Experience: Minimum of 2 years of applied experience with ICP/MS analysis of environmental samples.

- Organic Sample Preparation

Education: Minimum of high school diploma or equivalent (GED).

Experience: Minimum of 1 year experience in extraction and concentration of environmental samples.

- Gas Chromatography (GC)

Education: Minimum of a Bachelor's degree in chemistry or specialized GC training courses. including Flame Ionization Detector/Electron Capture Detector training preferred.

Experience: Minimum of 1 year of applied experience with GC analysis of environmental samples.

- Gas Chromatography/Mass Spectroscopy (GC/MS)

Education: Minimum of Bachelor's degree in chemistry or equivalent training courses in GC/MS.

Experience: Minimum of 1 year of applied experience with GC/MS analysis of environmental samples.

- Headspace Gas Sampling/Environmental Sampling

Education: Minimum of Bachelor's degree in chemistry or equivalent as well as training courses in environemental sampling and EPA chain of custody and OJT in the field.

Experience: Minimum of 1 year of applied experience with sampling of headspace gas analysis and environmental samples. 
- Facility Quality Assurance Officer

Education: Minimum of associate of arts degree in chemistry or equivalent training courses as well as classes in environmental compliance, auditing and quality assurance-related coursework.

Experience: Minimum of 1 year of applied experience with laboratory quality assurance and quality improvement

The following components need to be considered for all training:

- Method

- Method chemistry

- QC built into the procedure

- Record keeping requirements

- Normal operation.

- Instrument

- Location and use of maintenance and performance logbooks

- Location of spare parts

- Mechanism for reporting/addressing physical or operational problems

- $\quad$ Facility utility tie-ins

- Waste management and safety/health hazards.

- Data

- Proper reporting of analytical results and data validation

- Proper shift turnover.

\subsection{CONTINUING TRAINING REQUIREMENTS}

Individual training requirements given in the Training Plan (Myers 1998) and the TMX system serve to identify and track initial and continuing training course requirements for employees.

Courses identified as part of the employee continuing training are typically required omanrannually or biennially. Employees are scheduled for courses identified on the TMX by the centralized WMH training organization. Continuing training programs are structured around specific position needs and are designed to enhance personnel proficiency and qualification.

If performance is consistently unsatisfactory for a particular aspect of work, the analyst shall be removed from that duty until initial qualification performance is again demonstrated. 


\section{| 3.5 ON-THE-JOB TRAINING}

In general, OJT training is designed to identify what a trainee should be thinking and what they should know when actually performing the procedure. On-the-job training makes use of competent, trained OJT instructors and evaluators. For the most part, SAS uses mentoring for OJT training. Mentoring is based on the trainee having a general basis of knowledge to be able to perform the duties, but needing specific training to complete the assignment. The majority of personnel within SAS are degreed in chemistry or a related field and are qualified based on subject matter expertise to perform OJT. OJT instructors/evaluators shall have related technical experience consistent with the material being presented. An OJT evaluator verifies a trainee's satisfactory completion of a performance or operational evaluation by signing the appropriate spaces on an OJT checklist. The OJT checklist is used to document the completion of training on laboratory analytical procedures.

\subsection{TRAINING RECORDS}

Individual employee training and required courses are recorded and tracked on the TMX system. Completion of external training outside the company may be recorded in the TMX system by submitting a "Training Completion Record Form C" (Form A-6000-821) to Training Records. Employees have resumest and competency records on file with their managers. These files may also contain completion certificates from special seminars, symposiums, or vendor training courses. In addition, specific job positions have qualification requirements through specific procedures, manuals, or other required reading needed for their jobs.

Training will be ongoing and documented by the use of training records. Training records shall 1 include but are not limited to the following:

- Personal resume

- TMX print out, to be updated quarterly

- Copies of certificates of training or continued education pertaining to the individual job category

- Letters of qualification

- Performance evaluations where applicable

- OJT training checklists

- Field training experience where applicable, i.e., sampling

- Copies of analyst's method detection limit determinations, performance evaluation sample results, where applicable 


\subsection{QUALITY ASSURANCE OBJECTIVES}

\subsection{DATA QUALITY OBJECTIVES}

Quality assurance objectives provide a set of recognized parameters to monitor performance of an analytical measurement system and to qualify analytical data. Establishment of data quality objectives criteria can be achieved based on regulatory requirements, (for example, RCRA, CERCLA, the Clean Air Act of 1955 (CAA) and the Clean Water Act of 1977 (CWA), HASQARD requirements, and data use (for example, regulatory, process control, screening, planning, and development).

Analytical parameters and properties that are selected for measurement under this QAPP will be identified in the individual site specific work plans, statement of work (SOW), sampling and analysis plans, treatability study work plans, project-specific work plans, remedial action plans, or the project-specific QAPjPs. These planning documents shall specify all analytical parameters. Cross referencing to analytical method requirements, maximum detection or quantitation limit values, and maximum acceptable ranges for precision and accuracy in all matrices of concern must be provided to enable data evaluation against reasonable limits or determination of acceptability. This information should be provided through a formal DQO process or a documented agreement between SAS and the client. The client-required precision, accuracy, and minimum reporting levels for work performed shall be provided before beginning analysis. In the event that a planning document is not issued, written correspondence between SAS and the client shall be placed into the project files. If none of thethis documentation exists, the analytical requirements of HASQARD and/or SW-846 (EPA 1992) shall be followed as a default.

The overall QA objective of this plan is to ensure that field sampling, chain of custody, laboratory analysis, and reporting will provide results that will meet the customer's requirements, meet the data quality objectives, and be defensible, as applicable. Specific procedures for sampling, chain of custody, laboratory instrument calibration, analytical method, reporting of data, internal quality control, audits, preventive maintenance of field equipment, and corrective actions are described in other sections of this QAPP and summarized in the QA Matrix Index, Table 1-2.

I This QAPP is designed to meet the following QA objectives. These requirements may vary based on individual customer needs.

- Provide effective control of data quality

- Ensure that quality-related documentation provides a valid and traceable record of control measures applied within SAS

- Ensure that sample documentation is complete

- Ensure that instrument calibration or performance check of a test method has been performed and is current. 
HNF-SD-WM-QAPP-034 Rev. 3

- Ensure that QC procedures prescribed in analytical methods have been followed.

- Ensure that analyses are represented with precision, accuracy, representativeness, comparability, and completeness as required by the customer requirements and the QAPjPs.

\subsubsection{System for Notification of Unique Data Quality Requirements}

A communication system is set up in the laboratory for notification of unique data quality requirements after the laboratory client agreement has been finalized. The communication system is set up in the following manner.

- Unique customer requirements will be identified on the chain of custody and relayed to the analysts and technical leads by the sample custodian who ensures the requirements are met.

- Alternatively, a special meeting with appropriate laboratory supervisor or managers, chemists, $\mathrm{QA} / \mathrm{QC}$ representativetst), and project coordinator(s) can be used.

\subsubsection{Client Complaints and Resolution}

Client complaints or issues will be documented by the program management and/or technical lead. The lead shall document the complaint or issue, the client contact, and the date the contact was made. The lead shall track the complaint or the issue until resolution. All subsequent information that is-used to resolve the complaint or issue will be maintained in a designated file in the QA file cabinet of the 622-F Facility, Room 3.

Issues involving the corrective action process, such as initiation of non-conformance reports (NCR) or Letters of Observation, will be documented and tracked through the deficiency tracking system (DTS). Examples of conditions where corrective action may be implemented are as follows:

- Failure to follow client analytical requests and/or tataquality objective (DQOs); this condition may be addressed as part of the case narrative in the data package.

- Documentation errors

- Discrepancies in chain of custody.

\subsection{CLIENT DATA QUALITY REQUIREMENTS}

Five parameters are often used by the client to define project data quality requirements. These are precision, accuracy, completeness, comparability, and representativeness. Of these, the-precision, 
accuracy, and representativeness have direct impacts on data quality (see Chapter 11 for limitations associated with precision and accuracy). The laboratory and client share responsibility for selecting appropriate sample preparation and analysis methods.

High radioactivity can limit the types of instrumentation that can be used and/or require alternative or modified instrumentation to reduce dose rate (time of exposure, distance to exposure) for the analysts at the bench. For example, ICP emission spectroscopy, IC, and chromatography instrumentation must be modified to allow the capture of all gas or waste discharges. This typically means physical modification to allow gas collection or capture in a hood.

The precision and accuracy requirements based on hte sample matrix shall be agreed upon by the laboratory and the client and should be based on the error tolerances (uncertainty) of the analytical methodology. Accuracy based on standards is controlled by the laboratory. The laboratory is responsible for providing precision and accuracy values obtained from the standards to the client. If the client has special requirements for precision and accuracy, the requirements must be identified in the agreement. If the client has no special requirement, SAS shall provide technical assistance in determining the requirements, which will meet or exceed the requirements of this document.

\subsubsection{Precision}

Precision is a measure of the degree that two or more measurements are in agreement. Field precision is assessed through the collection and measurement of field duplicates at a rate of one duplicate per 20 analytical samples, one per batch, or another interval based on customer requirements. Precision in the laboratory is assessed through the calculation of relative percent differences (RPD) and relative standard deviations (RSD) for two or more replicate samples.

The laboratory control sample also can be used to indicate precision. Spiked samples and duplicate spiked samples (called the matrix spike and matrix spike duplicate) are used to determine the precision of analytical measurements. Precision is obtained by calculating the RPD of the pair. When data isare outside the acceptance limits, the analyst will include a statement in the data report. The analyst will evaluate the data to determine if they requires additional analysis.

Acceptance criteria for precision shall be established for each project and agreed twon by the laboratory and the client. Acceptance criteria for each analyte or analyte method shall be listed in the project plan, characterization plan, SOW, or other work authorization document. SAS provides historical precision values to the client based on the measurements from the standards.

\subsubsection{Accuracy}

Accuracy is the degree of agreement between an observed value and an accepted reference value. Accuracy in the field is assessed through the use of field and trip blanks and through the adherence to all sample handling, storage, preservation, and holding times. Laboratory accuracy 
is assessed through the analysis of matrix spikes (MS) or standard reference materials (SRM) or some other material of known concentration. It is expressed as the percent recovery of the spiked sample. The difficult samples and a wide variety of sample matrices involved make the cost of determining accuracy based on sample matrices excessive; therefore this method will not be used..

Project-specific accuracy requirements based on standards shall be listed in the project plan, characterization plan, SOW, or other customer work authorization document. SAS can provide historical values to the client based on the measurements from the standards.

\subsubsection{Comparability}

Comparability is an expression of the confidence with which one data set can be compared with another. Comparability depends on the proper design of the sampling program and will be satisfied by ensuring that the sampling plan is followed and that proper sampling techniques are used. Planned analytical data will be comparable when similar sampling and analytical methods are used and documented. Split samples sent to separate laboratories for the same analysis will be used as comparability studies. All reported data will be calculated and delivered in units consistent with those DQOs set forth in the QAPP describing customer requirements. Guidelines for data reporting and quality assurance are the basis for SAS good laboratory practices.

\subsubsection{Completeness}

Completeness is a measure of the amount of valid data obtained from a measurement system compared to the amount that was expected to be obtained under normal conditions.

Field completeness is a measure of the number of valid measurements obtained from all the measurements taken in the project. Field completeness will be greater than 90 percent. Completeness is routinely calculated as a percentage factor based on the number of measurements judged valid versus the total number of measurements made. Quality Control limits will be based upon the mean precision and the mean accuracy values determined by SAS. Completeness should not be used as the sole measure of performance. Lack of completeness may be because of uncontrollable circumstances (that is, lack of sample, matrix interferences, and so forth).

\subsubsection{Representativeness}

Representativeness expresses the degree to which data accurately and precisely represents a characteristic of a population, parameter variations at a sampling point, a process condition, or an environmental condition. Representativeness depends on the proper design of the sampling program and will be satisfied by ensuring that the field sampling plan, letter of instruction or SOW is followed and that proper sampling techniques are used. The degree to which a sample accurately and precisely represents the condition under which it was taken, at the time and location of field sampling, is representativeness. Whenever possible, SAS will see that sample 
aliquots and distributions are evenly and uniformly divided so that reported results are representative. Representativeness will be ensured by using the proper analytical procedures, meeting sample holding times, and analyzing and assessing field duplicated samples.

Representativeness should be attempted by proper homogenization or appropriate subsampling (if different phases are apparently visible in the sample). Once subsampling occurs, identification of chemical and physical properties of subsamples, proper analytical protocol, and traceability of results to the original subsamples should be in place. Clients shall be consulted if unplanned subsampling appears necessary. 


\subsection{FIELD ANALYTICAL SUPPORT LEVELS}

The wide variety of quality control protocols associated with field analysis necessitate the use of multiple levels of data documentation QC. All levels of QC require that strict chain-of-custody protocol be maintained during the sampling, analysis, and disposal of material and/or waste. The QC levelslisted in Table 5-1; closely correspond to the documentation requirements of the EPA Contract Laboratory Program analytical support levels I through V. These levels are used as a tool to assist the customer in meeting DQOs with the appropriate levels of documentation and quality control.

QC Documentation Level A. Level A protocol requires only a chain of custody as the documented record.

QC Documentation Level B. Many field methods that use portable test kits employ level B QC analyses. As a minimum, the methods use a beginning standard and blank but other QC may be performed depending on the procedure. Data obtained using QC level B is reported in summary format and all associated raw data is maintained by SAS in accordance with HNF-PRO-210, Records Management Program.

QC Documentation Level C. This level of QC is comparable to "equivalent" SW-846 (EPA 1992) and Standard Methods procedures. Included in the protocols are chain of custody, initial and continuing calibration verification standards (ICV/CCV), blanks, (MS), and laboratory control standards (LCS). Data obtained using QC level $\mathrm{C}$ are reported in summary format. All associated raw data is maintained by SAS in accordance with HNF-PRO-210, Records Management Program.

QC Documentation Level D. Level D protocols include the same QC criteria and frequency as Level C. In addition, copies of raw data are included in the final report submitted to the customer.

QC Level E. In addition to chain of custody, calibration checks, and blanks, level E includes customer specified QC as well as custom reporting formats. The required level of QC and reporting will be determined during the development of DQOs for the project and should be specified in the sample analysis plans (SAP) and QAPjPs.

I NOTE: Because of the nature of the samples, matrix spikes will not be performed on vapor samples. 
HNF-SD-WM-QAPP-034 Rev. 3

Table 5-1. Quality Control Level Requirements.

\begin{tabular}{||l|c|c|c|c|c||}
\hline & Level A & Level B & Level C & Level D & Level E \\
\hline Chain of custody & $\mathrm{X}$ & $\mathrm{X}$ & $\mathrm{X}$ & $\mathrm{X}$ & $\mathrm{X}$ \\
\hline Blanks & & $\mathrm{X}$ & $\mathrm{X}$ & $\mathrm{X}$ & $\mathrm{X}$ \\
\hline ICV/CCV & & $\mathrm{X}$ & $\mathrm{X}$ & $\mathrm{X}$ & DQO/QAPjP \\
\hline Duplicates & & & $\mathrm{X}$ & $\mathrm{X}$ & DQO/QAPjP \\
\hline Matrix Spike & & & $\mathrm{X}$ & $\mathrm{X}$ & DQO/QAPjP \\
\hline LCS & & & $\mathrm{X}$ & $\mathrm{X}$ & $\mathrm{X}$ \\
\hline Summary Report & & $\mathrm{X}$ & $\mathrm{X}$ & $\mathrm{X}$ & $\mathrm{X}$ \\
\hline Full Deliverables Report & & & & $\mathrm{X}$ & $\mathrm{DQO}$ \\
\hline \hline
\end{tabular}




\subsection{SYSTEMS QUALITY ASSURANCE}

\subsection{SOFTWARE SYSTEMS QUALITY ASSURANCE}

Laboratory software systems can be separated by application into two categories: administrative and technical. Administrative software systems manage the work flow or monitor performance against administrative requirements. Examples of administrative software systems are those that control sample tracking, procedure control, training, and reporting. Technical software systems control laboratory systems, and accumulate and reduce data. Examples of technical software systems are those that provide instrument interface, calculations, calibration control, and control charts.

\subsubsection{Control Requirements}

Software control requirements applicable to both commercial and laboratory-developed software shall be developed, documented, and implemented. Software systems shall be protected from unauthorized or inadvertent changes.

Software systems shall be documented under configuration control. For laboratory-developed software systems, a copy of the original program code shall be maintained, and all changes shall include a description of the change, authorization for the change, and test data that validates the change. Configuration control and acceptance test data shall be maintained for commercial software packages.

HNF-PRO-309, Control of Computer Software, governs laboratory software system configuration control.

\subsubsection{Acceptance Testing}

Software systems (excluding technical software as instrument specific and vendor supplied) shall be tested for acceptance when installed, after changes, and periodically during use. The frequency of the test shall be based on the potential for adverse impact on the laboratory and the ease with which changes can be made to the computer code. Testing may consist of manually performing calculations, checking against another software system that has been tested, the analysis of standards, and/or the use of hand calculations, calculations using comparable proven programs; or empirical data and hand application used in calculations shall verify the spreadsheet application before initial use and after modifications.

Documentation of the testing shall include the test cases, printouts of the data or results from data generated by the software for comparison, the name of the person performing the test, and the date the test was performed. The version of the software and the manufacturer also must be documented. Software control is the responsibility of each individual as well as management. All 
paperwork generated during software verification such as computer printouts and supporting calculations shall be maintained as quality records.

The team lead must verify laboratory-developed software, including spreadsheets used for data reduction, must be verified initially and after all changes. Verification testing may consist of manually performing calculations or checks against another software product that has been tested or by the analysis of standards.

Commercially-available software is acceptable as supplied by the vendor.

\subsubsection{Backups}

Both software and data shall be backed up. The frequency of backup shall be based on the amount of data and the impact of the loss of data or software on the organization. The backups are considered a quality record.

\subsubsection{User's Manuals}

Laboratory computer (LC) procedures are issued when appropriate for an application related to computers or computer software. If programs or applications for projects are small or straightforward, a controlled manual or special training is not required (see HNF -PRO-433, Small Job Development). SAS maintains copies of the software user manuals in the SAS library.

\subsubsection{Error Reporting}

Data management software errors found during use are immediately reported to the system administrator of software, technical manager, production manager (to determine affected data previously reported), QA and the vendor, if applicable. Errors and corrective actions shall be documented. Information related to discovery and notification of technical instrument software errors shall be documented in the appropriate laboratory notebook. Corrective actions shall be documented using NCR's or Letters of Observation, and tracked with DTS.

\subsection{TECHNICAL/ADMINISTRATIVE SYSTEMS}

Technical systems ensure that the techniques used are applicable and properly applied. These systems shall include sample exchanges, standards programs, control of standards and reagents, data reduction and reporting, data verification, and technical systems assessments.

Fluor Daniel Hanford, Inc., maintains an administrative control system based on a hierarchy of CMs. Control Manual's are being phased out and reissued as Hanford Nuclear Facility Procedures. These procedures provide documented interpretation of DOE orders and procedures for implementation. Contractors such as WMH implement FDH directives through WMH-100 
and WMH-200 level documents. These policies provide direction for implementation of key policy and administrative actions, based on specific DOE orders (for example, safety, quality assurance, and purchasing). In addition to the administration directions listed in HNF and WMH documents, laboratory prócedures are used for specific activities. HNF-CM-5-4, Laboratories Administration, provides the documentation describing and directing laboratory activities not sufficiently covered in WMH or HNF documents. Eventually, HNF-CM-5-4 procedures will be captured in the WMH-310 manual and the HNF-CM-5-4 version will be deleted.

\subsection{PHYSICAL FACILTTES SYSTEMS}

SAS facilities consist of several fixed and mobile laboratories. Table 6-1 identifies each laboratory and its function. Rooms including conference, storage, computer, lunch, locker/change rooms, office areas, and analytical laboratories are located in the $622 \mathrm{R}$ facility. Mobile laboratory trailers are stored adjacent to the $622 \mathrm{R}, 622 \mathrm{~F}, \mathrm{MO}-254$, and $\mathrm{MO}-255$ facilities. The MO-254 and MO-255 trailer includes a lunchroom and cubicle offices. The $622 \mathrm{R}$ facility is designed to handle non radioactive samples and the mobile laboratory trailers are designed and designated to handle radioactive samples. The physical layout of the SAS facility allows for deployment of mobile laboratories, installation of monitoring equipment, and access by personnel to deploy hand-held monitoring equipment.

The manager and/or appointee will inspect laboratory facility conditions quarterly at a minimum, and document deficiencies for corrective actions on safety/housekeeping inspection forms. WMH-200, Section 1.13, "Health and Safety Self-Inspection Program," is used for performing health and safety inspections of the SAS facilities.

\subsubsection{Power}

Power is a major concern for in situ and field analysis. All analytical equipment requires dependable power. Mobile laboratories require considerable power to operate analytical equipment. When line power is not available, generators are used. Power from generators requires conditioning before it is used to power analytical equipment. SAS generally runs generator power through an inverter uninterruptible power supply to produce clean line voltages and frequencies.

\subsubsection{Safety}

All SAS facilities, including mobile analytical facilities, are maintained in a safe manner. Appropriate safety equipment is maintained in the mobile laboratories to include; a minimum of one eye wash, appropriate spill kits, safety glasses, a fire extinguisher, and first aid kits. When in situ analyses is performed, portable eye wash stations and appropriate spill kits are placed near the point of analyses. Entry into the mobile facilities is limited to approved personnel. 
Table 6-1. Facility Identification.

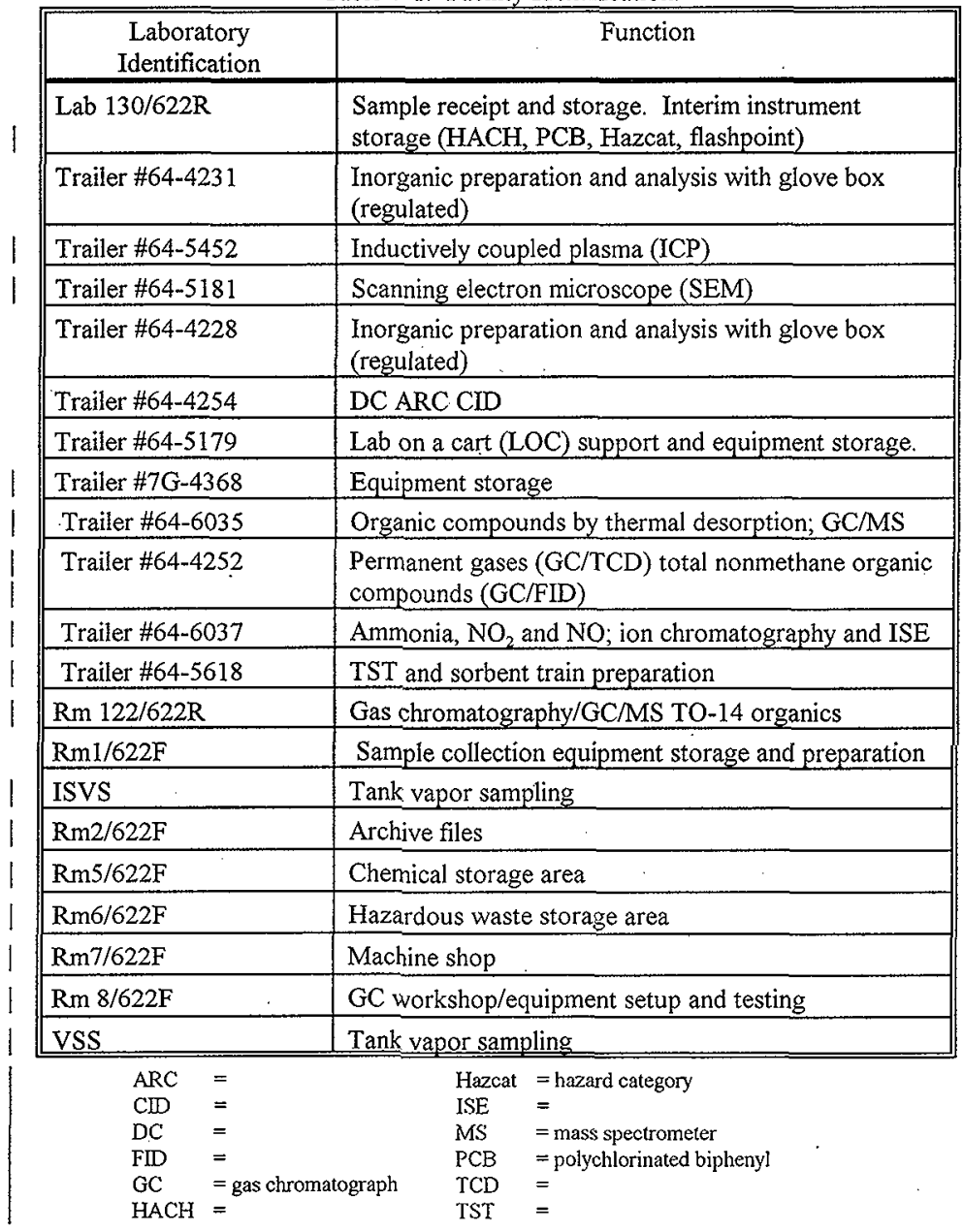




\subsection{SAMPLING METHODS}

\subsection{SAMPLING PROCEDURES}

Documentation of sampling procedures is critical to the technical defensibility and the legal defensibility/admissibility fo the resulting data. Whenever possible, industry recognized sampling methods from agency published source documents, such as DOE, EPA, and American Society for Testing Materials (ASTM) shall be employed. Sample collection and processing procedures may also use methods published by the U.S. Geological Survey, U.S. Department of Agriculture, and professional groups such as the American.Water Works Associate. Current DOE, EPA, and ASTM sampling methods are detailed in the following sources

- DOE, Methods for Evaluating Environmental and Waste Management Samples, DOE/EM-0089T, October 1992

- DOE, EML Procedures Mamual, 27th Edition (Volchok 1992)

- DOE, Environmental Survey Manual, Appendix E, "Field Sampling Protocols and Guidance, Office of Environmental Audit," 1987

- EPA, Representative Sampling Guidance, Vol. 1, Soil, 1991

- EPA, Soil Sampling Quality Assurance User's Guide, 1989

- EPA, Sampling of Water and Wastewater (Shelley 1977)

- $\quad$ EPA, Compendium of Superfund Field Operations Manual, 1985

- ASTM, Sampling Surface Soils for Radionuclides, ASTM C-998-83, 1983

- ASTM, Standard Practices for Sampling Water, Method D 3370-76, 1977

- WAC-173-303-110(2), "Dangerous Waste Regulations."

\subsection{SAMPLE CUSTODY AND HANDLING}

\subsubsection{Sample Security and Chain of Custody}

The 622R facility, 622F Sample Storage Area, and all mobile laboratories are secured facilities, restricted to authorized personnel only. During the day, exit doors are either monitored by authorized personnel or locked. All entrance/exit doors are locked after normal working hours. All facility doors are secured with either OMNI or key locks. Information can be downloaded from the OMNI locks and a print out of all personnel who have entered the facility can be 
obtained. Visitors and guests are required to $\log$ in at the front desk and are escorted when in the laboratory facility. SAS follows WMH-310, Section 1.4, "Laboratory and Sample Security."

A chain-of-custody will be initiated upon sample collection or receipt of a sample. In some cases, this could include preparing sample bottles and adding preservatives before sampling. Chain of custody will be maintained from the time of sampling or receipt until the sample is destroyed by analysis, disposed of, or returned to the customer. Internal chain of custody is documented in the Custody Logbooks located at each sample storage area.

Custody in the laboratory is defined as secured to prevent tampering and may be accomplished by ensuring that the sample beis in one of the following situations:

- In actual physical possession

- In view of the sample custodian after being in physical possession.

- In a locked area

- In a designated secured area accessible only to authorized personnel.

\subsubsection{Sample Custodian}

The duties and responsibilities of the sample custodian shall include but are not limited to the following:

- Inspecting sample shipping containers

- Verifying the information recorded on the chain-of-custody form

- $\quad$ Receiving samples

- $\quad$ Signing appropriate documents shipped with the samples

- Marking and/or labeling samples with appropriate laboratory number

- Placing samples into the appropriate storage/security area

- Initiating sample analysis by notifying the appropriate personnel and relaying unique customer requirements

- Recording samples in the internal chain-of-custody logbook

- Always maintaining the liquid level in all thermometer flasks in the refrigerator at more than half full.

Chain of custody is maintained as required between the sample collection, transportation, and receipt. Internal custody refers to maintaining custody as the sample is dispersed to various 
personnel within the laboratory for analysis. Internal sample custody is maintained until disposal of the sample from the laboratory. The following procedures cover sample custody and handling:

- WMH-310, Section 2.13, "Preventing Sample Contamination"

- LO-090-450, Sample Receiving, Custodianship and Return

- LO-100-400, SAS Waste Management.

- $\quad$ HNF-PRO-455, Solid Waste Management

\subsection{HOLDING TIMES}

The majority of work supporting RCRA and CERCLA requires adherence to holding time requirements. These holding times begin when the sample is collected. Holding time is understood to be the time between sample collection and preparation and/or final analysis. Regulatory holding time criteria range from 24 to 48 hours for some inorganic tests, to 1 to 2 weeks for most organic analyses, and up to 6 months for most metals and radionuclides. The shorter holding times required for some tests frequently will not be met because of the increased time required to handle high-radiation samples with remote handlers in hot cells. The increased logistics required to survey, transport, and screen the samples before analysis wilt also will increase the time between sample collection and analysis.

Tables 7-1 and 7-2 lists regulatory required holding times for the EPA or Contract Laboratory Program (CLP) for projects that must comply with either RCRA or CERCLA regulations. Project requirements will supersede the requirements listed in Tables 7-1 and 7-2.

All holding time agreements should be based on SW-846 (EPA 1992) or CLP specifications and shall be established between the laboratory and client before sample analysis. If the laboratory is unable to meet prescribed holding times, (for example, because of sample radioactivity), the client must agree, in writing, as applicable, to this fact before work begins. The client is responsible for ensuring the timely delivery of samples to the laboratory to enable laboratory personnel meet holding time requirements. Holding time issues are addressed in the data package case narrative (if applicable).

The team lead is responsible for identifying holding times with the clients and notifying the responsible manager, chemist, and sample custodian before sample arrival. The sample custodian is responsible for notifying the laboratory personnel (responsible manager and chemists) upon receiving samples. After sample receipt, the responsible manager and chemist are responsible to meet the holding times requirements. 


\subsection{SAMPLE RECEIVING}

When sample receipt is completed, samples are then accepted for analysis. On acceptance of samples, the sample custodian shall sign the chain-of-custody and shall initiate internal chain of custody for analytical activities.

If prompt client response (within two business days) is not received to a notification of nonconformance, the laboratory can proceed at its discretion to analyze the samples. The analysts must document all actions and decisions in the project file, and include a summary of the nonconformances and their actions in the case narrative accompanying the final report.

\subsection{SAMPLE LOG-IN AND TRACKING}

Internal chain of custody is initiated by sample log-in and remains unbroken until sample disposal is completed. The sample custodian(s) is responsible for maintaining custody of the samples during the initial log-in processes. The sample custodian also is responsible for ensuring that all records documenting that possession are properly completed and placed in the laboratory record system. Additional information such as radiation level should be provided by the field sampler and documented on the chain-of-custody form. The team lead is responsible for checking the information related to storage, preservation, holding time, and requested analysis to make sure it matches the work-authorizing document (for example, Tank Sample Analysis Plan).

\section{Sample Preservation}

Most approved methods, including SW-846 (EPA 1992), require samples to be cooled to $4{ }^{\circ} \mathrm{C}$ while awaiting analysis. Samples of liquids require acidification to a $\mathrm{pH}$ of 2 for preservation before most metal analyses. The high radiation levels for many of the samples on the Hanford Site will require that different procedures be used. Appropriate changes in the data validation protocols are implemented to address these issues.

\subsection{LABORATORY INTERNAL CHAIN OF CUSTODY}

Procedure LO-090-450 Sample Recieving, Custodianship and Return, (Smith 1998) addresses sample receiving and custodianship for SAS. The internal chain-of-custody remains unbroken using a sample log-in and log-out system. The location of all samples and the samples custodian is traceable from the time samples are received at the laboratory until the analysis is completed and the sample is disposed of or returned to the cilent. SW-846 or CLP samples in custody of the analyst, shall not be left unattended. 


\subsection{SAMPLE DISPOSAL}

Sample disposal includes disposing of or returning the original samples to the client. The sample custodian is responsible for preparing a list of a client's samples that have been analyzed and can be disposed of after the clients agrees. The Generator Services, Environmental Compliance organization, support personnel and/or sample custodians are responsible for disposing of samples that have been relinquished from the laboratory.

\subsection{WASTE DISPOSAL}

Hazardous waste generated by analytical processes must be managed in a controlled, safe, efficient manner. The WAC) 173-303 mandates the management of hazardoustor dangerous waste generated by SAS. All personnel are responsible for ensuring that waste generated within their assigned area is managed in accordance with waste handling procedures. All laboratory employees are trained to the "SAS Waste Management." The drivers of these safety and environmental requirements are WAC 173-303, "Dangerous Waste Regulations;" OSHA 1910.120 (Haz Waste Ops), Chemical Hygiene 1910.1450, DOE Order 5820.2A, Radioactive Waste Management; the Title 49 CFR series of regulations for the Department of Transportation (Packaging of Waste); and HNF-PRO-451, Regulated Substance Management, HNF-PRO-453, Environmental Notification and Reporting, HNF-PRO-455, Solid Waste Management, HNFPRO-459, Environmental Training, HNF-PRO-462, Pollution Prevention, HNF-PRO-690, Applicable Environmental Statues, Regulations.

Waste generated during many operations can be predesignated to allow routine disposal from satellite accumulation. If the waste is not predesignated, the waste must be designated before being disposed of and an inventory is required during collection of the waste. WMH-370, Section 2.15, "Waste Predetermination." is used for predesignating waste at SAS.

Requirements for managing satellite accumulation areas, 90-day accumulation areas, and the treatment, storage, disposal (TSD) units (storage boxes) at the SAS facility, and other designated areas are documented in procedure LO-100-166, Manage Satellite Accumulation Area, 90-Day Accumulation Area, and the Treatment, Storage, Disposal (TSD) Units. 
Table 7-1. Holding Times for Aqueous Matrices:

\begin{tabular}{|c|c|}
\hline Analytes & Holding times \\
\hline Chloride & 28 days \\
\hline Cyanide (total and amenable) & 14 days \\
\hline $\mathrm{pH}$ & $24 \mathrm{hr}$ \\
\hline Chromium VI & $24 \mathrm{hr}$ \\
\hline Mercury & 28 days \\
\hline Metals (except chair VI \& Hg) & 6 months \\
\hline Nitrate & $48 \mathrm{hrs}$ \\
\hline Sulfate & 28 days \\
\hline Sulfide & 7 days, add zinc acetate \\
\hline Organic carbon, total (TOC) & 28 days \\
\hline Radiological test (alpha, beta, and radium) & 6 months \\
\hline
\end{tabular}


HNF-SD-WM-QAPP-034 Rev. 3

Table 7-2. Holding Times for Organic Analyses: Based on Regulatory Requirements.

\begin{tabular}{|c|c|c|}
\hline $\begin{array}{l}\text { Analytes or } \\
\text { Method of } \\
\text { Extraction }\end{array}$ & $\begin{array}{c}\text { CLP (OLM03.1) } \\
\text { Contracted Holding Times } \\
\text { Number of days for extraction/number of } \\
\text { days for analysis }\end{array}$ & \begin{tabular}{|c|} 
SW-846 \\
Holding times \\
Number of days for \\
extraction/number. of days for \\
analysis
\end{tabular} \\
\hline Volatile organics & $\begin{array}{l}10 \text { days for extraction and analysis after } \\
\text { validated time of sample receipt (VTSR). } \\
\text { Vapor- }\end{array}$ & $\begin{array}{l}14 \text { days for extraction and } \\
\text { analysis. }\end{array}$ \\
\hline $\begin{array}{l}\text { Semi-volatile } \\
\text { organics }\end{array}$ & $\begin{array}{l}\text { Water samples: } 5 \text { days after VTSR/within } \\
40 \text { days after extraction. } \\
\text { Soil samples: } 10 \text { days after VTSR/ within } \\
40 \text { days after extraction. } \\
\text { Vapor- }\end{array}$ & $\begin{array}{l}\text { Water samples: } 7 \text { days/ } \\
\text { within } 40 \text { days after } \\
\text { extraction. } \\
\text { Concentrated waste samples, } \\
\text { soil/ sediments, and sludges: } \\
14 \text { days/ within } 40 \text { days after } \\
\text { extraction. }\end{array}$ \\
\hline $\begin{array}{l}\text { TCLP extraction } \\
\text { (hazardous waste } \\
\text { toxicity) }\end{array}$ & N/A & 7 days \\
\hline $\begin{array}{l}\text { Pesticides, } \\
\text { chlorinated or } \\
\text { organophosphorus } \\
\text { (SW-846 method), } \\
\text { PCBs } \\
\text { (SW-846 method) }\end{array}$ & N/A & $\begin{array}{l}\text { Water samples: } 7 \text { days/ } \\
\text { analyze within } 40 \text { days after } \\
\text { extraction. } \\
\text { Concentrated waste samples, } \\
\text { soil/sediments, and sludges: } \\
14 \text { days/analyze within } \\
40 \text { days after extraction. }\end{array}$ \\
\hline $\begin{array}{l}\text { Pesticides } \\
\text { including PCBs } \\
\text { (CLP method) }\end{array}$ & $\begin{array}{l}\text { Water samples: } 5 \text { days after VTSR/within } \\
40 \text { days after extraction. } \\
\text { Soil Samples: } 10 \text { days after VTSR/within } \\
40 \text { days after extraction. }\end{array}$ & N/A \\
\hline
\end{tabular}

NOTE: Holding times for headspace gas analysis shall be specified in the project specific SAPS, QAPjPs or other governing documents.

CLP = Contract Laboratory Program

$\mathrm{PCB}=$ polychlorinated biphenal .

QAPjP $\quad$ = quality assurance project plan

SAP $\quad=$ sample analysis plan

TCLP = toxicity characteristics leaching procedure 
HNF-SD-WM-QAPP-034 Rev. 3

This page intentionally left blank. 


\subsection{CALIBRATION}

This section describes the calibration practices used by SAS. These practices include the following:

- Record keeping for calibration data

- Calibration of balances, thermometers, and pipettes

- Traceability and documentation of standards used in calibration

- Calibration of laboratory measurement systems.

The initial and continuing verification of laboratory measurement system $Q C$ are described in Chapter 12.

\subsection{CALIBRATION RECORDS}

SAS maintains calibratión records for all methods requiring laboratory measurement system (LMS) calibration. These records include raw data (for example, instrument response values necessary to reconstruct the calibration, such as peak areas, counts, absorbance values, or emission intensity), the standard used, corresponding concentration or activity data, calculated calibration factors (for example, regression results), criteria used to accept or reject the calibration (for example, correlation coefficient), effective date of the calibration, and analyst's name or initials.

Calibration records are maintained in logbooks, notebooks, or electronic files, as appropriate. When completed, these records are maintained in accordance with the process described in Chapter 10.

Results for sample analyses shall include the analysis date. This date and the recorded effective calibration date permit traceability of the analysis to the most recent preceding calibration. If ambiguity is possible because a calibration was performed after a sample analyses on the same date, either time-of-day information must also be recorded (for both calibration and analyses), or the date of the applicable calibration must accompany analysis results.

\subsection{BALANCES, THERMOMETERS, AND PIPETTES}

\subsubsection{Balances}

Balance calibration checks are performed annually, at a minimum, by an Instrument Specialist in the WML maintenance organization. Balances not passing these checks are removed from service. Balances that are moved must be recalibrated. They shall be tagged with a green "service requested" tag to remove them from service until they are recalibrated by the instrument specialist. 
The calibration of balances is verified, at a minimum, before use or-daily. The results of the I before-use checks are logged in a notebook maintained at the scale location. This notebook also contains acceptance criteria for each logged balance. If the check fails, the balance is tagged and is-taken out of service until it is recalibrated.

\subsubsection{Thermometers}

Thermometers and thermocouples used for sample storage refrigerators are checked at least annually against a nationally recognized standard (for example, NIST certified thermometers). HNF-IP-1 128, 2.14, "Thermometer Calibration," is used by SAS for the yearly calibration.

\subsubsection{Pipettes, Burets, and Dispensers}

- The calibration of pipettes and dispensers used for quality-affecting determinations is checked by weight daily or before use. The results of these checks are documented chronologically per pipette or documented based on which pipettes were used. Devices found to be out of calibration are removed from service.

- Pipette and dispenser performance is also is monitored by batch through their use for preparing LCS or blank spikes. Performance of the LCS is monitored by control charts. Acceptable performance of LCS ensures that the pipettes and/or dispensers used for the test are performing adequately. A failure of LCS results in an investigation of mechanical pipette and dispenser accuracy, validity of standards, instrument calibration, analyst practices, and other potential causes. Laboratory control sample failures related to pipetted dispenser performance will be adequately documented.

Pipette calibration information is entered into logbooks. Pipettes do not receive a calibration sticker. Each pipette has a unique control or serial number.

\subsection{GENERAL REQUIREMENTS FOR STANDARDS}

The WML Standards Laboratory is responsible for the procurement and preparation of materials used for LMS calibration. In some instances, SAS will procure standards from a vendor. When appropriate, these materials shall be traceable to a nationally or internationally recognized standard agency source (for example, National Institute of Standards and Technology [NIST]) or measurement system. Alternatively, the Standards Laboratory and SAS will procure materials of known quality and will maintain the certificate of analysis as a quality record. When these records are maintained, the final standard shall be considered traceable to the original standard reference material. Some standard materials that have a long shelf life may be recertified using LO-150-061, Criteria for the Recertification of Standards. 
Calibration standards that have exceeded their expiration date or shelf life shall not be used for LMS calibration and shall be placed in an appropriate waste management area or clearly marked $\mid$ as unusable for calibration purposes unless recertified, whichever is appropriate.

Standards that are prepared and used for the first time need to be verified against existing working standards or against an independent source to ensure accuracy of the standards. When primary

I standards analyzed for the first time cannot meet QC parameters, the problem must be investigated to identify the source of the problem, and must be documented. The supplier must be notified if the source or error originates from the supplier.

\subsection{CALmRATION OF LABORATORY MEASUREMENT SYSTEMS (LMS)}

The requirements for calibration of each LMS are included in the laboratory procedures that govern its operation. Calibration checks are performed to verify that the instrument is operating within required limits. If not, the instrument must be recalibrated. Instruments are not allowed to be used for sample analysis until they meet acceptance criteria. Calibration frequencies are based on the following parameters:

- Requirement of the published method

- Elapsed time since previous calibration

- Instrument conditions during usage

- Frequency of usage

- Inherent stability of the instrument

- Periodic maintenance

- Troubleshooting

- Actions to be taken if calibration fails acceptance criteria.

Any variances from the requirements included in the laboratory procedures must be based on an agreement between the laboratory and the client (for example, via letter of instruction, tank characterization plan, statement of work).

Specific requirements or considerations for calibration and calibration standards used for radiochemistry and inorganic, organic, and physical testing laboratory measurement systems are defined in Volume 4, Section 4, of the HASQARD. Appendix B lists recommended QA/QC calibration requirements.

Instruments that fail acceptance criteria shall be investigated and recalibrated. The responsible chemist or manager is required to take corrective action when measurement systems fail calibration QC criteria as demonstrated by the procedures.

\subsubsection{Inorganic Analysis}

Instrument calibration should be performed based on manufacturer's recommendations and should establish a working response range. Accuracy of the calibration shall be confirmed by performing an initial calibration verification immediately after calibration. The performance of an instrument 
measurement system during an analytical run shall be verified by a continuing calibration verification.

The responsible chemist is required to notify the facility quality assurance officer and to take corrective action when measurement systems fail calibration QC criteria as demonstrated by the procedures.

\subsubsection{Organic Analysis}

Instrument calibration shall be performed to establish a working response range. Calibration requirements for frequency, criteria, and corrective action for organic analysis are provided in HASQARD, Volume 4, Section 4. The calibration accuracy shall be confirmed by performing an initial calibration verification immediately after calibration. The performance of an instrument measurement system during an analytical sequence shall be verified by a continuing calibration verification.

The manufacturer's specifications for tuning the gas chromatograph/mass spectrometer (GC/MS) system shall be met before calibration. All systems incorporating a GC should have the retention time window specifications evaluated each time the GC system parameters are changed and whenever a new column is installed.

Continuing calibration should be verified routinely or before running samples. The responsible chemist is required notify the facility quality assurance officer and to take corrective action when organic measurement systems fail calibration.

\subsubsection{Physical Testing}

Minimum calibration requirements for several physical tests are presented in Volume 4, Section 4 , of HASQARD. 


\subsection{LABORATORY PROCEDURES}

Laboratory analyses and activities are directed and controlled by approved procedures. Procedures are approved before use in accordance with HNF-PRO-233, Review and Approval of | Documents, WMH-310, Section 1.8, and HNF-IP-1128, Section 1.0, "Procedure for Processing Internal Procedures. Each procedure has a unique identification number based on the approved numbering system in WMFI-310, Section 1.8 .

\subsection{TECHNICAL AND ADMINISTRATIVE PROCEDURES}

Procedures are prepared by chemists or other technically qualified personnel utilizing WMH-310, Section 1.11, "Writing and Reviewing Procedures" and HNF-IP-1128, Section 1.0,

| "Procedure or Processing Internal Procedures." These procedures are reviewed by designated technical organizations or individuals for correctness. Reviewers document comments on a I Procedure Review and Approval Form (PRAF) or an engineering change request for the internal procedures manual, HNF-IP-1128.

LA-approved procedures are issued as performance "goldenrod" copies with a unique identification (e.g., document number, revision/modification number), release date, and official release stamp. Electronic and hard-copy procedure distribution is completed by the Technical Support organization. HNF-IP-1128 procedures are controlled and released through the document control system and updates are sent to personnel on the distribution list.

| Other approved procedures include using vendor-supplied -manuals $r$, test plans, and desk instructions for performing analytical work. The use of manuals, test procedures, and test plans shall be documented and agreed by SAS and the customer. Adequate quality control shall be included to ensure that the precision, accuracy, sensitivity, and associated limitations (for example, detection limits) of the methodology are well understood (that is, method qualification) before reporting analysis.

Notebook test plans may be used to direct work for one-time and non-routine tests and procedures. These test plans shall be documented in controlled notebooks according to the requirements agreed to by the customer. As a minimum, peer review on the test or results shall be performed. Other approvals and signatures will be obtained at the discretion of the chemist and his/her management.

Procedures that produce data must have a "Q" approval code and include a quality control section. Procedures are reviewed as indicated by their approval designator at a frequency of 2,3 , and 5 years.

\section{Preparation and Review of Supporting Documents}

Supporting documents (SD) are used to document QAPPs, QAPjPs, basic laboratory practices, technical project plans, and laboratory test plans. Laboratory SDs do not have a specific format, but must have an identification number, a revision number, and a page number on each page. 
These documents provide a combination of administrative guidance, technical direction, and quality requirements. They are reviewed internally and externally based on the topic and application. The Supporting Document Release Station assigns identification numbers for all SDs.

The responsible manager for each SD identifies the reviewers within the WMH and PMHC organizations. The approval of the SD is documented by the reviewers on the engineering data transmittal (EDT), form BD-7400-127. An SD can be canceled and recalled by use of an internal letter.

\section{| 9.2 DOCUMENT CONTROL AND +CHANGE CONTROL}

Document control includes measures by which documentation can be controlled, tracked, and updated in a timely manner to ensure applicability and correctness. Control measures shall be used to ensure that documents are reviewed for adequacy, approved for release by authorized personnel, and distributed to and used at the location of the prescribed activity.

\subsubsection{Supporting Document Changes}

An SD can be revised by the author using an engineering change notice (ECN). Two types of ECN changes can be made: direct changes or supplemental changes. For direct changes, the ECN summarizes the change description and is the authorization for a new revision of the document to be issued. For supplemental changes, the ECN delineates the change details and becomes a part of the current document. Supplemental ECNs are incorporated into the document in the next revision. Refer to HNF-PRO-440, Engineering Document Change Control Requirements. Minimum approvals are by the approval designator, the author, and the author's manager. Supporting Document Release Station release-stamps the ECN and SD

\subsubsection{Procedural Changes}

The laboratory changes procedures (both regulatory and internally developed procedures) for a variety of reasons. The procedure change authorization (PCA) or PRAF is used to document and issue procedural changes for routine procedures and permanent changes. Three categories of changes are used based on the HASQARD; substitution, deviation, and modification. Procedure changes must be approved before use. If the PCA is a technical change and can affect reported data as the end product, QA must review and approve the change. Procedure change authorizations are distributed on pink paper. A substitution, deviation, or modification of a regulatory method (that is, CLP, SW-846) shall be documented in the appendix of the procedure.

9.2.2.1 Definition of Procedural Changes. Substitution is an adjustment in a procedure that has no significant effect on final results. This would be clearly evident in the QC data associated with the final results. 
Deviation is divergence from the original procedure that does not adversely affect the analyst's ability to meet the precision, accuracy, detection limit, selectivity, and quality control criteria of the procedure. Therefore, the decision to deviate shall be based on published literature (for example, alternative methods) and/or known sample chemistry.

Modification changes the character of a method; and, thereby, potentially limits a method's capability to meet the originally stated precision, accuracy, detection limit, selectivity, and quality control criteria. Because the impact of such a modification cannot be ascertained before implementation, it must be demonstrated by application.

9.2.2.2 Control of Procedural Change. Because substitution does not impactaffect the method performetused, no documentation of the change is required. Only the documentation necessary to allow reproducibility of results is required.

Deviation requires documenting the changes made to a procedure. Documentation of deviations made shall be included in the final report narrative. Justification of the deviation should be evident in the acceptable performance associated with the final results and also should be addressed. Acceptable performance shall be demonstrated by the analyst's ability to meet or exceed the original method's precision, accuracy, detection limit, selectivity, and quality control criteria. Whenever possible, the client should be notified of deviations before starting work. When a deviation is used routinely, it shall be incorporated into the procedure.

An example of a procedure deviation would be using different sample sizes accompanied by subsequent ratioed changes to all reagents and standard additions while maintaining the same final extract concentration. In some very limited cases, deviation might include varying reagent additions to effect similar digestion and/or analytical performance to the original procedure (for example, addition of matrix modifier). A deviation also may be an additional precipitation reaction resulting in enhanced analyte purification.

Modification requires the procedure to be qualified, documented, approved by laboratory management, and agreed tpon with the client before work. Requirements for implementation and personnel training shall apply, as necessary, to all laboratory procedures. Justification of the modification should be evident in the QC data associated with the final results and also should atso-be addressed. A modification with long-term applicability should be developed into a revised laboratory procedure.

Examples of procedure modification include using closed-vessel digestion instead of standard beaker digestion, using alternative reagents forbased on waste-disposal or safe-handling considerations, using different sample sizes accompanied by non-ratioed reagent addition, using alternative technology, and using extended holding times.

NOTE: WMH-310, Section 1.8, "Laboratory Procedure Process", part 7.0, relates to change control, review and approval changes, etc. 


\subsubsection{Procedure Cancellation}

A procedure can be made inactive or canceled from use by the technical authority or approval authority using the PRAF or other signed notification. Once approved, the procedure is tracked in the procedure database, included in the daily report, and pulled from the field. HNF-IP-1128 procedure cancellation is communicated via document control to the personnel on the distribution.

\subsection{NEW ANALYTICAL METHODS}

The EPA, DOE, and consensus methods (for example,ASTM], standard methods) are recommended where the technique is applicable to the sample matrix and the overall objective of. the analysis.

Procedures for new analytical methods are qualified before use. New methods are defined as methods used for the first time, whether based on published, well-understood procedures or developed in the laboratory. The user will document the method's performance using simple standard materials to establish accuracy and precision, and detection limits, and run interference studies to determine parameter variability effect of instrument parameters, as appropriate. The performance shall be verified using certified standards, matrix standards, spikes or method standard addition, sample exchange programs with other laboratories, and comparison with standard or accepted methods on actual samples, as appropriate.

\subsection{MODIFICATION OF REQUIRED REGULATORY METHODS}

Data Quality Objectives vary considerably between projects, and there will always be-situations will occur in whch the use of even grossly modified anlytical methods would be acceptable, based on the needs of the project. Modification changes the character of a procedure and thereby potentially limits a procedure's ability to meet the originally stated precision, accuracy, detection limit, selectivity, and QC. Examples of modifiation would include; using a closed-vessel digestion instead of standard-beaker digestion, using alternative analytical technology, and/or using extended holding times. It is important to emphasize that cases exist in which modifications are necessary and or appropriate. These include cases with the following characteristics.

- Published performance criteria cannot be duplicated using similar apparatus and operating conditions.

- The intent is to combine multiple, similar methods into a single method

The following procedures shall be used when modifying required regulatory methods. These procedures shall be followed only when the precision, accuracy, detection limits, and/or quality control criteria of approved methods might be affected either positively or negatively. 


\subsubsection{Justifying Modification}

The citation of the original, required regulatory method shall be provided. All modifications to the required regulatory method shall be specifically described by providing a synopsis (or direct quotation) of the regulatory method requirement and a description of all changes made. The reasons the requirement cannot be met and/or the technical, health and safety,

environmental, and/or waste disposal merits of the modification also shall be provided.

\subsubsection{Regulatory Notification}

The notification mechanism available to the laboratory requires $R L$ to coordinate with the regulator. The laboratory must obtain documented approval from RL to use the new procedure before starting work. The time frame for acceptance shall be documented and agreed upon. Information regarding regulatory acceptance considerations can be found in references such as WAC 173-303.

\subsubsection{Documenting the Modified Method}

In cases where changes are restricted to specific sections of the required regulatory method, the text of the modification shall be provided by the technical authority (for example, different instrument configuration, different spike, or surrogate compounds). A complete copy of the modified method shall be provided to Procedures Administration for processing a change. The modified method shall be managed by Procedures Administration as a controlled document, subject to the necessary review and approval. If the PCA is a technical change and can affect reported data as the end product, QA must review and approve the change.

The impact of the changes on the published precision, accuracy, and/or detection limit of the modified method shall be established by experiment. Any modification to the approved QC procedures for the method shall be described and the acceptance criteria speciffed (for example, using special surrogates and/or spikes, detection limit). See Section 8.5 for the approach required for method qualification.

Implementing the final modified method as a production method in the laboratory requires signatures of approval attesting that all requirements have been met. Approval signatures are required from the laboratory QA representative and a representative of laboratory management from the section where the method is to be used.

All original laboratory test data shall be retained on file to enable retrospective examination of the method should the need arise. 
HNF-SD-WM-QAPP-034 Rev. 3

\subsubsection{Reporting Results from Modified Regulatory Methods}

All modified methods shall be issued with unique identification codes to notify the data user that the method has been modified. To the extent practical, modified methods shall retain a method reference (identifier) to the original method.

\subsubsection{Acceptance Criteria for Modified Methods}

Modified methods shall include the acceptance and performance criteria for precision, accuracy, calibration, and detection limit established during the qualification experiments.

\subsection{QUALIFICATION OF ANALYTICAL METHODS}

Qualification is the process of determining the suitability of a preparative or analytical measurement system for providing useful analytical data. Performance parameters of the method are compared with the requirements for the analytical data. Several approaches may be used to qualify a method and include the following:

- When suitable reference materials are available to adequately test method performance versus matrix effect, performance can be demonstrated quite easily. This test consists of analyzing a sufficient number of reference samples and comparing the results obtained to those quoted for the particular material. A simulated matrix may be the closest performance indicator available.

- When suitable reference materials are not available, two other approaches are considered reasonable. The first involves comparing the new method against a known, well-established (laboratory-approved or regulator-recognized) method; the second involves inter-laboratory comparisons. In limited cases, matrix spikes and/or surrogates may be used; this is the least desirable because of the limitations associated with preparing spike and/or surrogate materials. Also, spikes and/or surrogates may behave differently from the actual sample in the process investigated.

Generally accepted standards dictate using a minimum of four replicates for each test case. Whenever possible, seven replicates should be used. These data should-be used to establish statistical control on an advisory basis until sufficient data (typically 30 data sets) are acquired-:

A method also must be evaluated for its overall effectiveness in the areas of sensitivity, linear range limitations, matrix or analytical precision, accuracy, and counting statistics (radiochemistry), as applicable to the method and/or analyte. Method testing includes the following:

- Method detection level determination and/or minimum detectable activity

- Method blank evaluation 
HNF-SD-WM-QAPP-034 Rev. 3

- Precision and accuracy determination

- Counter performance, if applicable

- Uncertainty

- Determination of method interferences as appropriate to the method (i.e., preparative versus determinative). 
HNF-SD-WM-QAPP-034 Rev. 3

This page intentionally left blank. 


\subsection{DATA COLLECTION, REDUCTION, AND REPORTING}

Data collecting and reporting processes include proper sampling, correct chain-of-custody practices, collection of raw data, data reduction and calculations, and transferring results to a final form for reporting. Data storage must meet specified records inventory disposal system (RIDS) requirements.

\subsection{DATA COLLECTION}

Raw data can be generated manually or electronically. Manual collection is done by the analyst and recorded on laboratory work sheets, batch pages, or log books according to applicable procedures. Data reported by the laboratory must be gathered using approved controlled procedures. "Raw data" is defined as data that cannot be easily derived or recalculated from other information and is referred to as the "quality record." Raw data are collected and maintained based on the method or instrument. For instruments with a built-in integration or detector signal processing software, the instrument controller output is considered the raw data. Raw data must be signed and dated by the chemist that performed the analysis. Only one signature per analytical run is required, provided the run generated can be traced back to the analyst. Signatures should be legible; if not, the name of the analyst/chemist must be hand-printed near the signature.

Information used in sample preparation (for example, weight or volume of sample used, percent dry weight for solids, dilution factor used) and in the calculations (for example, raw data or detector signal data, calibration, tuning records, interference check results or correction factors, blank or background correction values) shall be maintained-in order to enable reconstruction of the final results at a later date.

The cognizant chemist is responsible for designating a raw-data filing system that complies with documented RDDS requirements, if the raw data are not submitted to the $\mathrm{PC}$ for addition into a data package. Raw data can be filed by project; or batch, or can be filed chronologically. Raw data isare controlled by the laboratory and may be reviewed by the client upon request. Managers shall perform self-assessments of record inventories to ensure that they comply with RIDS requirements.

Each individual generating data or information is responsible for identifying data entry errors, sample identification errors, and calculation errors. The person entering the raw data is responsible for checking the integrity between raw data and entered data. Data must be legible, understandable, and reproducible with a standard photocopier. Reproduced copies must meet the " $3 X$ rule", where the third generation copy produced must be legible. Sample and QC results are calculated from raw data, then either manually or electronically entered into a spreadsheet for the project data package or report. 
Data entry errors shall be corrected by drawing one line through the error, then dating and initialing the line. Data changes shall be marked by drawing one line -through the change, then dating, initialing and explaining the change, as appropriate.

Data records are dispositioned according to the laboratory RIDS (See Section 10.0).

\subsection{DATA REDUCTION}

| Data from sample analysis should be reduced following applicable procedures. Data reduction includes activities that convert analytical measurements and instrument responses into reportable results. These activities may involve calculations changing the units or the data values, or conducting statistical, and mathematical analysis.

The following practices shall be in place to ensure accuracy of data entry, proper calculation, and appropriate data reduction:

- Verify that all readings or output are accurate.

- Ensure proper error correction or data change, that is, drawing one line through, dating, initialing, and explaining, as appropriate.

- Select appropriate formulas for calculating final results, correct for appropriate backgrounds and/or interference (for example, Compton effects for gamma energy analysis [GEA] and inter-element correction for ICP), and document calculations and results.

- Verify that data are accurately transcribed into notebooks, forms/bench sheets, or spreadsheets.

Calculations used for generating a final result are described in the procedures. The chemist is responsible for calculating and checking the accuracy of the final results. The quality assurance officer is responsible for randomly checking calculations. Quality control calculation checks are performed during the data package review process.

Computer programs or spreadsheets used for data reduction shall be verified with known results before use to ensure calculation and data manipulation programs perform properly.

\subsubsection{Significant Figures}

Significant figures reflect the limits of the particular analysis method or measurement system. The contributing factor (instrument, constant or standard value) with the least number of significant digits defines an equal number of significant digits in the final result. Reporting analytical results to the appropriate number of significant figures areis determined by the responsible scientist.

Basic rules for significant figures and for calculating values and retaining the number of significant 
figures are provided in the referenced Standard Practice for Using Significant Digits in Test Data to Determine Conformance with Specifications, ASTM E-29 (ASTM E-29 1980, supplied by HASQARD). Reported values should only contain significant figures and the exponential notation should be used.

Significant figures are defined by the following rules.

1. All nonzero digits are significant (for example, 5.4337).

2. Zeroes between nonzero digits are significant (for example, 5403).

3. Zeroes to the left of nonzero digits are not significant but indicate the position of the decimal point (for example, 0.00345).

4. When a number ends in zeroes, the zeroes may or may not be significant depending on the source or measurement system that produced the number (for example, $1,000)$.

Vendor-supplied software may not meet the general rules for significant figures, and this would be acceptable; the laboratory should work with the client to determine the best way to report results, based on the project needs. Once the number of significant figures obtainable from a type of analysis is established, data resulting from such analyses are reduced according to set rules for rounding off (see Section 9.2.2).

\subsubsection{Rounding-Off Methods}

The rounding-off method is used when an observed or calculated value needs to be reported in a limited number of significant figures for determining conformance with specifications. Numbers shall be rounded off numbers at the end of a series of calculations. When a figure is to be rounded to fewer digits than the total number available, the rounding off procedure described referenced in ASTM E-29 (ASTM E-29, 1980 supplied by HASQARD) should be followed. Vendor-supplied software may not meet the general rules for rounding because of possible programming differences with the vendor-supplied software. The software rounding is acceptable.

The ASTM rounding rules can be summarized as follows:

1. When the figure next beyond the last place to be retained is less than 5 , retain unchanged the figure in the last place to be retained.

2. When the figure next beyond the last place to be retained is greater than 5 , increase by 1 the figure in the last place retained.

3. When the figure next beyond the last place to be retained is 5 , and no figures or only zeros are beyond this 5 , increase by 1 the figure in the last place retained if it is odd, leave the figure unchanged if it is even. Increase by 1 the figure in the last place retained, if figures are beyond this 5 . 


\subsection{DATA REVIEW}

Data review refers to the process of determining whether data conform to specified requirements: Customer QC requirements will not dictate method controls such as calibration, blanks I (contamination evaluation), or standards (statistical process control limits). Sample-quality tests, such as duplicates and spikes, are evaluated against the customer's TSAP, SAP, or other work authorizing documents with considerations for factors such as detection limits, spike levels, and sample heterogeneity. Where modifications are requested, clear written instructions must be received from the customer with laboratory concurrence before submission of samples. The data I review system is described in the following paragraphs in accordance with laboratory-established procedures to review data before data reports are prepared.

The chemists; or team lead can perform data entry and calculate results and/or QC parameters (precision or accuracy). The data are then reviewed $f$ or verified by the responsible chemist. The technical manager/lead/supervisor or chemist performs a peer reviewtand approves approvat and forwards the data package to the project lead. The laboratory $Q A / Q C$ staff will perform an independent review of the compiled data package. The data review activities are summarized as follows.

- The person who is responsible for manual data calculations shall check calculations against laboratory procedures.

- The chemist is responsible for reviewing data against applicable QC criteria to verify that the analytical system is performing acceptably (see-Chapter 11). If $\mathrm{QC}$ data does not meet $\mathrm{QC}$ requirements, data within the batch shall be evaluated to determine if the data were adversely affected; the sample shall be rerun, or the data shall be reported with qualifier(s), which will be detailed in the narrative as appropriate to the condition.

- The manager/lead or chemist (peer review/approval) is responsible for checking the calculations performed by the analyst, checking calibration information to ensure the stability and accuracy of the instrument, and checking administrative records or documents to ensure the calculated results are associated with the appropriate samples.

- Sample identification during sample collection and preparation; and certain raw data records are transferred manually and are checked by the analyst, chemist, and/or by the manager.

- The quality assurance officer is responsible for performing independent spot checks on QC parameters, calculations, administrative information, and for checking that data are accurately transcribed. The frequency of data review by the quality assurance officer depends on the type of project, ranging from 10 percent to 100 percent. This review is performed in accordance with WMH-310, Section 8.2 . 
- The project coordinator, team lead, or manager is responsible for checking compliance with the request for analysis, from the TSAP, memorandum of understanding (MOU), letter of instruction (LOI), or other customer requirements.

Errors detected in the review process shall be referred to the appropriate responsible party for corrective action.

\subsection{DATA REPORTING}

Measured parameters, the details of analysis, and the data values are reported in accordance with the requirements of the end-user as specified in the agreement between the laboratory and the client. The type of information, level of approval, data reporting format, and means of delivery shall be discussed and agreed tpon between the laboratory and the client (see Section 9.4.2 for information required in the reporting documentation). The inorganic or organic results are reported as numeric values. If the value is less than the instrument detection limit (IDL) or method detection limit (MDL), it is reported as the numerical detection limit proceeded by a less than symbol $(<)$. If the detection limit is reported elsewhere, the results may be reported as undetectable (for example, "ND").

Appropriate data qualifiers are either used with the results or discussed in the case narrative to provide information on the confidence level of the results.

SAS provides various types of data report deliverables ranging from simple data summary reports to a completed data package. In addition, telephone reporting is acceptable for emergency situations and shall be agreed trpon between the laboratory and the client.

| The original data package reporting process is described in WMH-310, Section 9.2, "Laboratory Report Generation".

| Chemists are responsible for documenting comments or concerns, where applicable, so the team lead can relay this information to the client.

When a review is completed by the chemist and necessary corrections are made, the analytical batches are transferred to project coordinators. The data are assimilated and the team lead writes the case narrative in the data package format required by the specific project plan. The quality assurance officer must be notified if any changes are made after the document QA approval date. The quality assurance officer primarily reviews the calculations for accuracy; and precision, and performs QC checks against the established QC criteria. Those items found deficient or requiring further explanation are recorded onto comment forms, requiring timely response from the responsible individual.

Management and or the team lead is responsible for customer communication of laboratory issues, entering the appropriate request for analyses (only the analytest and compounds requested), reporting data to the customer, and forpreparing data packages. 
The team lead is responsible for collecting the chemist comments or concerns from worklists and incorporating them into the project narrative (data deliverable case narrative). The team lead is also responsible for coordinating the generation of the data report. The data report is compiled in accordance with administrative procedure WMH-310, Section 9.2, and WMH-310, Section 2.8, "SAS Data Reporting, Verification and Review."

\subsubsection{Data Reporting Formats}

The results of the analyses required in the work authorizing document shall be documented using the following reporting formats. Each sampling and analysis event could include data reported using several of these formats. The specific reporting format categories shall be identified in each work-authorizing document. All data reports must be:

- Legible

- Clearly labeled as toby project (that is, tank, core, auger, grab) and date of issue - Paginated (Formats III through VI only).

10.4.1.1 Immediate Notification Reporting. If the program or sampling activity requires immediate notification of a result that exceeds an established safety or operational limit, the laboratory shall call the appropriate manager immediately upon the first review of the laboratory data by the responsible individual. This verbal notification must be followed be electronic notification to the manager. The work-authorizing document shall designate who shall be notified. Management and or the team lead is responsible for communicating the safety or operational limits to the laboratory personnel.

This type of reporting does not require any supporting raw data, QC information, or documentation of analytical procedure numbers used. The work-authorizing document shall clearly identify the action limits for each parameter, the method of notification, and-any other follow-up activity that may be required.

NOTE: It is recommended and advised that $\mathrm{QC}$ personnel review the report before release.

\subsubsection{Waste Management Data Package Reporting}

This type of reporting is designed for projects where the data are not intended to support regulatory compliance at the present time, but may be needed later for regulatory purposes. The objective of this data package is to collect the information needed from characterization verify or reproduce the reported results at a later date. This type of report also may be desirable for reporting data that are expected to be reviewed extensively by outside organizations. Because the regulatory or end use of the data may not be completely defined at the time of the analysis, no third-party validation of the data is required. Therefore, this report resembles a regulatory data package without third-party validation. The data package should be prepared by tank and include the data from all core, grab, or auger samples, including composites, segments, subsegments, drainable liquids, and associated blanks taken and analyzed from the tank during a single sampling 
activity. The data package should be organized into a summary report section and a data report section with appendices as applicable.

This data package shall be issued as a document approved for public release through the document control system. The raw data shall be accessible to the program until the respective waste tank is closed or the waste is treated.

10.4.1.3 Legally Defensible Data Package-RCRA Data Package. This data package is sometimes referred to as a "stand-alone data package" and is intended to support projects and sampling activities where the data isare used to meet regulatory compliance areas and could be subject to litigation. The data package has many of the same requirements for data summaries and narratives as a waste management data package but also requires that the data be validatable and stand-alone using validation procedures. In addition the data package shall include the following information:

- Laboratory control sample/standard concentrations and all raw data (including logbook pages) needed to check the calculation of the percent recovery

- All raw data needed to check the calculation of the reported blanks

- All raw data needed to check the RPDs and percent recoveries reported

- ICP-optical emission spectroscopy (OES) and ICP-mass spectrometer (MS) sensitivity and linear ranges

- Metal interference check sample results

- Initial and continuing calibration verification raw data

- Instrument tuning data and instrument run logs

- Column performance check (organic analyses) with standard including the chromatogram

- Chromatograms (for organic analyses)

- Sample identification

- Method identification

- Retention time of compound(s) identified

- Quantitative chromatogram report

- Analyte retention time

- Amount of sample injected

- Results of response factors

- Surrogate recovery results

- Concentration of compound found

- Date and time of injection 
HNF-SD-WM-QAPP-034 Rev. 3

- Calibration Data

- Calibration curve or empirical equation for the curve

- Correlation coefficient of the linear calibration

- Concentration and/or response factor data for calibration check standards including dates of analysis

- GC/MS daily tuning results

- Calibration data should be submitted by the chemist to the data packaging group as requested.

10.4.1.4 Special Data Reports. Data reporting requirements that do not fall into these categories or that take exception to some of the requirements in these categories may be implemented by incorporating a modified format for reporting to the work-authorizing document.

\subsubsection{Data Reporting Documentation}

These report documentation requirements follow TWRS MOU requirements, ISO/IEC General Requirements for the Competence of Calibration and Testing Laboratories, National Environmental Laboratory Accreditation Program (NELAP), American Petroleum Institute (API) and Good Laboratory Practices (GLP).

The reporting documentation should include the following information.

\section{Summary Report Section}

- Report title

- Unique report identification number or SD number

- Laboratory name and address and phone number

- Client name and address and contact phone number

- Project name

- Clear identification of any revisions (if applicable)

- Signature and title of responsible party (typically laboratory manager)

- Statement to not reproduce the report without written approval from the 222-S Laboratory or the customer, unless approved for public release 


\section{- Table of contents}

\section{Case narrative}

c. Introduction. Include -a summary that either clearly states that no criteria were exceeded or identifies those parameters that exceeded the established criteria or action limits. List notification action taken, if required. Identify the project information such as tank, core, segments, subsegments auger, grab samples or subsamples. List hold times, such as $<48$ hours or unable to meet hold times, if applicable.

b. Description of the Samples. Briefly describe the sample's physical characteristics (color, homogeneity, texture). Identify any unusual properties of the sample, any problems associated with subsampling or preparation, and percent recovery. For example with core samples, the mass of recovered drainable liquid and the mass of recovered solids should. be provided.

a. Discussion of Analytical Results. Briefly describe the analytical methods used (for example, cyanide quantitation by titration or spectrophotometry) and any changes to the TSAP-referenced procedure that may have been necessary to analyze the samples. Any analytical method used that is different from the original method requested by the customer must be identified and documented that the change had no significant effect on the quality of the results reported (unless otherwise instructed by the TSAP or work-authorizing document). Reference the procedure number and revision in this section. An ECN is still required to reflect a procedure change that is different from the TSAP request (for example, The TCLP procedure requires 100 grams of sample. To address as-low-as-reasonably achievable (ALARA) policy issues, the sample size is reduced to $<100$ grams. Documented this on the batch narrative, then carry it to the case narrative. Issues such as the example given should be discussed with the customer before starting the analysis).

In addition, include the following items in the Analytical Results discussion section (Part c):

- Briefly describe digestion/dissolution, preparation/separation, or extraction, and analytical methods used.

- Identify ifwhether any "immediate notification reporting" was necessary. List what laboratory and customer sample ID numbers were involved, who was notified, and when and how the information was communicated:

- Identify any QC or analytical problems (that is, precision accuracy, sensitivity) encountered during the analysis referenced to the affected batch, that may impact the results and their use for making safety, operational, or other decisions. 
- Discussion of any observations or possible causes that impact the overall quality of the analytical results (that is, sample integrity).

- Describe any activities (reruns, replicate or triplicate analyses, procedure modifications) that may have been used to verify the data. Describe coding on summary table such as " $R$ " on the right-side of the Sample \# column that denotes replicate results listed as " 1 ," and A \# column that denotes sample preparation aspects (that is, $\mathrm{F}=$ fusion and $\mathrm{A}=$ acid).

- Describe any assumptions or corrections applied to the data, such as use of standard additions or calculations that may be important to interpreting the data.

- Identify any samples not analyzed or analyses required by the respective work-authorizing document that were not performed, and on what sample each missing analysis was to be run.

- Tabulate any triplicate analyses.

- List any reruns results.

- Include sample information, including unique laboratory identifier cross-referenced to client identification, sample collection date and time, date of sample receipt, and date(s) of sample preparation and analysis

- Report analytical units and results with appropriate number of significant figures

- Identify any amended test results; signature and title of person accepting responsibility for the report contents; and subcontracted results, if applicable

- Include appropriate QC results (correlation with sample batch shall be traceable and documented)

- Include appropriate data qualifiers with definitions and a narrative on the quality of the results, if applicable.

d. Chain-of-custody documentation

e. Unique reporting issues (refer to Data Summary section).

f. The summary report tables shall only include qualified and verified data based on batch quality control as defined by the method. HASQARD requires all data reported shall have appropriate qualifiers $(\mathrm{QC}$ verification). Opportunistic analyses do not meet this requirement. Opportunistic data are not verified by the chemist and may not meet QC requirements. Analyses on opportunistic analyses also violates ALARA principlesand unnecessarily subjects laboratory personnel to additional 
radiation exposure (for example, sample handling) when reruns are not necessary. Only the requested analytes shall be tested for. The team lead is responsible for communicating customer-required analyses to the laboratory.

g. References. Any references (for example, SAS QAPP, TSAP, LOI, MOU, ECNs or extrusion logbooks used in the hot cell) should be listed in this section.

10.4.2.1 Data Summary. The data summaries may be presented in different formats depending on the type of analysis and the customer's needs. The QC results that should be reported are those needed to evaluate the sample results (duplicates, spikes, laboratory control samples (standards), preparation blanks). The following information is considered important to the data summaries for most chemical and radiochemical measurements:

- Sample identification

- Laboratory control sample/standard including percent recovery

- Preparation blanks, including identity and concentration of each constituent identified

- Sample, duplicate (including RPD), and replicate results

- Results of matrix spikes, pre- or post-digestion spikes and tracers or carriers. (see raw data, Section c)

- Surrogate analyses (GC/MS), GC, and high-performance liquid chromatography (HPLC) analyses (see raw data, Section d)

- Internal standard results

- Detection limits

- Report uncertainty/counting error for radiochemical analysis

- Additional data reporting (that is, the percent of moisture/solid or correction for equivalent dry weight may be included if requested by the client)

- Unique reporting format issues and data summary tables shall be identified in the case narrative (that is, action limits where only one entity is applicable)

10.4.2.2 Raw Data Section. Raw data for each type of measurement will vary depending on the activity or the analytical instrumentation. The raw data will be used to confirm that the resultsof 
sample and QC analyses were performed and the results calculated properly and the analytical measurement system was in control while the data were being generated.

Examples of the raw data would be:

- IC chromatograms by analyte

- ICP integration data by analyte

- Amount of spiked material must be documented in the raw data

- Amount of spiked surrogate must be documented in the raw data.

NOTE: Supporting Data. The record copy of the supporting data for the data package is retained by the laboratory unless specified differently in specific projects such as TRU Waste Characterization, it does not need to be included in the data package. Supporting data includes, but are not limited to, the following information:

- Results of standard additions

- Results of serial dilutions

- All raw data necessary to check calculation of analyte calculation (for example, calibration data)

- Mass spectrum including spectra of standards (one for each report for each compound detected) and spectra of analytes detected

\subsubsection{Data Package Structure (Generic)}

This will be a generic structure and may be changed by the user if the request is achievable or within the time constraints given to the team lead. The following items are recommended:

- Title/cover page

- Signature/approval page

- Table of contents

- Case narrative

- Custody documentation

- Data summary tables

- Raw data (if applicable)

- $\quad$ Supporting data (if applicable)

- Attachments.

\subsubsection{Preliminary Reporting}

A preliminary data reporting system shall be established between the client and the laboratory to address emergency situations. The type of information, level of approval, data reporting format, and means of delivery shall be discussed and agreed upon between the laboratory and the client. 
HNF-SD-WM-QAPP-034 Rev. 3

The emergency situation may include, but is not limited to, screening activities for safety issues, critical analytes, or limiting sample amount. 
HNF-SD-WM-QAPP-034 Rev. 3

This page intentionally left blank.

$10-14$ 
HNF-SD-WM-QAPP-034 Rev. 3

\subsection{RECORDS}

Procedures HNF-PRO-208, -210 through $-216,-222$ through -233 documents the requirements for preparing, reviewing, approving, issuing, using, and revising documents that prescribe work processes and specify requirements (refer to Chapter 8 for procedure document control information). Also, the applicable HNF procedures address the issues of identifying, preparing, approving, transmitting, correcting, distributing, retaining, and disposing of quality records.

This section is a short overview of requirements that apply to records that document the completion of work, such as laboratory analyses or training that support technical and regulatory decisions; or that provide objective evidence of conformance of an item, service, or process to regulatory, technical, or administrative requirements.

Laboratory quality assurance records include, but are not limited to the following:

- Analytical data (that is, raw data and supporting data)

- Electronic instrument files

- Certificates of reference material (standard traceability)

- Logbooks

- Procurement documents

- Training records

- Calibration records

- Maintenance records

1. - Chain-of-custody forms.

A quality assurance record is information contained on legible hard copy (paper), microfilm (with proper authorization), magnetic tape (electronic systems), or photocopies that are complete in terms of appropriate content and furnishes evidence of the quality of items, and/or activities assuring quality. Records may be original documents or legible reproduced copies. Other QA records may be required, such as those listed in HASQARD, Revision 1, Volume 1, Section 5.3, and Volume 4, Section 3.3.

A quality assurance record is classified as a "lifetime record" if it meets one of the following criteria:

- It documents performance (analysis) of hazardous waste site studies, monitoring, and site remediation or tank characterization projects.

- It would be of significant value in demonstrating capability for safe operation.

- It would be of significant value in maintaining, reworking, repairing, replacing, or modifying an item.

- It would be of significant value in determining the cause of an accident or malfunction. 
- It would provide required baseline data for in-service inspections.

- It would be used to identify and assess the performance capabilities of the engineered and natural barriers important to waste isolation or disposal.

- It would be required for substantiating development of major decisions involving questions of design safety, environmental protection, or program direction.

- It is associated with hazardous material packaging and transportation.

It is a record of repository licensing or environmental permitting.

Nonpermanent QA records are those required to show evidence that an activity was performed in accordance with the applicable requirements, but that need not be retained for the life of that item because they do not meet the criteria of lifetime records.

Post-closure QA records are those retained for 300 to 1,000 years, depending on the lifetime of the specific waste package. Post-closure records are typically site characterization documents of nuclear waste repositories.

Lockheed Martin Services, Inc. (LMSI), is responsible for the quality records system based on HNF-PRO-215 and HNF-PRO-222, which govern specifications, preparation, review, approval, and maintenance of quality records. A computer system is used by the Performance Measurement Team or LSMI to maintain and access quality records including electronic media to ensure the records are usable and retrievable. This computer system provides information on "inactive" records only; that is, records that have been transferred to the records holding area by the laboratory. "Active" records maintained in the field will not be retrievable through this computer system.

Instructions, procedures, and drawings shall be reviewed and approved by appropriately qualified individuals. Revisions to instructions, procedures, and drawings that affect the process or are technical in nature shall receive the same level of review and approval as the original document. Editorial changes may be made to instructions, procedures, and drawings without review and approval. Refer toChapter 8 for additional information about procedure document control requirements.

\subsection{GENERATION OF QUALITY RECORDS}

Hard-copy documents and data referenced by final reports shall be retrievable from the records system, except for readily available references (for example, national codes and standards). Appropriate materials and recording techniques shall be used to ensure the preservation of records as long as they are needed.

Sufficient records shall be specified, prepared, reviewed, authorized, and maintained to reflect the achievement of the required quality. Records shall include such documents as operating logs, 
review results, inspections, tests, assessments, work performance monitoring, material/sample analyses, and calibration records.

Records also shall include personnel qualifications, procedures, and equipment. Inspection and test records shall include, at a minimum, the identification of the inspector or data recorder, the type of observation, the results, the acceptability and the action taken to correct deficiencies noted.

Maintenance of active records shall include provisions for transmittal, distribution, retention, protection, preservation, traceability, disposition, and retrievability.

Records shall be classified, retained, and dispositioned in accordance with DOE Order 1324.5B, Records Management Program (DOE 1987).

\section{The Hanford Site remains under a full moratorium on the destruction of all record material.}

\subsection{RECETPT CONTROL}

The Performance Measurement Team is responsible for maintaining the Laboratory Technical Information Center and for coordinating retrieval of the record copies for information. LMSI is responsible for receiving the records and implementing a system of receipt control of the records for permanent storage. LMSI has the capability to do the following:

- Designate the required records

- Identify records received

- Receive and inspect incoming records

- Submit completed records to the records-holding facility without unnecessary delay.

\subsection{RECORDS VALIDATION}

Records considered valid shall be signed, initialed, stamped, or otherwise authenticated, and dated by the document's originator (HNF-PRO-222). Hard (paper) copies of electronic records shall be validated by signing and dating a paper copy of the first page of the record by the document's originator. These records may be originals or copies. Handwritten signatures are not required if the record is clearly traceable to the person or organization who created the record. 


\subsection{CORRECTING RECORDS}

Records are to be corrected as described in HNF-PRO-222, Section 4.4. The correction or - revision of technical or quality-related information shall have an appropriate review and approval by an authorized person of the originating organization responsible for approving the in-process document. The name of the person authorized to issue the correction and the date of correction shall be marked on the quality record.

Correction of in-process documents shall be accomplished by striking a single line through the incorrect information and inserting the correction as close as possible to the original data. The corrections shall be initialed and dated by the person making the correction. If a single page contains multiple corrections, each must be initialed and dated by the person making the corrections.

NOTE: If pages in an in-process document contain erasures, white out, correction tape, or other marks that make the in-process document unacceptable as a complete record, the affected pagefst shall be regenerated or photocopied and submitted as the record.

Complete records that have been transferred to a designated storage location shall be corrected by supplementing the record with the correct information (see HNF-PRO-215. Complete records that have not been transferred can be corrected in the same manner as an in-process document.

\subsection{RECORDS IDENTIFICATION AND INDEXING}

I Records are cataloged and tracked on a database and can be retrieved through a variety of search fields including project or activity identification. LMSI is responsible for receiving, indexing, storing, maintaining, retrieving, and controlling records. Record retention times are controlled by the RIDS system. Location of the record is indexed on the Record Holding Area-Management Information System (RHA-MIS).

\subsection{MAINTENANCE AND RETENTION OF RECORDS}

FNF-PRO-215 and HNF-PRO-222 describes the maintenance of active records for transmittal, distribution, retention, protection, preservation, traceability, accountability, disposition, and retrievability.

I Chapter 11 and HNF-PRO-222 describes the criteria for lifetime records and nonpermanent records. The appropriate quality record retention time is monitored by the RIDS system. Expired records are destroyed. 
HNF-SD-WM-QAPP-034 Rev. 3

\subsection{RETRIEVAL AND DISPOSITION}

Records are retrieved from records storage facilities in accordance with HNF-PRO-215.

Records are dispositioned in accordance with HNF-PRO-214.

\subsection{REPLACEMENT, RESTORATION, OR SUBSTITUTION OF RECORDS}

Lost or damaged records shall be replaced, restored, or substituted when applicable (HNF-PRO-222).

\subsection{RECORDS TURNOVER TO CLIENT}

Records turned over to the client are typically copies of the original records. Original records are kept at the Hanford Nuclear Facility or other designated storage facility and controlled according to the appropriate record requirements defined in the applicable HNF procedure. 
Table 11-1. Recommended Report Deliverables.

\begin{tabular}{||l|l|}
\hline \multicolumn{1}{|c|}{ Data Package Item } & \multicolumn{1}{|c|}{ Contents } \\
\hline Case narrative & $\begin{array}{l}\text { Narrative, requested analysis,data and QC } \\
\text { summary tables, chain of custody, supplemental } \\
\text { information }\end{array}$ \\
\hline Sample results & $\begin{array}{l}\text { Bench sheets (sample preparation) } \\
\text { Printed results } \\
\text { Date of analysis } \\
\text { Instrument or analysis identification }\end{array}$ \\
\hline Calibration data & $\begin{array}{l}\text { Calibration dates and time } \\
\text { Raw data } \\
\text { Standards traceability } \\
\text { Control charts, where requested } \\
\text { Detector efficiency }\end{array}$ \\
\hline Blanks & $\begin{array}{l}\text { Blank identification } \\
\text { Date of analysis } \\
\text { Blank results } \\
\text { Preparation data }\end{array}$ \\
\hline Duplicates & $\begin{array}{l}\text { Duplicate sample identification } \\
\text { Date of analysis } \\
\text { Primary and duplicate percent recoveries } \\
\text { Preparation data }\end{array}$ \\
\hline Laboratory control & $\begin{array}{l}\text { LCS sample identification } \\
\text { Date of analysis } \\
\text { Results, limits } \\
\text { Preparation. data } \\
\text { Control charts }\end{array}$ \\
\hline Samples & $\begin{array}{l}\text { Amounts of spike added } \\
\text { Traceability of spikes } \\
\text { Recovery results and limits } \\
\text { Raw data } \\
\text { Preparation data }\end{array}$ \\
\hline Other QC & $\begin{array}{l}\text { Any other QC required in the DQO, SOW, LOI, } \\
\text { etc. }\end{array}$ \\
\hline
\end{tabular}

NOTE: DQO process, statement of work, letters of instruction, etc., will govern the data deliverables for each project.

DQO = data quality objective

LCS = laboratory control sample

LOI $=$ letter of instruction

SOW = statement of work

$\mathrm{QC} \quad=$ quality control 
Table 11-2. SAS Level D Quality Control Order of Sample Run.

\begin{tabular}{|c|l||c|l||}
\hline $\begin{array}{c}\text { Sample } \\
\text { Number }\end{array}$ & Description & $\begin{array}{c}\text { Sample } \\
\text { Number }\end{array}$ & \multicolumn{1}{|c|}{ Description } \\
\hline 1 & ICV & 19 & Sample7 \\
\hline 2 & ICB & 20 & Sample8 \\
\hline 3 & CRII & 21 & Sample9 \\
\hline 4 & ICSAI & 22 & Sample10 \\
\hline 5 & ICSABI & 23 & CCV2 \\
\hline 6 & PB & 24 & CCB2 \\
\hline 7 & LCS(S or W) & 25 & Sample11 \\
\hline 8 & Sample1 & 26 & Sample12 \\
\hline 9 & Sample1D & 28 & Sample13 \\
\hline 10 & Sample1S & 29 & Sample14 \\
\hline 11 & CCV1 & 30 & Sample15 \\
\hline 12 & CCB1 & 31 & Sample16 \\
\hline 13 & Sample2 & 32 & ICSAF \\
\hline 14 & Sample2L & 33 & ICSABF \\
\hline 15 & Sample3 & 34 & CRIF \\
\hline 16 & Sample4 & 35 & CCV \\
\hline 17 & Sample5 & 36 & CCB \\
\hline 18 & Sample6 & & \\
\hline
\end{tabular}

Notes: These examples gure the highest level of quality control. Quality control requirements depend are dejeten customer specifications.
$\mathrm{CCB}$
$=$ Continuing calibration blank.
$\mathrm{CCV}$
$=$ Continuing calibration verification.
CRIF
$=$ Final Contract Required Detection Limit.
CRII
= Initial Contract Required Detection Limit.
ICB
= Initial calibration blank.
ICSABF
= Final Interference Check Sample, Interferents and analytes.
ICSABI
= Initial Interference Check Sample, Interferents and analytes.
ICSAF
= Final Interference Check Sample, Interferents.
ICSAI
= Initial Interference Check Sample, Interferents.
ICV
$=$ Initial calibration verification.
LCS
$=$ Laboratory control sample.
PB
$=$ Preparation blank. 
HNF-SD-WM-QAPP-034 Rev. 3

This page intentionally left blank. 


\subsection{QUALITY CONTROL}

The QC requirements of this document will be used for SAS analytical work (minimum required QC if not specified by the customer) except as modified by the customert or project-specific DQOs/data quality requirements (DQRs) and communicated to the laboratory in the projectspecific work-authorizing document. Where modifications are requested, clear written instructions must be received from the customer with laboratory concurrence before submission of samples. This chapter describes the (QC) measures used for analyses performed.

QC is defined as the overall system of activities that provides checks and balances to the quality of the analytical services. QC data are evaluated against the method and customer requirements to measure how well the data meets the customer's needs. The goal is to provide data of known quality that are adequate for the intended purpose. QC data allows the laboratory and customer to measure the degree of conformance against the requirements.

To assess the precision and accuracy of a reported result, QC checks are incorporated throughout the data collection process (for example, sample preparationf and separation, analysis, reporting). They-provide a measurement tool for evaluating the effectiveness of the process. QC checks provide information, about performance of the measurement system and environment, as well as matrix-related impacts on the measurement system. The information gained from evaluating QC performance can then be used to implement corrective actions or improve processes.

Each category of laboratory analysis, radiochemical, inorganic, and organic, has a separate set of QC requirements. Each field of chemistry, or category, is further subdivided into two parts, preparation/separation QC and measurement/counting QC. Basic laboratory QC operations, applicable to all disciplines, are presented in Section 11.1. Basic QC operations consist of controlling the quality of reagents, standards, and gases used in the laboratory.

The $\mathrm{QC}$ requirements presented are designed to enable both the laboratory and the customer to ascertain the quality of the data produced. The $\mathrm{QA} / \mathrm{QC}$ requirements of this document will be used for all analytical work except as modified to be less or more restrictive by the customert or project-specificDQOs and $\mathrm{DQRs}$ and communicated to the laboratory in the project specific SAP or work-authorizing document. These $\mathrm{QC}$ checks provide information on precision, accuracy, sensitivity, and overall reliability of the reported results. The laboratory is required to document and meet the $\mathrm{QC}$ requirements outlined in this section.

The degree of QC required for analysis is determined through negotiations with the customerclient. It is based on the end use of the data. However, it may not violate the specific QC protocols of EPA analytical methods and requirements of HASQARD as stated. Customer QC requirements will not dictate method controls such as calibration, blanks (contamination evaluation), or standards (statistical process control limits). Should the customer require QC criteria that are less restrictive than the current method control, it must be agreed on before analysis and documented in the case narrative. The team lead is responsible for communicating the customer QC requirements to the appropriate personnel. Sample quality tests such as duplicates and spikes are evaluated against the customer's SAP or other work-authorizing 
documents with considerations for factors such as detection limits, spike levels, and sample heterogeneity. Failure to meet the desired requirements may result in reruns or other corrective actions. When QC criteria cannot be achieved for the batch, they are identified and addressed in the case narrative as described in Chapter 9.

\subsection{GENERAL LABORATORY QUALITY CONTROL}

The QC described in this section represents the basic laboratory systems associated with analytical operations and applies to radiochemical, inorganic, and organic analytical procedures. The use of substandard reagents, standards, gases, materials, and equipment can result in less reliable or unreliable data. The laboratory will have a mechanism in place for demonstrating control over | those-sources that affect the precision, accuracy, and sensitivity of reported results. The minimum requirement would consist of monitoring analytical and preparative blanks for contamination from these sources

\subsubsection{Reagent Water}

Reagent water is prepared using reverse osmosis (RO) followed by ion exchange using a mixed-bed ion exchanger and final submicron filtering. Water used for some organic analyses may also be treated by ultraviolet (UV) oxidation. The quality of the water is measured by the following:

- A resistivity check using an in-line meter or indicator on the ion exchanger. A minimum resistivity of 10 megohm-cm at $25^{\circ} \mathrm{C}$ is required (the corresponding conductivity is $0.1 \mu \mathrm{S} / \mathrm{cm}$ at $25^{\circ} \mathrm{C}$ ), but at this level requires approval of the method scientist. If the resistivity is $\geq 18$ megohm, the water may be used for all applications requiring reagent water.

- Assessments of sample blank data are evaluated as batches are performed.

- WMH-310, Section 8.5, "Quality Control of the RO/DI Water System."

Documentation of the water quality data (resistivity and conductivity) form is kept in tabular format at each station. When the form is full, it is given to the QA officer for addition to the QA record file. Blank data are available from IC, ICP, and other chemistry areas.

\subsubsection{Compressed Gases/Reagents}

Percent purity levels necessary for quality analysis are listed in each analytical procedure. The quality of gases or reagents is monitored by the performance of the preparation blank. 


\subsubsection{Standards}

The acceptability of standards used in the preparation and analysis of customer samples shall be verified as defined HNF-IP-1128, Preparation and Validation of Standards. The requirements are listed in Section 7.3.

\subsubsection{Labware}

When affecting quality, and if available, only Class A glassware will be used for laboratory operations. Plasticware can be used for qualitative measurements where the volume used does not affect the data. Vials and glassware should be cleaned in accordance with EPA Method 600 when analyzing samples for low-level concentrations of contaminants to support environmental compliance objectives. Labware will be cleaned according to operating procedure LO-080-116, unless otherwise directed in the specific analytical procedures.

\subsubsection{Housekeeping}

Employees are responsible for keeping their work areas neat and orderly mamer. Housekeeping inspections are performed by the shift manager on night shift. Housekeeping in general is the responsibility of the room owner. Housekeeping is performed routinely in accordance with the following procedures:

- HNF-IP-1 128, Section 1.3, "Safety and Housekeeping Inspection Plan"

- LO-100-107, Cubicle Housekeeping and Waste Management.

\subsection{INORGANIC PREPARATION TECHNIQUES}

Preparation techniques are those operations used to prepare a sample for analysis. Examples include: digestion, dissolution, extraction, phase partitioning, and/or leaching orof a sample material. Separation and/or isolation techniques are preparative if performed before analysis to facilitate reliable analyte measurement. Simple dilution is not a sample preparation technique. Preparation techniques should be selected based on the client's data quality requirements.

Blank analysis should always follow standard analysis to identify any carry-over effects.

\subsubsection{Laboratory Control Sample or Blank Spike}

The LCS or blank spike is used to monitor the effectiveness of the sample preparation process. An LCS is a material similar in nature to the sample being processed, containing known concentrations of the analytefst of interest (for example, standard reference material). The LCS is subject to the same analytical processing (preparation, separation, analysis, and so forth) as the client samples. An LCS is performed once per preparation batch for ICP and AA, once per 
analytical batch for other methods. A blank spike is normally used when an appropriate LCS is unavailable. The blank spike is distilled or deionized (reagent) water spiked with the analyte(s) of interest.

Results from the analysis of the LCS or blank spike are compared to the analyte values known to be present in the sample. LCS or blank spike acceptability is demonstrated by target analytes being within 80 percent to 120 percent administrative limits or statistical process control (SPC) limits. SPC limits are statistically determined by multiple analyses over time. New methods will use administrative (vendor, regulatory, or past experience) limits until enough data can be gathered to set limits statistically.

When the acceptance criteria for the LCS has not been met for an analyte of interest, all samples in the preparation batch shall be evaluated, corrected, reanalyzed and/or reprepared for that analyte. Client criteria will not override LCS control limits, unless agreed tpon with the client before the analysis in accordance with the HASQARD, Volume 1, page 1-7.

\subsubsection{Matrix Spike}

A matrix spike is a client sample that has been spiked with the analytetst of interest and processed in the same manner as the sample. The matrix spike is used to monitor method performance in a specific sample matrix. Matrix spike results measure the accuracy of the measurement of the analyte of interest present in the client sample matrix.

When the sample concentration is unknown, spiking is typically performed at one of the following levels:

- Equivalent to the regulatory threshold

- Specified in the method

- One to five times the estimated quantitation limit (EQL).

Otherwise, spiking should be performed at a level equivalent to that of the sample or at least 25 percent of the sample. However, general EPA spiking guidelines state that spike recovery limits do not apply when sample concentration exceeds the spike concentration by a factor of 4 (EPA 4X Rule) or more (that is, sample concentration of $500 \mathrm{ppm}$; a valid spike concentration, added to the sample, would need to be between the minimum of $125 \mathrm{ppm}$ and a maximum of $2,000 \mathrm{ppm}$ ).

The following are some EPA guidelines for evaluating spike recoveries. If the spike recovery is $\rightarrow$ greater than 125 percent and the reported sample results are fless than the IDL, the data are acceptable for use. If the spike recovery is $\rightarrow$ greater than 125 percent or fless than 75 percent and the sample results are $>$ greater than the $I D L$, the data should be qualified as estimated.

Matrix spike control is demonstrated when target analytes are within established control limits. Control limits are established by one of the following: 
- Regulatory requirement

- Client via data quality requirements for a particular project or program

- Laboratory performance.

The recommended target level for matrix spike analysis is 75 percent to 125 percent recovery. | Control limits are not applicable to spikes that are less than $\leqslant 25$ percent of the analyte concentration in the sample. Spike recoveries outside this range will be flagged and explained in the case narrative, not necessarily generating an automatic reanalysis. Repreparation and/or reanalysis should be conducted as necessary based on the DQOs and DQRs. Minimum spike frequency will be 5 percent, one per matrix if fewer than 20 samples are analyzed per matrix, or as requested by client.

A matrix spike may not be applicable if the analyte concentration in the sample is very large where addition of large amounts of spikes are not practical because of solubility concerns. Other methods of evaluating method performance, such as serial dilution, post-digestion/post-dilution spike, may be used. Where a sample matrix cannot be spiked at the time of original preparation, a post digestion spike shall be performed (Section 11.5.8). The term "matrix spike" can be used only when the sample is spiked at original preparation.

For inorganic analysis, matrix spike control is demonstrated when target analytes are within established control limits. Control limits are established by one of the following:

- Regulatory requirement

- The client via data quality réquirements for a particular project or program

- Laboratory performance over time.

The recommended criterion for most inorganic analysis is recovery within 75 percent to 125 percent.

If the matrix spike recovery fails to meet the criterion, the batch results shall be investigated for sources of error. The goal is to ensure that limitations on the data caused by the sample matrix and represented by matrix spike performance, are discussed in the batch comment narrative and carried into the case narrative of the report to the client.

\subsubsection{Laboratory Sample Duplicate or Matrix Spike Duplicate}

Laboratory sample duplicates are two aliquots of the same sample (intralaboratory split)-taken through the entire sample preparation and analysis process. Laboratory duplicates are used to assess the precision of the preparation and analysis process in a client-specific matrix.

Matrix spike duplicates are two spiked aliquots of the same sample taken through the entire sample preparation and analysis process. Where the sample is not expected to contain reasonable concentrations of the analytes of interest (analyte concentrations greater than 10 times the instrument detection limit), duplicate sample results will not provide a reliable estimate of precision. In these cases, matrix spike duplicates are used to demonstrate analytical precision in 
the client sample. To the degree possible, the laboratory and the client should agree upon the use of sample duplicates versus matrix spike duplicates before starting the work.

Agreement between duplicates indicates reproducibility of the combined preparation/separation, and measurement process (precision).

I Disagreement can occur because of differences in analyte concentration within the sample matrix (non-homogeneity) that are not amenable to analyst control during the analytical process (for

1 example, isolated particles of lead in soil matrix that cannot be reliably sampled using standard laboratory subsampling techniques). Disagreement may arise if the method has poor applicability to the analyte/matrix system.

One set of laboratory duplicates (or MS/matrix spike duplicate [MSD]) may be required for each sample batch or matrix type. Precision is estimated by calculating the RPD of the duplicate analysis. On receipt of instructions from the client, additional replicates can be performed. These additional samples will increase the likelihood of detecting non-uniformly distributed analytes in

the client sample. It will also will allow for a more accurate estimate of the variability of the overall analytical performance on client-specific samples/matrices. When more than two replicates are used, precision is expressed in terms of relative standard deviation.

Typically, inorganic analyses include a sample and sample duplicate because a high probability exists that the majority of those analytefst of interest will be detected in the sample. The inorganic duplicate RPD acriterion normally is set at 20 percent; this criterion shall be applied only to analyte concentrations greater than 10 times the instrument detection limit (or method detection limit).

Duplicate (or matrix spike duplicate) results shall be reported to the client along with the calculated RPD. Duplicate results should be discussed in the case narrative. When client DQRs cannot be met, the client shall be notified; results shall either be accepted or new work scope (methodology) agreed on.

\subsection{INORGANIC ANALYTICAL TECHNIQUES}

Analytical techniques are operations that permit the measurement of a parameter of interest. Analysis is the measurement of some property for which an instrument response occurs that can be related to the concentration or amount of the substance of interest in a sample or in some material prepared from the sample. Selection of analytical technique should be based on client $\mathrm{DQRs}$. The $\mathrm{QC}$ requirements to follow are designed to verify and document proper instrument operation and to give the analyst information regarding the precision, accuracy, and sensitivity of the analytical process. 


\subsubsection{Analytical Run or Sequence}

An analytical run or sequence is defined as a group of samples analyzed together that may include one or more preparation batches. A prescribed number and type of QC samples that are analyzed in a prescribed order is associated with each analytical sequence. The analytical sequence is an important aspect of the analytical work performed because it allows the analyst and subsequent data reviewers toidentify trends in sample results or QC related to the order in which samples were analyzed. This may allow the elimination of unnecessary reanalysis by a QC failure (cross-contamination between samples) during an analytical sequence. Therefore, the order in which samples are analyzed will be traceable to the analytical sequence.

For ICP and AA analyses, the analytical sequence ends with a continuing calibration standard and blank. More than one batch of samples can be analyzed in an analytical sequence as long as continuing calibration control is maintained.

\subsubsection{Initial Calibration Verification}

The ICV analytical standard is used to confirm the accuracy of the calibration and the standards used for calibration. Acceptable performance of the ICV demonstrates that both the standards used and the instrument are functioning properly. The ICV is prepared from a source other than that used to prepare the calibration standards.

Most inorganic techniques are subject to routine, frequent recalibration. The ICV is required whenever the system is recalibrated. The ICV shall be run following calibration and before analysis of client samples.

Failure of the ICV indicates instrument and/or standard problems that must be evaluated and corrected before any client samples are processed for the analytes of interest.

\subsubsection{Continuing Calibration Verification}

The CCV analytical standard is used to monitor instrument stability over time. Acceptable performance demonstrates continued appropriateness of the calibration, indicating that the system is still in control. The CCV may be prepared from any reliable source and need not be nationally or internationally traceable. The ICV standard also may be used as the CCV.

Each inorganic analytical system shall include periodic checks on the stability of the instrument calibration. Failure indicates that the analytical system has drifted out of control and requires corrective action for the analytes of interest. All samples analyzed after the last acceptable CCV shall be reanalyzed. Reanalysis applies to specific analyte failure. In limited cases, isolated analyte failures may be tolerated if sample results still meet the client's quality requirements. Reporting results in such cases requires justification in the batch narrative that must be carried forward to the case narrative. 


\subsubsection{Initial and Continuing Calibration Blanks}

Initial and continuing calibration blanks monitor effects such as contamination and instrument response drift during routine analytical analyses. The initialt or continuing calibration blank; is prepared similarly to the standards, but is not subjected to preliminary sample preparation except for techniques in which the preparation is an integral part of the analysis. In these cases, the preparation blank or method blank can be considered the equivalent of the initial calibration blank (ICB) or continuing calibration blank (CCB). The generally accepted criterion for these blanks is that they be below the EQL for each analyte of interest. When an analyte exceeds this criterion, analysis shall be investigated for potential impact. Samples with concentrations or activities exceeding the blank contamination level by a factor of 20 or more can be reported unless client requirements dictate otherwise. Additionally, samples with concentrations below the detection limits can be reported. In all other cases, all samples analyzed since the last acceptable ICB or I CCB; that were determined to have been affected by the blank contamination; shall be analyzed for the specific analyte failure.

For inorganic compounds, each ICV shall be followed by an ICB and each subsequent CCV shall be followed by a CCB. This protocol indicates potential carry-over effects (carry-over of residual material from one sample to the next in the sequence).

\subsubsection{Internal Standards}

An internal standard is an analyte that is similar to the analytefs) of interest in terms of its analytical response, but is not normally expected to be found in the sample. Internal standards are added to every standard, blank, matrix spike, matrix spike duplicate, and sample before analysis. Internal standards are used as the basis for quantitation of the target analytes of interest.

Internal standards are routinely used in ICP/MS analysis, although they also may be appropriate to other types of analysis. Selecting appropriate internal standards shall be method and analyte-list specific because all results are normalized based on internal standard performance. Laboratory procedures shall specify internal standards used and associated acceptance criteria.

\subsubsection{Low-Level Standard}

The low-level standard is used to monitor instrument performance in the region at or near the $\mathrm{EQL}$ and is applied to inorganic systems to monitor sensitivity in the EQL region.

\subsubsection{Interference Check Standards}

Interference check standards are typically applied in ICP systems (for example, ICP and ICP/MS). The interference check normally consists of two standards. The first standard contains known concentrations of the major interfering elements that will provide an adequate test of interelement correction factors. The second standard contains both the major interference and the majority of 
other analytes tested. The major interference eis spiked into all the standards at significant concentrations that are expected to produce an interference effect. All other analytes are spiked at relatively low levels. Data from both standards, when corrected, should recover between 80 percent to 120 percent for all analytes tested or an interelement correction is considered inadequate. The first standard, containing only the major interference of concern, should produce I no analyte concentration with an absolute value exceeding the EQL. Instruments capable of showing negative results do not require the second standard that contains both interference and additional analytes tested.

\subsubsection{Analytical Spike}

An analytical spike is a material that is chemically similar to the analyte, but can be detected separately. It is, added to the sample just before analysis (that is, after preparation). It differs I from the matrix spike because the spike is added to the sample before sample preparation. The analytical spike is used to gauge instrument and sample matrix performance during the measurement process. Typically, a very small quantity of spike is added so no significant change occurs in sample volume or matrix effects. The concentration should be performed at one to five time the EQL if no analyte is expected or the concentration is unknown. Otherwise, spiking I should be performed at a level equal to that of the sample, or at least 25 percent of the sample. However, general EPA spiking guidelines state that spike recovery limits do not apply when sample concentration exceeds the spike concentration by a factor of four (EPA 4X Rule) or more. (For a sample concentration of $500 \mathrm{ppm}$; a valid spike concentration would need to be between the minimum of $125 \mathrm{ppm}$ and a maximum of $2,000 \mathrm{ppm}$.):

The following are some EPA guidelines for evaluating spike recoveries. If the spike recovery is $>$ more than 125 percent and the reported sample results are \&less than the IDL, the data isare acceptable for use. If the spike recovery is $>$ more than 125 percent or $<$ less than 75 percent and the sample results are $>$ more than the IDL, the data should be qualified as estimated.

Analytical spikes are required for graphite furnace atomic absorption analysis. They also are atso used for other analysis, such as for uranium by laser fluorimetry, to help determine potential interference effects. The analytical spike is essentially the same as a post-digestion spike. However, because the analytical spike is applied to all samples and the post-digestion spike is applied exclusively to the matrix spike sample, different nomenclature is applied.

Recovery of the analytical spike is determined by subtracting the spike sample concentration from the accompanying sample concentration and dividing the difference by the amount spiked. Recoveries outside of the 75-percent to 125 - percent parameters warrant investigation and corrective action. Corrective action may consist of dilution followed by reanalysis, the use of standard additions or, in extreme cases, repreparation of the sample followed by reanalysis. 


\subsubsection{Post-Digestion Spike}

| For inorganic analytes of interest, a post-digestion spike (PDS) is a spike added to the sample after preliminary preparation, usually just before analysis. The PDS is used to indicate matrix-related interferences on the analytical system that may still be present in the sample following digestion. The PDS is normally used when a matrix spike failure occurs. This | technique is typically used for ICP analysis, but is appropriate to other analyses as well.

PDS performance can be used to identify problems caused by sample matrix effects during the sample preparation and/or measurement. In case of unusual matrix effects only during the preparation step, the MS will fail but the PDS will pass. If both the MS and PDS fail, the failure is caused by matrix effects in both the preparation and measurements steps.

Acceptable recovery is generally 75 percent to 125 percent for the PDS. If the PDS meets the acceptance criteria, indicating that MS failure occurred during the sample preparation step, the results of all client samples in the batch should be flagged in the laboratory report and the sample preparation problem pointed out in the case narrative. If the PDS fails to meet the acceptance criteria, this indicates a failure in both the sample preparation and measurement steps caused by matrix problems. All results of client samples in the batch should be flagged and the reasons discussed in the case narrative.

Reanalysis of the samples using different preparation and/or measurement procedures should be considered by the laboratory if alternative procedures are available that, in the judgement of qualified chemists, offer a reasonable solution to the problemfs). Before reanalysis, client concurrence must be obtained because the procedural changes may violate mandated existing regulatory or project requirements.

\subsubsection{Serial Dilution}

When new or unusual matrices are encountered, serial dilution is used for ICP analysis-as an indicator of potential matrix-related interferences associated with analysis. Serial dilution is simply a fivefold dilution of a sample (after all preparation steps are complete) followed by analysis. Serial dilution is ontyperformed only when a sufficient number of target analyte concentrations in the client sample exceed 50 times the IDL. When sample analyte concentration is less than 50 times the IDL, an analytical spike should be performed.

The serial dilution is designed to indicate potential problems, such as high solids effects, that can affect sample uptake, resulting in analyte measurement differences. In these cases, results would begin to vary beyond the 10 percent criterion because of sample aspiration and the subsequent effect on analyte species detected. The serial dilution does not replace a sample dilution necessary to maintain a sample in optimum instrument performance range.

A percent difference result of 10 percent or less; between the original and the diluted sample indicates no significant matrix effects during the measurement process. The client sample results 
I in the batch that does not meet the serial dilution acceptance criterion should be noted and possible matrix effects discussed in the case narrative.

\subsubsection{Method of Standard Additions}

| The method of standard additions consists of a blank and at least three standards to which aliquots of the sample are added. The standards used should be approximately 50 percent, 100 percent, and 150 percent of the expected sample concentration. The method of standard additions can be used in lieu of instrument calibration because each sample essentially has its own calibration. However, the QC presented in Section 12.4 is still required (including "auto-zeroing" on the calibration blank). The method of standard additions is meant to compensate for a sample matrix effect that enhances or depresses analyte signals.

\subsection{ORGANIC PREPARATION TECHNIQUES}

Preparation techniques are used to prepare a sample for analysis, examples of preparative techniques include: digestion, dissolution, extraction, phase partitioning, and/or leaching of a I sample material. Separation and/or isolation techniques also are considered preparative if performed before analysis to facilitate reliable analyte measurement. Simple dilution is not a sample preparation technique. Selection of preparation techniques should be based on the I client's data quality requirements.

HASQARD considers the use of either CLP protocols or associated SW-846 (EPA 1992) protocols to be acceptable because applicable SW- 846 methods are based on early CLP statements of work (that is, based on the CLP statement of work, August 1994 Revision)

\subsubsection{Preparation Batch}

A preparation batch is a group of samples; of similar matrix type- prepared at the same time. A batch shall not exceed 20 samples, excluding the QC samples (for example, blank, matrix spike, duplicate). More than 20 samples can be prepared at the same time, if the required number of $Q C$ samples are performed for each batch. If clients do not specify project-specific QC (which requires the laboratory to select client samples for duplicates and matrix spikes), the laboratory may combine up to 20 samples of a similar matrix type for preparation with only one matrix spike duplicate for duplicate) and matrix spike required. However, the preparation blank and LCS or blank spike requirements are-always are applied to each batch of samples.

A sample or series of samples that does not require preparation or separation before analysis would not fall under the requirements of this section. Additionally, limited sample quantity may limit the laboratory's ability to meet the duplicate and matrix spike requirements. In such cases, alternative approaches should be considered to demonstrate sample precision and accuracy. Unique client requirements shall be documented and agreed upon by the laboratory and the client before work begins. 


\subsubsection{Preparation Blank (Method Blank)}

The sample preparation blank (method blank) is used to monitor contamination resulting from the sample preparation process. The preparation blank is generally distilled or deionized water (reagent)that is subjected to the same processing as the samples, including all reagent additions. I The laboratory is also is free to use a matrix more closely related to the actual samples processed, provided the matrix is free of contamination from analytes of interest (for example, clean soil or sand for solid matrices). Interferences and ancillary contamination produced by the blank material would be expected to be similar to that of the sample. The preparation blank volume or weight shall be approximately equal to the sample volume or weight being processed.

Preparation or method blank acceptability shall be demonstrated as follows: 1) the blank shall be less than the EQL of the associated samples, or, if the blank is equal to or above the EQL, 2) it shall not exceed 5 percent of the measured concentration present in the associated samples, or, when a decision level is specified, 3) the blank shall not exceed 5 percent of the decision level unless item 2)applies. Note: The laboratory must be capable of achieving an EQL less than or equal to 5 percent of the decision level or must negotiate an acceptable alternative.

As a special requirement, the concentration of the following compounds shall be less than five times the EQL:

$\begin{array}{ll}\text { - } & \text { Methylene chloride } \\ \text { - } & \text { Acetone } \\ \text { - } & \text { Phutanone } \\ & \text { Phthalate esters. }\end{array}$

All samples in the preparation batch will be reprepared and reanalyzed if the preparation blank (method blank) fails to meet one of the acceptance criteria for a compound of interest.

The results of the preparation blank analysis shall be reported to the client as part of routine reports. Preparation blank results are not subtracted from sample results unless client data quality requirements specify otherwise, in which case the narrative accompanying the results should state that blank subtraction was used.

Preparation blanks or method blanks shall be prepared with each batch of samples.

\subsubsection{Laboratory Control Sample or Blank Spike}

The LCS or blank spike shall be used to monitor the effectiveness of the sample preparation process. An LCS is a material similar in nature to the sample being processed, containing known concentrations of the analyte of interest (for example, standard reference material). The LCS is subject to the same processing (preparation, separation, analysis, and so forth) as the client samples. A blank spike is normally used when an appropriate LCS is unavailable. The blank spike is distilled or deionized (reagent) water spiked with the analytetsj of interest. 
Results from the analysis of the LCS or blank spike are compared to the analyte values known to be present in the sample. LCS acceptability is demonstrated by target analytes being within 80 percent to 120 percent or within SPC limits when the \pm 20 percent criteria cannot be reliably achieved. Control limits are established statistically, as determined by multiple analyses over time. New methods will use administrative (vendor, regulatory, or past experience) limits until enough data can be gathered to set limits statistically.

When one of the acceptance criteria for the LCS has not been met for an analyte of interest, all samples in the preparation batch shall be reprepared and reanalyzed for that analyte. Client criteria will not override LCS control limits unless agreed tpon with the client before the analysis in accordance with HASQARD, Volume 1, pages 1-7.

\subsubsection{Matrix Spike}

A matrix spike is a client sample that has been spiked with the analyte of interest and processed in the same manner as the sample. The matrix spike is used to monitor method performance in a specific sample matrix. Matrix spike results are a measure of the bias (difference from true value) in the measurement of the target analytes introduced by the client sample matrix.

When the sample concentration is unknown, spiking is typically performed at a level that is one of the following:

\footnotetext{
- Equivalent to the regulatory threshold

- Specified by method

- One to five times the EQL.
}

Otherwise, the spiking should be performed at a level equivalent to one to two times that of the sample, the general spiking rule is "the spike concentration must be greater than or equal to 25 percent of the sample concentration." However, general EPA spiking guidelines state that spike recovery limits do not apply when sample concentration exceeds the spike concentration by a factor of four (EPA 4X Rule) or more. (For a sample concentration of 500 ppm; a valid spike concentration, added to the sample, would need to be between the minimum of $125 \mathrm{ppm}$ and a maximum of $2,000 \mathrm{ppm}$.) When the concentration of the analyte in the original sample is too large (chemist evaluation), no matrix spike is required.

For organic analysis, matrix spike control is demonstrated when target compounds are within established control limits. The control limits identified in Methods for Evaluating Solid Waste, Physical/Chemical Methods (SW-846) (EPA 1992) or the current CLP statement of work are acceptable. The selection of limits is based on which method (SW-846 or CLP) the laboratory uses. Control limits are typically established by recommended regulatory limits, laboratory performance over time, or the client by the data quality requirements for a particular project or program. For compounds of interest or matrices not covered by SW-846 or CLP, the laboratory shall establish spike compounds, spike levels, and acceptable recovery criteriaas described in Chapter 5 . 
If the matrix spike recovery fails to meet the criteria, the batch shall be investigated for sources of error. Repreparation and/or reanalysis should be conducted as necessary based on the DQO and DQR. The goal is to ensure that limitations on the data caused by the sample matrix and represented by matrix spike performance, are adequately portrayed and discussed in the report to the client.

In those instances where the sample concentration significantly exceeds the amount of spike added in the prepared samples, the data must be further evaluated to determine if the recovery of the spiked sample is meaningful.

A matrix spike shall be prepared with each batch of 20 samples and the results reported to the client along with the calculated recovery (see Section 12.2.1). No adjustment of the client sample results is made in the laboratory report.

\subsubsection{Laboratory Sample Duplicate or Matrix Spike Duplicate}

Laboratory sample duplicates are two aliquots of the same sample (intralaboratory split) that are taken through the entire sample preparation and analytical process. Laboratory duplicates are used to assess the precision of the preparation and analysis process in a client-specific matrix. Agreement between duplicates indicates the reproducibility of the combined preparation, separation, and measurement process (precision).

Matrix spike duplicates are two spiked aliquots of the same sample that are taken through the entire sample preparation and analytical process. Where the sample is not expected to contain reasonable concentrations of the analytes of interest, duplicate sample results will not provide a reliable estimate of precision. In these cases, matrix spike duplicates are used to demonstrate analytical precision in the client sample.

Agreement between duplicates indicates reproducibility of the combined preparation, separation, and measurement process (precision).

Disagreement can occur because of analyte concentration differences within the sample matrix (nonhomogeneity) that are not amenable to analyst control during analysis. Disagreement may arise if the method has poor applicability to the analyte or matrix tested. Typically, organic analyses include a matrix spike and matrix spike duplicate because a low probability exists that the majority of those analytes of interest will be detected in the sample.

Organic matrix spike duplicate RPD criteria vary widely according to analyte and method. They are very method and matrix dependent. Acceptance criteria are established by in one of the following ways: 1) They are specified by regulatory requirement. 2) They are specified by the client via the DQO process for a particular project. 3) They are determined by laboratory performance over time for samples with similar matrices and concentration ranges.

One set of matrix spike or matrix spike duplicates is required for each batch of samples. Precision is calculated using the RPD. 
The results of duplicate (or matrix spike duplicate) analyses are reported to the client along with the calculated RPD for each analyte.

The run ends based on analytical clock expiration for organic analyses by GC/MS. For the gas chromatography and carbon analysis, the run ends based upon continuing calibration performance.

\subsubsection{Initial Calibration Verification}

The initial calibration verification is a standard used to confirm the acceptability of the calibration and the standards used to prepare the calibration. Acceptable performance of the initial calibration verification demonstrates that both the standards used and the instrument are functioning properly. The initial calibration verification is prepared from a source other than that used to prepare the calibration standards (see Chapter 7).

Analytical measurement systems for which calibration applies over an extended period of time (that is, months for some GC/MS methods) normally use the initial calibration verification only at the time of calibration. Subsequent, routine performance checks are made using the equivalent of a continuing calibration verification (see Section 11.6.3).

Analytical measurement systems that are calibrated frequently and for which calibration standards are routinely prepared normally follow calibration with an initial calibration verification.

Failure of the initial calibration verification indicates instrument and/or standard problems that shall be evaluated and corrected before any samples are processed for the analytes of interest.

\subsubsection{Continuing Calibration Verification}

The CCV analytical standard is used to monitor instrument stability over time. Acceptable performance demonstrates the continued accuracy of the calibration. The CCV may be prepared from any reliable source. The initial calibration verification $\mathrm{QC}$ sample may be used as the $\mathrm{CCV}$ sample.

The calibration of organic analytical GC/MS methods is limited by analytical run time. Each run is defined by a 12-hour clock that cannot be exceeded. Each run starts with a continuing calibration verification (after tuning). The analyst shall rely on internal standard and surrogate performance to ensure that the sample run ended in control. Corrective action shall be taken. These include, such actions as reanalysis of all samples demonstrating unacceptable internal standard performance

Analytical runs for organic analysis such as GC typically can extend anywhere from several hours to several days. Continuing calibration checks are required throughout the run duration(for example, approximately every 10 samples for SW-846, every 12 hours for CLP). Total organic carbon; and total carbon measurements also should include continuing calibration verification checks every 15 samples. 


\subsubsection{Continuing Calibration Blank}

Continuing calibration blanks (that is, instrument blanks) monitor effects such as contamination and instrument response drift. The continuing calibration blank (CCB) is a reagent blank prepared similarly to the standards, but not subjected to preliminary sample preparation except for techniques in which the preparation is an integral part of the analysis (that is, purge and trap procedures). In these cases, the preparation blank or method blank can be considered the equivalent of the initial calibration blank or $\mathrm{CCB}$. The generally accepted criterion for reagent blanks is that they are below the EQL for each analyte tested. When an analyte exceeds this criterion, the analysis shall be investigated for potential impact. Samples with target analyte concentrations exceeding the blank contamination level by a factor of 20 or more can be reported unless client requirements dictate otherwise. In all other cases, all samples analyzed since the last acceptable initial calibration blank or CCB shall be reanalyzed for the specific analyte failure.

Several organic compounds are more readily introduced into blanks; see Section 11.7.2 for these specific exceptions. In the case of organic analysis by $\mathrm{GC} / \mathrm{MS}$, the blank is typically the equivalent of the method blank. The method blank is run after the continuing calibration verification standard. Periodic calibration blanks are not performed. In the case of organic analysis by gas chromatography and carbon analysis, periodic blanks are recommended following each CCV.

The CCB problems should be corrected, then the samples analyzed after the last successful CCB should be reanalyzed.

\subsubsection{Internal Standards}

An internal standard is an analyte that is similar to the analyte of interest in chemical composition and analytical response but is not normally expected to be found in the sample. Internal standards are added to every standard, blank, matrix spike, matrix spike duplicate, and sample before analysis. Internal standards are used as the basis for quantitation of the analytes of interest.

Selecting appropriate internal standards shall be method and compound-list specific because all results are normalized based on internal standard performance. Laboratory procedures shall specify acceptance criteria.

Internal standards are used in organic GC/MS analysis although they also may be appropriate to other types of analysis. Generally, area counts of less than 50 percent or more than 150 percent of original area counts in the continuing calibration standard are considered unacceptable.

\subsubsection{Low-Level Standard}

The low-level standard is used to monitor instrument performance in the region near the EQL. When the low-level standard concentration is included in the instrument calibration, a separate 
low-level standard is not required. Low-level standards are not required for total organic carbon, total inorganic carbon, and total carbon.

\subsection{PROCEDURES TO ASSESS DATA QUALITY}

I This section provides formulas that typically are used to compute quality assurance parameters used to assess data quality. These quality control parameters should be monitored, evaluated, and/or trended on short-term and long-term bases. For example, system contamination control (blank or background activity), precision, accuracy, spike recovery, and tracer or carrier yield recovery could be evaluated based on method, matrix, and activity or concentration level. . Such activities provide a basis for continuous quality improvement and insight on overall laboratory performance.

\subsection{PRECISION}

| Precision was define inChapter 4. Precision for the sample is estimated by analyzing duplicate and/or replicate, or matrix-spike duplicate samples. Samples used to calculate precision should contain the concentrations of analytes above the $\mathrm{MDL}$ or $\mathrm{EQL}$. The precision of test results is expressed as the RSD or the RPD. The precision of a method in a given matrix is expressed as the RSD or the RPD among matrix spike duplicates.

In addition to precision determined by the sample duplicate or matrix spike duplicate, precision for the standards (for example, laboratory control sample and continuing calibration verification standard) can be calculated and used to monitor quality control of the analytical measurement system over time. Precision of the sample ean also can be monitored for long-term quality control, but should be based on method, matrix, and activityf or concentration in the sample.

\subsubsection{Relative Standard Deviation}

The RSD is used when at least three replicate measurements are performed on a.given technique. The RSD is computed using the following equation:

$$
\mathrm{RSD}=\frac{\mathrm{s}}{\overline{\mathrm{x}}} * 100
$$

where
$\mathrm{s}=\quad$ Standard deviation with $\mathrm{n}-1$ degrees of freedom, $(\mathrm{n}=$ total number of observed values)
$\overline{\mathrm{x}}=$ Mean of observed values. 


$$
\text { HNF-SD-WM-QAPP-034 Rev. } 3
$$

Precision was define inChapter 4. Precision for the sample is estimated by analyzing duplicate and/or replicate, or matrix-spike duplicate samples. Samples used to calculate precision should contain the concentrations of analytes above the MDL or EQL. The precision of test results is expressed as the RSD or the RPD. The precision of a method in a given matrix is expressed as the RSD or the RPD among matrix spike duplicates.

In addition to precision determined by the sample duplicate or matrix spike duplicate, precision for the standards (for example, laboratory control sample and continuing calibration verification standard) can be calculated and used to monitor quality control of the analytical measurement system over time. Precision of the sample eanalso can be monitored for long-term quality control, but should be based on method, matrix, and activityf or concentration in the sample.

\subsubsection{Relative Standard Deviation}

The RSD is used when at least three replicate measurements are performed on a given technique. The RSD is computed using the following equation:

$$
\mathrm{RSD}=\frac{\mathrm{s}}{\overline{\mathrm{x}}} * 100
$$

where
$\mathrm{s} \quad=$
Standard deviation with $n-1$ degrees of freedom, $(n=$ total number of observed values)
$\overrightarrow{\mathrm{x}}=$ Mean of observed values. 


\subsubsection{Relative Percent Difference}

The RPD is used when two measurements exist. The RPD is-generally is used to express the precision of matrix duplicate or matrix spike duplicate samples. The RPD is computed using the following equation:

$$
\mathrm{RPD}=\frac{\left|\mathrm{x}_{1}-\mathrm{x}_{2}\right|}{\overline{\mathrm{x}}} * 100
$$

where

$$
\begin{array}{lll}
\mathrm{x}_{1} \text { and } \mathrm{x}_{2} & = & \text { Observed values } \\
\overline{\mathrm{x}} & = & \text { Mean of observed values. }
\end{array}
$$

\subsection{ACCURACY}

\subsubsection{Method Accuracy Based on Sample Spike}

Accuracy was defined in Chapter 4. Accuracy for the sample is expressed as the percent recovery (\%R) of a matrix spike (or matrix spike duplicate) sample. The percent recovery is calculated based on the following equation:

$$
\% \mathrm{R}=\frac{(\mathrm{SSR}-\mathrm{SR})}{\mathrm{SA}} * 100
$$

where

$$
\begin{array}{lll}
\text { SSR } & = & \text { Spiked sample result } \\
\text { SR } & = & \text { Sample result } \\
\text { SA }= & \text { Spike added. }
\end{array}
$$

\subsubsection{Method Accuracy Based on Standard}

The accuracy of an analytical method is expressed as the percent recovery of a standard (\%R). The percent recovery of a standard is calculated according to the following equation:

$$
\% \mathrm{R}=\frac{\mathrm{A}_{\mathrm{m}}}{\mathrm{A}_{\mathrm{k}}} * 100
$$


where
$A_{\mathrm{m}} \quad=\quad$ Measured value of the standard analyte
$A_{k}=$ Known value of the standard analyte.

\subsection{MEASURES OF AGREEMENT}

\subsubsection{Percent Difference}

The percent difference $(\% \mathrm{D})$ is often used to compare one reference point to another. The percent difference is calculated using the following equation:

$$
\% D=\frac{|I-C|}{I} * 100
$$

where

$$
\begin{array}{lll}
\mathrm{I}= & \text { Observed value used as the reference point } \\
\mathrm{C}= & \text { Compared value. }
\end{array}
$$

\subsubsection{Bias}

1 Bias (B) often is used to measure the deviation of a measured value from a known value or accepted reference value. Bias can be assessed by comparing a measured value to an accepted reference value in a sample of known concentration or by determining the recovery of a known amount of contaminant spiked into a sample. Thus, the bias caused by the matrix effects based on a matrix spike is calculated using the following equation:

$$
B=\left(X_{s}-X_{u}\right)-K
$$

where

$$
\begin{array}{lll}
\mathrm{X}_{\mathrm{s}}= & \text { Measured value (for example, spiked sample) } \\
\mathrm{X}_{\mathrm{u}}= & \begin{array}{l}
\text { Miscellaneous contribution (for example, sample contribution). If no } \\
\text { miscellaneous contributions exist, } \mathrm{X}_{\mathrm{u}} \text { would be zero. }
\end{array} \\
\mathrm{K} & =\quad \text { Known value (for example, true spiked value) }
\end{array}
$$




\subsubsection{Mean Difference}

Mean difference may be used to compare two duplicate results and is generally used for radiochemical analysis. The mean difference takes into account the uncertainty associated with each measurement. The mean difference is compared based on a two-sided $z$-test for a known population (Steel and Torrie 1960) and is calculated using the following equation:

$$
M D=\frac{\left|R_{1}-R_{2}\right|}{\sqrt{\left.\left(a_{1}^{2}\right)+a_{2}^{2}\right)}}
$$

where

$\begin{array}{lll}\mathrm{R}_{1}= & \text { First sample result } \\ \mathrm{R}_{2}= & \text { Second sample result } \\ \mathrm{a}_{1}= & \text { One sigma uncertainty of first result } \\ \mathrm{a}_{2}= & \text { One sigma uncertainty of second result. }\end{array}$

If the $\mathrm{MD}$ is greater than or equal to 1.96 , a 95 percent confidence exists that the two results are not equal.

\subsection{DETECTION LIMIT CONSIDERATIONS}

\subsubsection{Inorganic And Organic Methods}

13.4.1.1 Method Detection Limit. The MDL is defined as "the minimum concentration of a substance that can be measured and reported with 99 percent confidence that the value is greater than zero" (SW-846, consistent with the requirements specified in 40 CFR 40, Appendix B, to 40 CFR 136) and is briefly described in the following text.

The concentration of the MDL for the analyte of concern can be estimated by using one of the following:

- Instrument signal-to-noise ratio within the range of 2.5 to 5

- Region of the standard curve where there-is a significant change in sensitivity occurs (that is, a break in the slope of the standard curve).

When determining the MDL, a minimum of three analyses are required in a matrix spiking with the analyte of interest at a concentration three to five times the estimated $\mathrm{MDL}$. Whenever possible, the matrix should be similar to the sample matrix. When not possible, the matrix will be 
water. All sample processing steps of the analytical method shall be included in the final determination of the MDL.

Variance $\left(\mathrm{S}^{2}\right)$ is determined from the replicate measurements, as shown:

$$
s^{2}=\frac{1}{(n-1)}\left[\sum_{i=1}^{n}\left(X_{i}-\bar{X}\right)^{2}\right]
$$

where

$$
\begin{array}{lll}
X_{i} & = & \text { With measurement of the variable } X \\
X & = & \text { Mean of observed variable } X .
\end{array}
$$

The MDL should be determined by the following equation:

$$
\mathrm{MDL}=\mathrm{t}(\mathrm{n}-1, \alpha=0.99) *(\mathrm{~s})
$$

where

$$
\begin{array}{ll}
t_{(n-1, n=0.99)}= & \begin{array}{l}
\text { One-sided t-statistical value appropriate for the number of samples used } \\
\text { to determine standard deviation }
\end{array} \\
\mathrm{s} & =\quad \text { Standard deviation obtained from the MOL replicate measurements. }
\end{array}
$$

13.4.1.2 Instrument Detection Limit. The IDL is the smallest signal above background noise that an instrument can detect reliably. The $\mathrm{DL}$, is determined by spiking reagent water with each analyte of concern. The following considerations apply to the selection of the IDL standard.

- Concentration of the IDL standard should be at least equal to or in the same concentration range as the estimated IDL.

- Concentration of the IDL standard should be in the region of the standard curve where -a significant change in sensitivity occurs.

A minimum of seven aliquots of the IDL standard are required to determine the IDL. The IDL standards are run through the analytical process only. The IDL is calculated using the same equation as the MDL.

Ideally, each analytical method should have data supporting an MDL for the specific matrix of each sample. If this is not practical, MDL on generic matrices (for example, deionized water, ground water, soil) can be used to estimate the sample-specific MDL. If the MDL cannot be determined, at a minimum, IDL information will be available and can be used.

13.4.1.3 Estimated Quantitation Limit. The EQL has been defined by RCRA as the lowest level that can be reliably achieved within specified limits of precision and accuracy under routine 
laboratory operating conditions. EQL is often referred to as practical quantitation limit (PQL) by clients in their work authorization documents. The analyte concentration at the estimated quantitation limit is determined using the following guidance: 1) 5 to $10 \mathrm{XMDL}$ or $\mathrm{IDL}$, or 2) the lowest non-zero standard in the calibration curve. In some cases; sample dilutions affect the quantitation limit. The quantitation limit for this sample is calculated as dilution factor times the EQL as defined by RCRA.

The quantitation limit can be used to qualify the sample results or compare the data quality requirements from the end user of the data during the DQO process. When results are less than the quantitation limit or detection limit, sample results should be reported with the appropriate qualifier. Results that are less than the IDL or MDL are reported as the numeric limit proceeded by a less than If the detection limit is reported elsewhere, the result may be reported as undetectable. Results falling between the IDL (or MDL) and the EQL can be reported with the appropriate qualifier or discussed in the case narrative.

13.4.1.4 Minimum Detectable Activity. The minimum detectable activity (MDA) has been defined as a level of activity that is practicably achievable by a measurement system. The sample MDA generally is applied as the mean (expected) activity of samples having a 5 percent probability of escaping detection arid a 5 percent probability of false detection. The MDA is calculated based on Currie's formula (Currie 1968) and is simplified to the following two equations when the counting time in the sample is the same as in the background. It must be recognized that the background may, and normally is, affected by the components of the sample. Therefore, the MDA value is an (a posteriori) estimate for comparative purposes.

$$
\begin{gathered}
\mathrm{MDA}=\left[\left(\frac{2.71}{\mathrm{~T}}\right)+(2 * \mathrm{DLR})\right] / \mathrm{K} \\
\text { or } \\
\mathrm{MDA}=\left[\left(\frac{2.71}{\mathrm{~T}}\right)+\left(4.65 * \mathrm{~S}_{\mathrm{b}}\right)\right] / \mathrm{K}
\end{gathered}
$$

where

$$
\begin{array}{ll}
\mathrm{T}= & \text { Sample count time } \\
\mathrm{K}= & \text { Detector calibration factor (for example, } \min ^{-1} / \mu \mathrm{Ci} \text { or s} \mathrm{s}^{-1} / \mu \mathrm{Ci} \text { ) } \\
\mathrm{S}_{\mathrm{b}}= & \text { Standard deviation of background count rate for the counting time (T). }
\end{array}
$$

When $T_{b}$ is not equal to $T_{t}, M D A$ is calculated as followsshow. 


$$
\mathrm{MDA}=\frac{2.71+3.3}{\cdot\left(\mathrm{R}_{\mathrm{b}} * \mathrm{~T}_{\mathrm{b}}\right) *\left(1+\frac{\mathrm{T}_{\mathrm{b}}}{\mathrm{T}_{\mathrm{t}}}\right)}
$$

where

$\begin{array}{lll}\mathrm{R}_{\mathrm{b}} & =\text { Background count rate } \\ \mathrm{T}_{\mathrm{b}} & =\text { Background count time } \\ \mathrm{T}_{\mathrm{t}} & =\text { Sample count time } \\ \mathrm{e} & =\text { Counting efficiency } \\ \mathrm{b} & =\text { Abundance } \\ \mathrm{L}_{\mathrm{T}} & =\text { Elapsed live time (background counting time }=\mathrm{T}_{\mathrm{b}} \text { ) } \\ \mathrm{k} & =\text { Conversion factor (for example, } 37,000 \text { disintegrations } / \text { second } / \mu \mathrm{Ci} \text { ). }\end{array}$

The MDC is defined as the mean concentration of samples having a 5 percent probability of escaping detection and a 5 percent probability of false detection.

$$
\mathrm{MDC}=\frac{\mathrm{MDA}}{\mathrm{q} * \mathrm{Y} * \text { decay }}
$$

where

$$
\begin{array}{lll}
\mathrm{q} & = & \text { Sample quantity (for example, } \mathrm{g} \text { or } \mathrm{mL} \text { ) } \\
\mathrm{Y} & = & \text { Chemical yield } \\
\text { decay } & = & \text { Decay factor (correction for radioactive decay to reference date) }
\end{array}
$$

Software provided by vendors may have variations of thetothis formula. A vendor-provided software or data-reduction package is adequate for data calculation.

13.4.1.5 A Priori and $a$ Posteriori Concepts and Information. Decision-level count rate, $M D A$, and $M D C$ are considered a priori (before the measurement). The estimation of these quantities requires specification of nominal values of a number of parameters (for example, background count rate, count time, estimated interferences, chemical recoveries, decay times). Assessment of a priori detection limit parameters for future measurements may require the knowledge of a posteriori information concerning the nominal characteristics of the sample batch gained from preliminary measurements.

The question of whether the sample contains net activity is best answered by comparing the measurement result to the detection level or considering the confidence interval for the measurement result, not by comparing the result to the estimated MDA or MDC. 


\subsubsection{Limit of Detection}

In some cases, the limit of detection is used. The limit of detection represents a criterion for detection decision, that is, deciding whether to classify a result as detected or not detected when the observed signal is close to that obtained for blank measurements (that is, similar to background noise).

\subsection{UNCERTAINTY}

Uncertainty is expressed as the range of values within which the true value is estimated to lie. The uncertainty estimate consists of two components, systematic and random variability. Each contributing source of uncertainty is expected to be distributed over its range. Each systematic component can be estimated in terms of the measurement result for the contributing source of uncertainty.

The analytical systematic component can be estimated using standard or spike recovery. The random analytical component can be estimated from replicate measurements of a sample.

The total uncertainty is calculated as the square root of the sum of the squares of random and systematic variabilities as shown in the following equation. The component of uncertainty has to be expressed in the same unit designation (for example, concentration percentage).

$$
\text { Total uncertainty }=\sqrt{\left(s_{x}^{2}\right)+\sum_{j=1}^{q} \delta_{j}^{2}}
$$

where

$$
\begin{array}{lll}
\mathrm{s}_{\mathrm{x}} & =\text { Standard error } \\
\mathrm{q} & = & \text { Number of systematic uncertainty components } \\
\delta & = & \text { Systematic uncertainties. }
\end{array}
$$

Uncertainty is used in the radiochemical analyses to express method and counting error. The total random uncertainty is obtained by propagating the individual variance $\left(\mathrm{s}_{\mathrm{i}}^{2}\right)$ and is expressed as the standard error based on multiple determinations of $x$. However, the typical radiochemical methods used are not sufficient to separate systematic and random uncertainties suehto the extent that biases can be corrected. Uncertainty will be measured, or uncertainty will be estimated if it cannot be measured. 


$$
\text { HNF-SD-WM-QAPP-034 Rev. } 3
$$

\subsection{CONTROL CHARTS}

All control charts statistics to determine if the random error of a result exceeds the control limits established for a particular method. Control charts shall be used to determine whether or not a particular analytical procedure is "in control" (the results being plotted lie within expected limits of precision)or "out of control" (results lie outside expected precision limits), or shows a trend indicating that something other than random error is affecting results. The SAS laboratory will use control charts based on standard solutions (LCS recoveries), blanks, or customer requirements. For best results, at least 20 determinations should be made before calculating the mean and standard deviation. These determinations need not be made on the same day. Control charts shall be used by the chemist responsible for the instrument measurement system to document method performance. Requirements for control charts are documented in LQ-150-001. | Control charts are a useful tool for assessing trends in system performance. Monitoring these trends by the technical staff will help them do the following:

- Ensure their analytical system is in control

- Recognize problems and address them before the system goes out of control

- Document conditions or corrective actions that fix particular performance problems for future reference)

- Demonstrate continuous quality improvement as they refine the system and practices.

At a minimum, control charts are maintained for a parameter or parameters that monitor both the preparation process (if any) and the analytical measurement. Normally this requirement can be fulfilled by charting the LCS recovery percent.

Control limits will be set either at 3 sigma or based on environmental regulatory requirements. Any data point that falls outside the upper or lower control limit requires the chemist investigation to investigate and take corrective action. Trends or patterns should be noted and investigated. Trends or patterns also should also-be relayed to QC personnel for further evaluation in consultation with the responsible chemist.

The cognizant chemist may choose (and is encouraged) to chart other parameters that assist in monitoring instrument or preparation procedures. Such examples include internal check standards, instrument control standards, signal intensities, lamp energies, or background levels.

The control charts will be maintained in such a way that they are readily available to the laboratory technologist, cognizant chemist, QA/QC representatives, or management. Control charts shall be kept current for immediate use and may take the form of at-the-bench manually updated charts, on-line electronic displays or hard-copy printouts generated periodically as close to real time as practical.

The technical staff will be trained on the maintenance and use of control charts and be able to use them to demonstrate that their measurement system is in control. 


$$
\text { HNF-SD-WM-QAPP-034 ReV. } 3
$$

\subsection{MISCELLANEOUS CALCULATIONS}

\subsubsection{Response Factor}

The response factor (RF)is calculated to compare a compound to an internal standard based on the ratio of the area response of the characteristic ions against the concentration. The internal standard selected for calculating the RF for a compound should be the internal standard with a retention time closest to the compound being measured. The RF is computed using the following equation:

$$
R F=\frac{\left(A_{x}\right)\left(C_{i s}\right)}{\left(A_{i s}\right)\left(C_{x}\right)}
$$

where
$\mathrm{A}_{\mathrm{x}} \quad=\quad$ Area of the characteristic ion for the compound being measured
$\mathrm{C}_{\mathrm{is}}=$ Concentration of the specific internal standard
$\mathrm{A}_{\mathrm{is}}=$ Area of the characteristic ion for the specific internal standard
$\mathrm{C}_{\mathrm{x}}=$ Concentration of the compound being measured.

\subsubsection{Percent Difference of the Response Factor}

The percent difference $(\% \mathrm{D})$ is used to compare the $\mathrm{RF}$ of the continuing calibration verification compounds against the RF of the initial calibration compound. The percent difference is used to compare daily GC/MS performance against a basis established during initial calibration.

The percent difference to compare the RF is computed in the following equation:

$$
\% D=\frac{\left(R F_{I}-R F_{c}\right)}{R F_{I}} * 100
$$

where

$$
\begin{aligned}
& R F_{1}=\text { Average } R F \text { from initial calibration } \\
& R F_{c}=R F \text { from continuous verification check standard. }
\end{aligned}
$$


HNF-SD-WM-QAPP-034 Rev. 3

This page intentionally left blank. 


\subsection{ASSESSMENTS}

A QA program can be effective only if systems are in place to continuously monitor or assess the laboratory's ability to conform to program requirements. A QA program is effective only if meaningful assessment systems are in place to continuously monitor, assess, and respond to issues associated with program or organizational performance.

Assessment tools can include management system assessments; and technical system assessments (surveillances, performance evaluations, data quality assessments [reviews], peer and technical reviews, readiness reviews, and/or external audits and assessments).

At a minimum, the laboratory assessment program shall address the following:

- Management system assessments

- Technical system assessments

- Performance evaluation (PE) assessments

- Data quality assessments (reviews)

- External assessments.

To achieve this goal, personnel who perform independent assessments shall be technically qualified and have sufficient authority and freedom from the line organization to carry out their responsibility. The independent assessment process shall incorporate a performance-based approach with emphasis on the results of the work process and compliance to requirements.

The QA program consists of management assessments, technical systems audits, performance audits, data quality audits, and external audits. The procedures for performing laboratory assessments are described in Waste Management Hanford Procedure, WMH-200, No. 1.2. Individual performance evaluations are driven by $\mathrm{PE}$ program protocol.

Results and corrective actions from the external audits are tracked by the DTS. The technical support staff track and trend corrective actions to closure and distribute documentation to the responsible manager and staff.

\subsection{MANAGEMENT ASSESSMENTS}

Management system assessments are directed by those immediately responsible for overseeing and/or performing the work. Managers shall assess their management practices. This QA program provides a solid basis for this assessment. (Refer to HNF-PRO-246 and WMH 200, No. 1.2.) The purpose of this assessment is to evaluate the following:

- Effectiveness of management control systems established to achieve and ensure quality. 
- Adequacy of resources and personnel available to achieve quality objectives to which the quality systems apply

- Effectiveness of training and assessment

- Applicability of data quality requirements.

SAS management assessments are performed using WMH-200, Section 1.2. These assessments are scheduled over a 3-year cycle and cover eight topics:

- Quality assurance

- Training and qualifications

- Conduct of operations and maintenance

- Maintenance

- Radiation protection

- Environmental management

- Occupational safety

- Engineering services.

\subsection{TECHNICAL SYSTEMS ASSESSMENTS}

Technical system assessments (reviews, surveillances, evaluations) are directed by the laboratory, program, or QA function. These assessments measures the performance or effectiveness of the technical system and its elements with respect to documented specifications and objectives. Technical system assessments consist of a review of laboratory operations, specific procedures, and related documentation. For example, areas of interest might include the following:

- Maintenance and Testing Equipment (M\&TE) calibration or control procedures

- Document control procedures

- Technical procedure compliance

- Adherence to data quality requirements

- Identification, control, storage, and preservation of samples or standards

- Communication of client expectations.

\subsection{PERFORMANCE AUDITS}

Performance audits (typically referred to as performance evaluations) are generally considered blind or double-blind tests introduced into a process to-independently evaluate the quality of the process. Performance evaluations are compared with results from other participating laboratories.

Laboratory QA function should coordinate PEs whenever practical to avoid any conflict of interest. Quality Systems describes the following programs in applicable desk instructions to assist in sample receipt, documentation, reporting, and performance. 
A strong PE program will typically consist of internal and external performance measures. However, a program based upon external blinds is considered the minimum acceptable.

The Hanford Performance Evaluation Program was established (Markel 1996) to provide a central point of coordination and control for external and internal programs, and to evaluate and qualify laboratories performing analyses of samples from the Hanford Site. A Hanford Site performance evaluation single-blind sample program is being developed from Hanford Site waste tanks. This project is known as the Sample Exchange Evaluation (SEE) Program. The SEE Program was established to meet customer concerns of having a "highly radioactive" sample in a matrix similar to tank waste. Presently, no government or commercial programs of this type are available. Hanford Performance Evaluation Program is administered by the Waste Management Laboratory Operations Program Management group.

Washington State requires all in-state laboratories performing water quality analyses to be accredited laboratories and to participate in the Environmental Monitoring Systems Laboratory-Cincinnati Semi-Annual Water Pollution Study Program sponsored by the EPA. The laboratory is a DOE (government) laboratory performing mixed and hazardous waste analyses, but it does participate in this EPA, Region 10, coordinated performance evaluation program.

The EPA and Washington State require laboratories analyzing samples from CERCLA-Superfund sites to participate in the Contract Laboratory Program Quarterly Blind Performance Evaluation Program. The laboratory is a DOE (government) laboratory and cannot be a contract laboratory, but will participate in this performance evaluation program until a more suitable matrix sample (standard) program is made available.

SAS participates in one or more of the following blind QC standard or performance evaluation programs:

- USEPA Office of Solid Waste and Emergency Response, CLP - Las Vegas Quarterly Blind Inorganic/Organic Performance Evaluation

- USEPA Environmental Monitoring Systems Laboratory-Cincinnati Semi-Annual Inorganic/Organic Water Pollution Performance Evaluation Studies

- USEPA Office of Research and Development, National Exposure Research Laboratory (NERL), formerly Environmental Monitoring Systems Laboratory (EMSL), - Las Vegas Radiological Performance Evaluation Studies

- DOE Environmental Measurements Laboratory - New York Semi-Annual Radiological Quality Assessment Program

- DOE Performance Demonstration Program (PDP), Transuranic Waste Characterization (TRU) Program for testing and analytical facilities (see CAO-94-1010, Section 2.3 [when applicable]) 
- WHC/Pacific Northwest National Laboratory Sample Exchange Evaluation Program-Richland Pilot Radiological/Inorganic Sample Exchange Performance Evaluation Program

- Laboratory QA Blind Studies (for example, ERA, APG, as determined).

Where applicable, results are reported via hard copy to the responsible technical manager/lead, sample preparation manager, and Hanford Performance Evaluation Program (HPEP) coordinator. Summary PE results are reported to management in the QA/QC status report by a distribution list compiled from the Hanford Analytical Laboratory Operations organization chart.

All noted deficiencies shall be investigated, resolved, and documented by the appropriate laboratory personnel. All documentation of corrective action shall be maintained in accordance with program protocol and tracked by DTS for water pollution and quarterly blinds. In addition, Hanford Analytical Laboratory Operations (HALO) program management is responsible for establishing and maintaining a database for tracking and trending concerns from the tracking and trending of the HPEP program.

\subsection{DATA QUALITY ASSESSMENTSAREVILWS}

Data quality assessments (reviews) are independent evaluations of the data reported to a client. They are used to assess the degree of compliance to client data quality requirements. These assessments may be organizationally or programmatically driven. The quality assurance officer staff should perform these assessments whenever practical. SAS performs a data quality review as required by the program or client on each data package. The assessment is a random review that checks 10 percent to 100 percent of the data reported. The objective is to determine if the requirements of the TSAP or other customer work-authorizing document are adequate and enough information and documentation exist within a given data package to support an assessment of its quality. Furthermore, the degree of conformance to client data quality requirements shall be evaluated and documented. Some issues that can be used to improve the data report quality but do not affect the data reported will be resolved through continuous quality improvements.

\subsection{EXTERNAL AUDITS OR ASSESSMENTS}

External auditst or assessments of the laboratory are performed by agencies or groups that are not under the control of laboratory management. These include regulators (for example, EPA, Ecology, Washington State Department of Health), clients, and the DOE. External audits may consist of inspections, interviews, and/or evaluations that focus on the laboratory's ability to meet client, program, and/or regulatory requirements (that is, Facility Evaluation Board [FEB] and Integrated Contractor Audit Team [ICAT]). Laboratory management shall be responsible for initiating, tracking, following up on, and documenting all corrective actions that are required as a result of external audits. 
HNF-SD-WM-QAPP-034 Rev. 3

\subsection{INSTRUMENT PREVENTIVE MAINTENANCE}

Preventive maintenance of laboratory instrumentation helps to ensure its availability for analytical measurements by reducing downtime and avoiding inconsistencies in instrument performance.

Instrument preventive maintenance is performed in accordance with a schedule thich is based on manufacturer's recommendations, instrument performance history, and operating instrument or tsystem characteristics and usage. Most laboratory instruments require some minor maintenance activities such as cleaning or other adjustments performed by the analyst as part of its setup or operation. The chemist consults the instrument manuals for the manufacturer's guidelines regarding daily setup, maintenance, and operation. Each instrument shall have a logbook to record maintenance events with date and name of the personnel performing the maintenance.

The following items should be considered for inclusion:

- Routine inspections (for example, daily, weekly, or as needed) should be based on the manufacturer's recommendations or instrument preventive maintenance schedules where practical and performed by the responsible analyst, and followed by corrective actions, if required. Anomalies should be noted individually in a logbook, on a record sheet, or in an electronic record system, which will be kept next to the instrument.

- Significant corrective actiontst for the M\&TE or instrument should be documented in a logbook, on a record sheet, or in an electronic record system. The notation should include a description of the corrective action, the date performed, and the initials of the person who performed the corrective action.

- Instrument maintenance should be performed by appropriate personnel. This could include services provided through an external maintenance contract.

- Instrument maintenance and repair documentation should be reviewed annually.

- Spare parts inventories may be items that are consumable day to day or-known-to-fail parts maintained at the discretion of the cognizant scientist.

Alternatives to spare parts inventories that help ensure minimal loss of analytical capacity include the following:

- Overnight parts availability from suppliers

- Availability of back-up instrumentation (see "Redundant Capacity" betow)

- Vendor service contracts

- Redundant capacity. 
. For most technologies, SAS has multiple units of equivalent instruments available. Also, the Waste Sampling and Characterization Facility (WSCF), 222-S Laboratory, and/or Pacific

Northwest National Laboratory have similar instrumentation in many areas. Both these resources can minimize the impact of downtime required for instrument maintenance. A recommended laboratory instrument preventive maintenance plan is provided in Table 15-1. 
Table 15-1. Recommended Preventive Maintenance Frequency for Specific Instruments. (3 sheets)

\begin{tabular}{|c|c|}
\hline Instruments & Frequency \\
\hline $\begin{array}{l}\text { Atomic Absorption (AA) Spectrophotometer } \\
\text { Inspect nebulizer } \\
\text { Inspect spectrophotometer quartz windows } \\
\text { Check burner head; check tubing, pump, and lamps } \\
\text { Check O rings } \\
\text { Fine tune wavelength; check optics } \\
\text { Check electronics }\end{array}$ & $\begin{array}{l}\text { Daily } \\
\text { Daily } \\
\text { Daily } \\
\text { Daily } \\
\text { Semiannually } \\
\text { Semiannually }\end{array}$ \\
\hline $\begin{array}{l}\text { Furnace AA Spectrophotometer } \\
\text { Check graphite tubes } \\
\text { Flush autosampler tubing } \\
\text { Replace graphite electrodes } \\
\text { Clean furnace housing and injector tip } \\
\text { Check electronics }\end{array}$ & $\begin{array}{l}\text { Daily } \\
\text { Daily } \\
\text { Annually } \\
\text { Daily } \\
\text { Annually }\end{array}$ \\
\hline $\begin{array}{l}\text { Cold Vapor and Hydride AA. Spectrophotometer } \\
\text { Check flushing tubing (automated systems) } \\
\text { Check absorption cell for vitrification } \\
\text { Replace or clean quartz cell } \\
\text { Check electronics }\end{array}$ & $\begin{array}{l}\text { Daily } \\
\text { Daily } \\
\text { As needed } \\
\text { Semiannually or as needed }\end{array}$ \\
\hline $\begin{array}{l}\text { Inductively Coupled Plasma Spectrometer (ICP) } \\
\text { Inspect torch } \\
\text { Clean nebulizer and spray chamber } \\
\text { Clean fan filters } \\
\text { Check peristaltic pump tubing and vacuum pump oil } \\
\text { Check optical system } \\
\text { Check water lines, torch compartment, and gases } \\
\text { Check electronics (for example, voltages, waveforms) } \\
\text { Check wavelength calibration and adjust as needed } \\
\text { Run interference (interelement) standard }\end{array}$ & $\begin{array}{l}\text { Semiannually } \\
\text { Weekly } \\
\text { Semiannually } \\
\text { Semiannually } \\
\text { Semiannually } \\
\text { Semiannually } \\
\text { Semiannually } \\
\text { Semiannually } \\
\text { Daily }\end{array}$ \\
\hline $\begin{array}{l}\text { Gas Chromatograph (GC) General } \\
\text { Check septa, cylinder gas pressure, oxygen/moisture traps } \\
\text { Bake out injector body } \\
\text { Check electronics (for example, voltages, waveforms) } \\
\text { Check GC temperature calibrations (injector, oven, detector) } \\
\text { Columns (change glass sleeve inserts, shorten ends of columns, change glass wool } \\
\text { plugs, check for leaks or replace) } \\
\text { Electron capture detector, wipe tests } \\
\text { Electron Capture Detector }\end{array}$ & \begin{tabular}{|l} 
Daily \\
Daily \\
Annually \\
Semiannually \\
As needed \\
Semiannually
\end{tabular} \\
\hline $\begin{array}{l}\text { Hydrogen cleaning } \\
\text { Retumed to factory for cleaning and refoil }\end{array}$ & As needed \\
\hline
\end{tabular}


Table 15-1. Recommended Preventive Maintenance Frequency for Specific Instruments. (3 sheets)

\begin{tabular}{|c|c|}
\hline Instruments & Frequency \\
\hline $\begin{array}{l}\text { GC/Mass Spectrometer } \\
\text { Replace vacuum pump oil and change desiccant, } \\
\text { Check ion source and analyzer (dismantle, clean, and replace parts as needed) } \\
\text { Check mechanic (vacuum pumps, relays, gas pressures and flows) } \\
\text { Check mass calibration with FC-43 (perfluorotributylamine) } \\
\text { Purge and trap } \\
\text { Clean sparger } \\
\text { Change trap } \\
\text { Bake trap } \\
\text { Check purge flow } \\
\text { Check for leaks }\end{array}$ & $\begin{array}{l}\text { Semiannually } \\
\text { As needed } \\
\text { Daily } \\
\text { Daily } \\
\text { Daily } \\
\text { As needed } \\
\text { As needed } \\
\text { Daily } \\
\text { As needed } \\
\text { As needed }\end{array}$ \\
\hline $\begin{array}{l}\text { High Pressure Liquid Chromatography } \\
\text { Gas lines checked for leaks } \\
\text { Clean mobile phase flow system and detector flow cells with nitric acid } \\
\text { Check pump seals and valve assemblies } \\
\text { Check solvent filters }\end{array}$ & $\begin{array}{l}\text { Daily } \\
\text { Semiannually } \\
\text { Daily } \\
\text { Monthly }\end{array}$ \\
\hline $\begin{array}{l}\text { Auto Samplers } \\
\text { Auto-injector or autovial } \\
\text { Check needles and tubing } \\
\text { Clean }\end{array}$ & $\begin{array}{l}\text { Daily } \\
\text { As needed } \\
\text { As needed }\end{array}$ \\
\hline Total Organic Carbon Analyzer & Annually \\
\hline $\begin{array}{c}\text { Uranium Fluorometry } \\
\text { Laser source }\end{array}$ & $\begin{array}{l}\text { As needed from quality } \\
\text { control check }\end{array}$ \\
\hline $\begin{array}{l}\text { Gas Proportional Counters } \\
\text { Clean autosample chamber }\end{array}$ & Monthly \\
\hline $\begin{array}{l}\text { Alpha Energy Analysis } \\
\text { Clean and inspect } \\
\text { Check microamps and verify voltage settings } \\
\text { Check peak channel } \\
\text { Check Detector resolution }\end{array}$ & $\begin{array}{l}\text { Monthly } \\
\text { Monthly } \\
\text { Monthly } \\
\text { Monthly }\end{array}$ \\
\hline $\begin{array}{l}\text { Gamma Energy Analyzer-Check and inspect } \\
\text { Pole zero } \\
\text { Lower level discriminator } \\
\text { Time constant } \\
\text { Amplifier's fine gain for energy/channel setting } \\
\text { Check Detector resolution }\end{array}$ & $\begin{array}{l}\text { Monthly } \\
\text { Monthily } \\
\text { Monthly } \\
\text { Monthly } \\
\text { Monthly }\end{array}$ \\
\hline $\begin{array}{l}\text { Liquid Scintillation Counter } \\
\text { Internal standard source } \\
\text { Clean and check autosampler and sampler well } \\
\text { Clean and inspect }\end{array}$ & $\begin{array}{l}\text { As needed } \\
\text { Semiannually } \\
\text { Monthly }\end{array}$ \\
\hline Differential Scanning Calorimetry & Annually \\
\hline Thermogravimetric Analyzer & Annually \\
\hline
\end{tabular}


HNF-SD-WM-QAPP-034 Rev. 3

Table 15-1. Recommended Preventive Maintenance Frequency for Specific Instruments. (3 sheets)

\begin{tabular}{|l|l|}
\hline \multicolumn{1}{|c|}{ Instruments } & \multicolumn{1}{c|}{ Frequency } \\
\hline pH Meter & Annually \\
\hline Thermometers & Annually \\
\hline $\begin{array}{l}\text { Analytical Balance } \\
\text { Cleaning and calibration }\end{array}$ & Annually \\
\hline Density Meter & Annually \\
\hline Ultraviolet/Nisible Spectrophotometer & Annually \\
\hline
\end{tabular}


HNF-SD-WM-QAPP-034 Rev. 3

This page intentionally left blank. 


\subsection{CORRECTIVE ACTION AND QUALITY IMPROVEMENT}

The corrective action process consists of identifying a deficiency, tracking and trending, using a graded approach via risk evaluation, root cause analyses, Price-Anderson screening, subject code, trend analysis, determining corrective action, and documentation. The laboratory will implement portions of the requirements of HNF-MP-599, Project Hanford Quality Assurance Program Description, Part 2, Section 2.0, "Quality Improvement" (Beyers and Grier 1997). This process also implements HNF-PRO-052, Corrective Action Management (CAM), and WMH-200, Section 1.3, "Corrective Action Management."

A deficiency is an inclusive term applied to adverse conditions, deviations, failures, malfunctions, defective items, nonconformances, or noncompliances in activities affecting quality, safety, health, operability, or the environment.

\subsection{INITIATION OF CORRECTIVE ACTION}

Examples of conditions (deficiencies) where corrective actions shall be implemented include the following:

- Documentation errors

- Adverse trends in the analysis of standards and blanks (refer to specific QC criteria on corrective action in Section 11.0)

- Failure to comply with approved procedures

- Failure to follow the preventive maintenance program

- Failures in the instrument systems

- Failures in performance evaluation sample analysis

- Non-compliance issues identified by audits, surveillances and assessments

- Validation and/or verification issues negatively impacting reported results

- Failure to follow client analytical requests and/or data quality objectives (DQOs). This condition may be addressed as part of the narrative in the data package report.

- Misidentification or mishandling of samples.

Items that do not meet the definition of deficiency include the following and will not be tracked in the DTS:

- Routine maintenance tasks, including preventive maintenance replacements of seals, gaskets, fluids, lighting fixtures, and so forth.

- Information that pertains to violations of general civil conduct under public law, such as harassment, sexual misconduct, assault, traffic citations, drug abuse, and so forth.

- Employee concerns, unless part of a report meeting other criteria under the definition of deficiency. 
- Equipment or instrument failures caused by a management decision to "run to failure" or where the equipment or instrument fails to perform beyond its design capacity.

\subsection{EVALUATING IMPACT AND EVENT INVESTIGATION}

Management shall be responsible for event investigations and impact determinations. Event investigation management policy is documented in the WMH-200, Section 2.6, "Occurrence Categorization, Notification, and Reporting," and Section 1.3, on "Corrective Action Management." The corrective-action processes describe the provisions for determining the significance of the problem and taking effective corrective action based on the potential impact on the data quality. The process can be initiated by submitting a fact sheet, critique, unusual occurrence report, nonconformance report, or letter of observation.

A quality assurance "letter of observation" is an internal corrective action program. (Refer to WMH-310, Section 8.1." This program allows for quick and efficient resolution of qualityaffecting observations that do not require external tracking. This procedure includes identification, notification, evaluation, documentation, and resolution of the quality-affecting issue. The key to this program is quick efficient internal corrective action, minimizing cost, and increasing productivity. The tracking is performed by the laboratory QA officer and the trending and statistical information is reported in the monthly QA/QC status report An NCR is written if the graded approach review indicates that the impact has involved customer issues (that is, a data package was reported with a procedure that used but not approved or agreed on by the customer). Observations are typically used for internal corrective actions when the concern was identified prior tobefore the final data package release.

When certain QC results fail to meet the control limits, the responsible chemist or manager is required to override data entry to the LABCORE (refer to Chapter 11, "Quality Control"). Sample results from the-batch associated with this failing $\mathrm{QC}$ result shall be entered. In this case, sample results shall be flagged with appropriate qualifiers or documented in the narrative. Samples may need to be reanalyzed, depending on the types of QC failure.

Implementation of corrective actions shall be verified as appropriate. When corrective actions involve a measurement system, the corrective response will be fulfilled when the flagging is complete and the narrative documents the $\mathrm{QC}$ problems.

To promote timeliness and efficiency in the laboratory, corrective actions are made at the lowest management level possible.

- First-Level Managers shall review all reports of problems generated in their respective units. The first-level managers are responsible for resolving problems and ensuring that $\mathrm{QA} / \mathrm{QC}$ objectives are met.

- Responsible Scientists/Engineers shall assist unit managers in the resolution of problems relating to their area of responsibility. 
- Section Managers provide overall coordination of laboratory operations, and are responsible for corrective action and closure in their areas of responsibility.

- Operations Support Manager is responsible for the overall tracking of corrective actions per the Hanford Action Tracking System (HATS) database.

- Laboratory Quality Assurance Officer or QA/QC representatives are responsible for overseeing quality-related problems and issuing (that is, letters of observation, performance audit corrective actions, NCRs as necessary).

\subsection{ROOT CAUSE ANALYSIS}

The extent of the root cause analysis shall be commensurate with the importance or significance of the problem (see HINF-PRO-052 for reference).

A graded approach shall be used for all quality-affecting activities at the 222-S Laboratory. The graded approach takes into account such factors as the following:

- Level of risk to workers, facilities, and the environment, and impact associated with a failure or deficiency.

- Age, status and condition of a facility, process, or an item.

- History of problems at a facility, with a process, method, or item.

- Current safety documentation.

- Complexity of products or activities involved.

- Control of potential project delays and costs if failures or deficiencies occur.

\subsection{RECURRING CONDITIONS ADVERSE TO QUALITY}

These processes are established in the procedure used to correct the problem. The measures to eliminate or minimize recurrence of quality problems shall be established using the following provisions. These determinatios shall include, but not be limited to, the following.

- Determine the events leading to the adverse condition.

- Understand the technical and work activities associated with the quality problem.

- Ascertain the quality problem's generic implications. 
HNF-SD-WM-QAPP-034 Rev. 3

- Determine the extent to which similar quality problems or their precursors have been recognized.

- Determine the effectiveness of any corrective actions that were taken.

- Determine the impacts on the completed work.

- Recommend actions that can be taken by the responsible organization to preclude recurrence.

- Determine if the work associated with the activity should be stopped.

\subsection{TREND ANALYSIS}

Where possible, analysis of quality-related information shall include identifying common work processes for quality problems, conducting cause-and-effect analyses, and determining effective corrective and preventive actions from internal sources, including other DOE facilities or sites.

| Quality-related information to be analyzed shall include, but is not limited to, the following, as appropriate:

- Performance data

- Audit reports

- Surveillance reports

- Nonconformance reports

- Quality-related information from external sources (not limited to one type of work, one facility, or one contractor)

- Performance indicators.

- Failure rates (for example, QC reruns, pareto charts).

Corrective Action Management Systems data located in the DTS database shall be analyzed to identify trends that adversely taffect quality and to identify opportunities to improve processes and items. Statistically significant trends shall be promptly reported to senior management. 


\subsection{LESSONS-LEARNED EVALUATIONS}

Lessons-learned evaluations (LLE) shall be performed to determine if the circumstances that caused a condition in one area could be present elsewhere, so that these conditions can be avoided or prevented.

Detailed instructions for performing and distributing LLE are contained in HNF-PRO-067, Managing Lessons Learned, and WMH-200, Section 1.1 1, "Lessons Learned Administration."

\subsection{CONTINUOUS QUALITY IMPROVEMENT}

Quality improvement is a continuous process and is-designed to reduce the variability of every process that influences the quality of the product. This concept is used throughout this quality assurance program. Laboratory activities, analytical measurements and results, and QA/QC activities are documented and are-traceable for evaluation. For example, management or nonsupervisory personnel can analyze the performance indicators, control charting, corrective actions, or assessments to identify actions as a means of continuous quality improvement. In addition, information obtained from LLEs, DTSs, or peer reviews also areis used for management planning and problem prevention as a means of continuous quality improvement. Quality improvement generally results from activities that do the following:

- Prevent or minimize problems during the planning and implementation of sampling and analysis activities that may affect the quality of the results.

- Detect and correct the problems.

- Review existing performance and identify opportunities for quality improvement.

\subsection{CONTROL OF NONCONFORMANCES}

Controls shall be implemented to prevent the inadvertent use of samples, materials, parts, or components that do not conform to requirements. These measures shall include, as appropriate, procedures for identifying, documenting, evaluating, segregating (where practical, disposing of, and notifying affected organizations. Refer to Chapter 6, "Sample Custody and Handling;" Chapter 18, "Procurement Controls;" and-HNF-MP-599, Project Hanford Quality Assurance Program Description (Beyers and Grier 1997); and HNF-PRO-298, Control of Non-Conforming Items. 
TABLE 16-1. Recommended Field Laboratory QA/QC Requirements.

\begin{tabular}{|c|c|}
\hline Quality Control Required & Frequency and Acceptance Criteria \\
\hline Initial calibration & $\begin{array}{l}\text { Daily/linear correlation }>0.995 \\
\text { or as needed, based on calibration check performance }\end{array}$ \\
\hline Calibration check & $\begin{array}{l}\text { Immediately following initial calib. and every } 10 \text { analysis } \\
\text { thereafter. } \% \text { Recovery within }+/-20 \% \text { true value }\end{array}$ \\
\hline Spike sample (predigestion) & $\begin{array}{l}\text { One per } 20 \text { samples. } \% \text { Recovery within } 25 \% \text { true value } \\
\text { unless sample concentration }>4 \mathrm{X} \text { spike concentration }\end{array}$ \\
\hline Matrix spike & One per 20 samples. $\%$ Recovery within $25 \%$ true value \\
\hline Duplicate sample & $\begin{array}{l}\text { One per } 20 \text { samples. } \% \text { Recovery }<=20 \% \text { for waters, } \\
<=35 \% \text { for soils }\end{array}$ \\
\hline $\begin{array}{l}\text { Internal standards, laboratory } \\
\text { control samples }\end{array}$ & $\begin{array}{l}\text { One per } 20 \text { samples/matrix/batch. } \\
\text { Results within } 80 \text { to } 120 \% \text { of true values }\end{array}$ \\
\hline Method blanks & $\begin{array}{l}\text { One per } 20 \text { samples/matrix/batch. } \\
\text { Results less than minimum detectable limits }\end{array}$ \\
\hline Minimum detection limits & $\begin{array}{l}\text { Reported for each sample, analyte, matrix, results must } \\
\text { meet DQO, work-plan detection limits. Conducted on } \\
\text { standard matrix material during method evaluation. } \\
\text { Project-specific matrix MDLs will be conducted as } \\
\text { specified by client }\end{array}$ \\
\hline
\end{tabular}

NOTE: The DQO process and/or the statements of work, work plans, letter of instructions will govern the QA/QC requirements.

DQO $=$ data quality objective

$\mathrm{MOL}=$ minimum detection limit 


\subsection{QUALITY ASSURANCE REPORTS}

A formal mechanism for reporting to management the status of the QA program shall be established and implemented. QA reports to management shall be issued annually, at a minimum.

The QA/QC officer shall prepare a status report to management using the established format of an internal memo. The report is issued to the manager and distributed to the appropriate personnel. The report shall identify (if applicable for the reporting period), the following:

- The reporting period

- Individual(s) who will receive the report

- Individual(s) responsible for preparing the report

- Topics to be discussed - at a minimum, reports to management on QA activities shall include a summary of the results onof the following:

- Performance evaluation audits

- Technical system audits

- Management system audits

- External audits, assessments, and surveillance activities

- Data quality audits and validation audits (validation audits are typically performed by the customer) because of limited QA staffing)

- Regulatory compliance issues

- Assessment of data quality, accuracy, precision, and method detection limits are summarized in data packages reported to the customer

- Major data-quality problems identified in data-quality audits will be summarized in the QA status report.

- Precision and accuracy of standards via control chart reevaluation requests will be reported in the QA status report.

- Method detection limits add no value by including them in the management status report. They are already in each data package. Method detection limit problems will be reported to management.

- QA and QC problems and recommended solutions 
- Internal QA corrective actions and status identified by the Letter of Observation program; HATS items are summarized and reported to management separately from the QA status report

1. - Recommended changes to the QA program. 


\subsection{DATA VALIDATION}

The project DQO/DQR requirements must be defined in the specific QAPP, TSAP, or other work-authorization document. In cases where data must be validated, a specific set of acceptance criteria must be defined that provides assurance that the data generated are adequate for their intended use. The acceptance criteria reflect the requirements generated during the DQO planning process.

Data validation is the systematic review of analytical data from samples and their associated laboratory QC samples. Data validation is the assessment (review) that determines whether the proper analyses were performed and recorded as defined by the DQO/DQR or work-authorization document, -the correct documentation is present to verify that the controls in the laboratory were performed, and the data are defensible to the requirements of the DQO/DQR or workauthorization documents. The purpose of the review is to make determinations concerning data quality and data limitations to assist the user in avoiding inappropriate use of the data, while not precluding any consideration of the data at all. This is accomplished through documentation and use of data qualifiers. Typical data qualifiers used are the following:

- U The material was analyzed for, but was not detected. The associated value is the MDL.

- UJ The material was analyzed for, but was not detected. The MDL is an estimated quantity.

- $\mathrm{J}$ The associated value is an estimated quantity.

- $\mathrm{R}$ The data are unusable.

- O Data had no problems.

- X Data qualified because of minor problems.

- M Data qualified because of major problems/some data may be usable.

Data validation is covered in administrative procedure HNF-CM-5-4, Section 3.25, "Data Validation for High Level RCRA/CERCLA Radiochemical Analyses," and supporting document WHC-SD-EN-SPP-002, DATA Validation Procedures for Chemical Analyses (Pool 1993). The following areas are typically assessed in validation procedures:

- Holding times

- Preservative methods and container types

- Minimum required sample size, for analysis

- Calibration criteria and requirements including initial and continuing 
- Methods blank, instrument blanks, preparation blanks and/or background requirements

- Matrix spike recoveries and/or tracer or carrier yields

- Duplicates

- Laboratory control samples

- Identification of analytes(s) of interest

- Interferences

- Quantitation criteria

- Instrument performance and counter efficiency

- Criteria for validation of detection limits

- Criteria for accuracy and precision assessment.

For organic compound analyses, the following areas should be addressed in addition to the previously stated items:

- Surrogate recoveries for organic compounds used to assess accuracy

- Tuning (used for GC/MS only)

- Lower area limits for minimum detection

- Chromatograms showing manual integration of baselines and spectra (including tentatively identified compounds)

- Instrument carry-over between analytical runs.

Validation of ICP should include criteria for evaluating interelement correction factors and how interferences shall be validated.

\subsection{PLANNING CONSIDERATIONS}

A common problem is that validation and assessment of data are planned after the fact, or after the data are collected. This sometimes results in the deliverables from the sampling and laboratory analysis being insufficient (for example, disorganized and inadequately documented) for complete assessment of the data. The cost of validating poorly planned and insufficient data reports wiltincreases the original laboratory analysis costs. The problem is compounded when 
methods other than the CLP protocols are specified. The CLP methods have published validation criteria, while other methods do not. The QC differs between the various methods. For example, the most recent CLP GC/MS method for semivolatile organic compounds requires that all but five compounds have minimum response factors, while SW-846 methods use four compounds to verify minimum response. This difference may be significant in the final outcome of the validation process for semivolatile organic compounds. (Refer to HASQARD, Volume 4, Chapter 9 for additional information on "assessment and validation"t of data.)

\subsection{DATA USABILITY}

-Data are usable if they meet the intended end use as specified in the DQO process. However, many validation procedures do not address data use. The following important facts need to be understood about many validation and assessment procedures.

- Method deficiencies may be observed, but may not result in data being qualified (flagged as estimated or rejected).

- Data may be qualified and still be usable.

- Many published validation procedures do not address all aspects of data review. 


\subsection{PROCUREMENT CONTROLS}

SAS follows HNF-SD-WM-QAPP-036, Section 3.2.3, HNF-MP-599, Project Hanford Quality Assurance Program Description, Section 7, "Procurement", and Section 8, "Inspection and Acceptance Testing", as well as HNF-PRO-268, Control of Purchased Items and Services, to ensure the procurement process is documented and performed according to the Sitewide requirements.

\subsection{PURCHASE OF COMMERCIAL CHEMICAL STANDARDS}

I Chemicals are purchased at the grade and purity appropriate for their intended use. Generally, reagent grade or better is purchased for laboratory use.

| Vendor-supplied chemical standards must be shipped with a certificate of quality showing analytical value of the standard plus traceability to NIST or other proof of quality where traceability to NIST is not available.

\subsection{RECIEVING INSPECTION}

Recieving inspection shall be performed in accordance with HNF-PRO-283, Control of Inspections.

\subsection{DENTIFIED NONCONFORMANCES}

All items determined to be nonconforming during recieving inspection, and all items with proposed dispositions of "use-as-is" or "repair" shall receive the same level of review and approval as nonnonconformancces identified in HNF-PRO-298, Nonconforning Item Reporting and Control.

\subsection{CONTROL OF SUSPECT COUNTERFEIT ITEMS}

Items that are suspected to be counterfeit are handled in accordance with HNF-PRO-301, Control of Suspect Counterfeit Items. 
HNF-SD-WM-QAPP-034 Rev. 3

This page intentionally left blank. 


\subsection{REFERENCES}

10 CFR 830.120, 1994, "Quality Assurance Requirements," Title 10, Code of Federal Regulations, as amended

29 CFR 1910, 1992, "Occupational Safety and Health Standards," Title 29, Code of Federal Regulations, as amended.

40 CFR 136, 1994, "Guidelines Establishing Test Procedures for the Analysis of Pollutants," Title 40, Code of Federal Regulations, as amended

49 CFR 100 to 179, 1991, "DOT Hazardous Materials Regulations," Code of Federal Regulations, as amended.

ASTM, 1977, Standard Practices for Sampling Water, ASTM-3370-76, American Society for Testing and Material, Philadelphia, Pennsylvania.

ASTM, 1980, Anmual Book of ASTM Standards, Section E-29, "Standard Practice for Using Significant Digits in Test Data to Determine Conformance with Specifications,"American Society for Testing and Materials, Philadelphia, Pennsylvania.

ASTM, 1983, Sampling Surface Soils for Radionuclides, ASTM-C-998-83, American Society for Testing and Material, Philadelphia, Pennsylvania.

, TRU Waste Isolation Pilot Program Quality Assurance Project Plan, HNF-2599, for Fluor Daniel Hanford, Inc., Richland, Washington.

, HNF-IP-1128, Numatec Hanford Special Analytical Support Procedures Manual, for Fluor Daniel Hanford, Inc., Richland, Washington.

WHC-SP-1 131, Westinghouse Hanford Company, Richland, Washington.

1994, Contract Laboratory Program Statement of Work, Richland, Washington.

Beyers, S. A. and G. W. Grier, 1997, Project Hanford Quality Assurance Program Description, HNF-MP-599, Rev. 1, Fluor Daniel Hanford, Inc., Richland, Washington.

Clean Air Act of 1977, 42USC 7401 et seq.

Clean Water Act of 1977, 33 USC 1251 et seq.

Comprehensive Environmental Response, Compensation, and Liability Act of 1980,42 USC 960 I et seq. 
Currie, L. A., 1968, "Limits for Qualitative Detection and Quantitative Determination," Analytical Chemistry, 40(3):586-593.

DOE, 1987, Records Program Management, DOE Order 1324.5B, U.S. Department of Energy, Washington, D.C.

DOE, 1987, Environmental Survey Manual, DOE/EH-0053, U.S. Department of Energy, Washington, D.C.

DOE, 1988, Radioactive Waste Management, DOE Order 5820.2A, U.S. Department of Energy, Washington, D.C.

DOE, 1991, Quality Assurance, DOE Order 5700.6C, U.S. Department of Energy, Washington, D.C.

DOE, 1992, Methods for Evaluating Environmental and Waste Management Samples, DOE/EM-0089T, U.S. Department of Energy, Washington, D.C.

DOE, 1992, EML Procedures Manual, 27th Edition, U.S. Department of Energy, Washington, D.C.

DOE/RL, 1995, Hanford Analytical Services Quality Assurance Requirements Document, DOE/RL-96-68, Department of Energy, Richland, Washington.

EPA, 1977, Sampling of Water and Wastewater, U.S. Environmental Protection Agency, Washington, D.C.

EPA, 1980, Guidelines and Specifications for Preparing Quality Assurance Program Plans, EPA QAMS-004/80, U.S. Environmental Protection Agency, Washington, D.C.

EPA, 1980, Interim Guidelines and Specifications for Preparing Quality Assurance Project Plans, EPA QAMS-005/80, U.S. Environmental Protection Agency, Washington, D.C.

EPA, 1985, Compendium of Superfund Field Operations Manual, U.S. Environmental Protection Agency, Washington, D.C.

EPA, 1989, Soil Sampling Quality Assurance User's Guide, U.S. Environmental Protection Agency, Washington, D.C.

EPA, 1991, Representative Sampling Guidance, Vol. 1, "Soil," U.S. Environmental Protection Agency, Washington, D.C.

EPA, 1.992, Test Methods for Evaluating Solid Waste: Physical/Chemical Methods, SW-846, U.S. Environmental Protection Agency, Office of Solid Waste and Emergency Response, Washington, D.C. 
EPA, 1992, Test Methods for Evaluating Solid Waste: Physical/Chemical Methods, SW-846, U.S. Environmental Protection Agency, Office of Solid Waste and Emergency Response, Washington, D.C.

Jacobsen, P. H., 1998, Quality Assurance Program Plan, HNF-SD-WM-QAPP-036, Rev. 1, Waste Management Federal Services of Hanford, Inc. for Fluor Daniel Hanford, Inc., Richland, Washington.

Keith, L. H., 1991, Environmental Sampling and Analysis, A Practical Guide, Lewis Publishers, Chelsea, Michigan.

Markel, L. P., 1996, Hanford Performance Evaluation Program, WHC.SD-QA-QAPP-002, Rev. 1, Westinghouse Hanford Company, Richland, Washington.

Moss, S. S., 1989, Quality Assurance Project Plan for the Chemical Analysis of Highly Radioactive Mixed Waste Samples in Support of Environmental Activities on the Hanford Site, WHC-SD-QAPP-002, Rev. 0, Westinghouse Hanford Company, Richland, Washington.

Myers, M. L., 1998, Special Analytical Support Training Plan, HNF-3275, Waste Mangement Federal Services of Hanford, Inc., for Fluor Daniel Hanford, Inc., Richland, Washington.

Occupational Safety and Health Act of 1970, 21 USC 651 et seq.

Pool, K. N., 1993, DATA Validation Procedures for Chemical Analyses, WHC-SD-EN-SPP-002, Rev. 2, Westinghouse Hanford Company, Richland, Washington.

Resource Conservation and Recovery Act of 1976, 42 USC 6901 et seq.

Roberts, J. M., 1996, Quality Assurance Program and Implementation Plan, WHC-SP-1131, Rev. 1, Westinghouse Hanford Company, Richland, Washington.

Rich, H. S., 1995, Configuration Management Plan for LABCORE Program, HNF-SD-WM-CM-002, Westinghouse Hanford Company, Richland, Washington.

WAC-173-303, "Dangerous Waste Regulations," Washington Administrative Code, as amended.

WAC-246-247, "Radiation Protection-Air Emissions," Washington Administrative Code, as amended.

Weaver, L. L., 1997, 222-S Laboratory Interim Safety Basis, HNF-SD-CP-ISB-002, Rev. 2, Waste Management Federal Services of Hanford, Inc., for Fluor Daniel Hanford Company, Richland, Washington. 


\subsection{HANFORD NUCLEAR FACILITY, HANFORD ANALYTICAL LABORATORY OPERATIONS PROCEDURES}

HNF-PRO-052, Corrective Action Management, Fluor Daniel Hanford, Inc, Richland, Washington.

HNF-PRO-067, Managing Lessons Learned, Fluor Daniel Hanford, Inc., Richland, Washington.

HNF-PRO-123, The Written Requisition Process, Fluor Daniel Hanford, Inc., Richland, Washington.

HNF-PRO-126, Processing the Release to Ship, Fluor Daniel Hanford, Inc., Richland, Washington.

HNF-PRO-167, Using the SAT Model For Training, Fluor Daniel Hanford, Inc., Richland, Washington.

HNF-PRO-168, Employee Training, Fluor Daniel Hanford, Inc.; Richland, Washington.

HNF-PRO-169, Assigning Training Requirements, Fluor Daniel Hanford, Inc., Richland, Washington.

HNF-PRO-170, Analyzing Training Requirements, Fluor Daniel Hanford, Inc., Richland, Washington.

HNF-PRO-171, Designing Training, Fluor Daniel Hanford, Inc., Richland, Washington.

HNF-PRO-172, Developing Training, Fluor Daniel Hanford, Inc., Richland, Washington.

HNF-PRO-173, Implementing Training, Fluor Daniel Hanford, Inc., Richland, Washington.

HNF-PRO-174, Evaluating Training, Fluor Daniel Hanford, Inc., Richland, Washington.

HNF-PRO-175, Developing a Qualification Program, Fluor Daniel Hanford, Inc., Richland, Washington.

HNF-PRO-176, Preparing Qualification Programs at Nuclear Facilities, Fluor Daniel Hanford, Inc., Richland, Washington.

HNF-PRO-177, Preparing Certification Programs at Nuclear Facilities, Fluor Daniel Hanford, Inc., Richland, Washington.

HNF-PRO-178, Qualifying Instructional Staff, Fluor Daniel Hanford, Inc., Richland, Washington. 
HNF-PRO-179, Obtaining Training Exceptions and Extensions, Fluor Daniel Hanford, Inc,, Richland, Washington.

HNF-PRO-208, Records Management, General, Fluor Daniel Hanford, Inc., Richland, Washington.

HNF-PRO-210, Records Management Program, Fluor Daniel Hanford, Inc., Richland, Washington.

HNF-PRO-211, Administrative Record, Fluor Daniel Hanford, Inc., Richland, Washington.

HNF-PRO-212, Public Information Repository, Fluor Daniel Hanford, Inc., Richland, Washington.

HNF-PRO-213, Record or Non-Record Material, Fluor Daniel Hanford,.Inc., Richland, Washington.

HINF-PRO-214, Record Inventory and Disposition Schedules, Fluor Daniel Hanford, Inc., Richland, Washington.

HNF-PRO-215, Records Storage, Retrieval and Destruction, Fluor Daniel Hanford, Inc., Richland, Washington.

HNF-PRO-216, Vital Records, Fluor Daniel Hanford, Inc., Richland, Washington.

HNF-PRO-222, Quality Assurance Records, Fluor Daniel Hanford, Inc., Richland, Washington.

HNF-PRO-223, Legal and Ethical Conduct, Fluor Daniel Hanford, Inc., Richland, Washington.

HNF-PRO-224, Document Control, Fluor Daniel Hanford, Inc., Richland, Washington.

HNF-PRO-225, Distributing Copy-Controlled Unclassified Documents, Fluor Daniel Hanford, Inc., Richland, Washington.

HNF-PRO-226, Classified Document Control, Fluor Daniel Hanford, Inc., Richland, Washington.

HINF-PRO-227, Engineering Document Identification, Fluor Daniel Hanford, Inc., Richland, Washington.

HNF-PRO-228, Central Files and Distribution of Non-Copy-Controlled Documents, Fluor Daniel Hanford, Inc., Richland, Washington.

HNF-PRO-229, Technical PR Standard, Fluor Daniel Hanford, Inc., Richland, Washington.

HNF-PRO-230, Controlling Field, Laboratory, and Facility Notebooks/Logbooks, Fluor Daniel Hanford, Inc., Richland, Washington. 
HNE-PRO-231, Correspondence and Commitment Control, Fluor Daniel Hanford, Inc., Richland, Washington.

HNF-PRO-232, Project and Task Document Management, Fluor Daniel Hanford, Inc., Richland, Washington.

HNF-PRO-233, Review and Approval of Documents, Fluor Daniel Hanford, Inc., Richland, Washington.

HNF-PRO-238, Need Title, Fluor Daniel Hanford, Inc., Richland, Washington.

HNF-PRO-246, Management Assessment, Fluor Daniel Hanford, Inc., Richland, Washington.

HNF-PRO-261, Quality Assurance Program Plans, Fluor Daniel Hanford, Inc., Richland, Washington.

HNF-PRO-268, Need Title, Fluor Daniel Hanford, Inc., Richland, Washington.

HNF-PRO-283, Control of Inspection, Fluor Daniel Hanford, Inc., Richland, Washington.

HNF-PRO-298, Control of Non-Conforming Items, Fluor Daniel Hanford, Inc., Richland, Washịgton.

HNF-PRO-301, Control of Suspect Counterfeit Items, Fluor Daniel Hanford, Inc., Richland, Washington.

HNF-PRO-309, Control of Computer Software, Fluor Daniel Hanford, Inc., Richland, Washington.

HNF-PRO-335, Use and Control of Purchasing Card (P Card), Fluor Daniel Hanford, Inc., Richland, Washington.

HNF-PRO-433, Small Job Development, Fluor Daniel Hanford, Inc., Richland, Washington.

HNF-PRO-440, Engineering Document Change Control Requirements, Fluor Daniel Hanford, Inc., Richland, Washington.

HNF-PRO-451, Regulated Substance Management, Fluor Daniel Hanford, Inc., Richland, Washington.

HNF-PRO-452, NEPA, SEPA, Cultural and Natural Resources, Fluor Daniel Hanford, Inc., Richland, Washington.

HNF-PRO-453, Environmental Notification and Reporting, Fluor Daniel Hanford, Inc., Richland, Washington. 
HNF-PRO-454, Inactive Waste Sites, Fluor Daniel Hanford, Inc., Richland, Washington.

HNF-PRO-455, Solid Waste Management, Fluor Daniel Hanford, Inc., Richland, Washington.

| HNF-PRO-456, Water Quality, Fluor Daniel Hanford, Inc., Richland, Washington.

HNF-PRO-457, Environmental Requirements Evaluation, Fluor Daniel Hanford, Inc., Richland, Washington.

HNF-PRO-458, Data Management, Fluor Daniel Hanford, Inc., Richland, Washington.

HNF-PRO-459, Environmental Training, Fluor Daniel Hanford, Inc., Richland, Washington.

HNF-PRO-462, Pollution Prevention, Fluor Daniel Hanford, Inc., Richland, Washington.

HNF-PRO-651, Surveillances, Fluor Daniel Hanford, Inc., Richland, Washington.

HNF-PRO-652, Corrective Action Requests, Fluor Daniel Hanford, Inc., Richland, Washington.

HNF-PRO-690, Applicable Environmental Statutes, Regulations, Fluor Daniel Hanford, Inc., Richland, Washington.

LC-100-400, Need Title, , Richland, Washington.

LC-400-002, Programmable Calculator Documentation and Coding Guidelines, Richland, Washington.

LC-400-003, Basic Coding and Documentation Guidelines, Richland, Washington.

LC-400-006, Spreadsheet Documentation Guidelines, Richland, Washington.

LO-090-405, Title, Publisher, City, State.

LO-090-450, Title, Publisher, City, State.

LO-100-107, Cubicle Housekeeping, Waste Disposal and Management, Richland, Washington.

LO-100-151, Segregate and Manage Solid Laboratory Waste, Richland, Washington.

LO-100-166, Manage Satellite Accumulation Area, 90-Day Accumulation Area, and the I Treatment, Storage, and Disposal (TSD) Units, Richland, Washington.

LO-100-400, SAS Waste Management, Need Company Name, Richland, Washington.

LO-110-128, Manage Laboratory Liquid Wastes, Richland, Washington. 
L0-120-101, Proper Labeling, Recertification of Chemicals, Standards, and Reagents, Richland, Washington.

LO-140-101, Routine Use and Quality Assurance for Analytical Balances at 222-S Laboratory, Richland, Washington.

LO-150-061, Criteria for the Recertification of Standards, Richland, Washington.

LO-150-135, Sample Disposal Criteria, Richland, Washington.

LO-150-172, Acceptance and Configuration Control of Instrument/Instrument Software, Richland, Washington.

LQ-150-001, Quality Control Charts/Statistical Process Control, Richland, Washington.

\subsection{WASTE MANAGEMENT FEDERAL SERVICES HANFORD. INC., POLICIES/PROCEDURES}

WMH-100, Waste Management Hanford Policies, Waste Management Federal Services, Hanford, Inc., Richland, Washington.

WMH-200, Waste Management Hanford Procedures, Waste Management Federal Services, Hanford, Inc., Richland, Washington.

WMH-3 10, Laboratories Administration, Waste Management Federal Services, Hanford, Inc., Richland, Washington.

\subsection{WESTINGHOUSE HANFORD COMPANY CONTROL MANUALS}

HNF-CM-1-3, Management Requirements and Procedures, Westinghouse Hanford Company, Richland, Washington.

HNF-CM-1-5, Standard Operating Practices, Westinghouse Hanford Company, Richland, Washington.

HNF-CM-4-2, Quality Assurance Manual, Westinghouse Hanford Company, Richland, Washington.

HNF-CM-5-4, Laboratories Administration, Westinghouse Hanford Company, Richland, Washington.

HNE-CM-7-5, Environmental Compliance, Westinghouse Hanford Company, Richland, Washington. 


\subsection{BIBLIOGRAPHY}

ASTM, 1995, Annual Book of ASTM Standards, Section 11, Water and Environmental Technology, Method E380, ASTM, Philadelphia, Pennsylvania.

David, Howard, 1984, Guidelines for Screening National Pollution Discharge Elimination System Permits.

Ecology, EPA, DOE, 1996, Hanford Federal Facility Agreement and Consent Order, as amended, Washington State Department of Ecology, U.S. Environmental Protection Agency, and U.S. Department of Energy, Olympia, Washington.

EPA, 1977, Environmental Protection Agency National Emission Standards for Hazardous Air Pollutants, U.S. Environmental Protection Agency, Office of General Enforcement, Washington, D.C.

EPA, 1991, USEPA Contract Laboratory Program, Statement of Work for Organics Analysis, OLM03. I, U.S. Environmental Protection Agency, Washington, D.C.

EPA, 1991, USEPA Contract Laboratory Program, Statement of Work for Inorganics Analysis, MM04.0, U.S. Environmental Protection Agency, Washington, D.C.

Moss, S. S., 1992, Quality Assurance Project Plan for the Chemical Analysis of Highly Radioactive Samples in Support of Environmental Activities on the Hanford Site, WHC-SD-CP-QAPP-002, Rev. 0, Westinghouse Hanford Company, Richland, Washington.

Moss, S. S., 1993, Quality Assurance Program Plan for Laboratory Analyses and Process Testing, WHC-SD-CP-QAPP-003, Rev. 1, Westinghouse Hanford Company, Richland, Washington.

Rosecrance, A., 1996, Environmental Testing \& Analysis, Volume 5, Number 4, The Target Group Inc., Burbank, California.

Schreiber, R. D., 1997, Fiscal Year 1997 Memorandum of Understanding for the TWRS Characterization Project, HNF-SD-WM-SD-023, Rev. 0, Lockheed Martin Hanford Corporation for Fluor Daniel Hanford, Inc., Richland, Washington.

Steel, R. G. D., and J. H. Toree, 1960, Principles and Procedures of Statistics, McGraw-Hill Inc., New York, New York. 
HNF-SD-WM-QAPP-034 Rev. 3

Winters, W. I., A. G. King, and T. C. Trible, 1996, Management of Data Quality for High-Level Waste Characterization, WHC-SA-3085-FP, Westinghouse Hanford Company, Richland, Washington 


\section{APPENDIX A - GLOSSARY}

accuracy

The degree of agreement of a measurement (or an average of measurements of the same thing), $\mathrm{X}$, with an accepted reference or true value, $T$, usually expressed as the difference between the two values, $\mathrm{X}-\mathrm{T}$, or the difference as a percentage of the reference or true value, $100(\mathrm{X}-\mathrm{T}) / \mathrm{T}$, and sometimes expressed as a ratio, X/T. Accuracy is A measure of the bias in a system.

analyst $\mathrm{A}$ person performing a measurement.

analyte The element, isotope, specie, or characteristic of a measurement.

anomalies Something different, abnormal, or peculiar, not easily classified.

assessment The act or instance of assessing (appraisal); the act of reviewing, inspecting, testing, checking, conducting surveillance, auditing, or otherwise determining and documenting whether items processes or services meet specified requirements.

The terms assessment and verification as used in DOE Order $5700.6 \mathrm{C}$ are synonymous; their use is determined by who is performing the work. Assessments are performed by or for senior management. Verifications are performed by the line organizations.

For data, assessment encompasses verification and validation. Data assessment (verification and/or validation) can be performed within the laboratory and/or by an independent review agency at the discretion of the client to the criteria of the project.

chemist/scientist

The person with a technical degree in chemistry or laboratory science who develops the specific analytical methods and completes the chemical compatibility assessment.

client The person or organization submitting work.

comparability Expresses the confidence with which one data set can be compared to another.

completeness A measure of the amount of valid data obtained from a measurement system compared to the amount that was expected to be obtained under correct normal conditions.

consensus standard A procedure, protocol, or guidance document issued by a professional organization based on extensive testing and peer review.

continuous quality A program that is designed to reduce the variability of every process improvement that influences the quality of a product. 
deviation Divergence from the original procedure that does not adversely impact the analyst's ability to meet the precision, accuracy, detection limit, selectivity, and quality control criteria of the procedure.

false negatives

A term that identifies the acceptance of a test or condition as false, when in fact it is true.

false positive A term that identifies the acceptance of a test or condition as true, when in fact it is false.

hazardous waste State listed wastes such as acids, carcinogenics, toxics, and so forth.

Instrument Detection The smallest signal above background noise that an instrument can Limit (IDL) detect reliably.

matrix The component or substrate (for example, surface water, drinking water) that contains the analyte of interest.

mixed waste State listed wastes with radiological contamination such as waste having both hazardous and radioactive components.

method generated waste Any waste generated and collected in satellite accumulation areas in the process of running an analytical method, including processes directed in an analytical method.

modification Changes to the character of a method, and thereby, potentially limits a method's capability to meet the originally stated precision, accuracy, detection limit, selectivity, and quality control criteria. Because the impact of such a modification cannot be ascertained before implementation, it must be demonstrated by application.

nonconformance

A deficiency in characteristic, documentation, or procedure that renders the quality of an item or activity unacceptable or indeterminate.

operator of the The person who has primary responsibility for running the processprocess generating the waste (for example, chemical technologist) or the person supervising the process. This is the person who has the authority to add waste to the container (for example, satellite accumulation area) or to direct waste to be added:

out-of-control A system is said to be out-of-control when it fails to meet (QC Failure) preselected performance criteria.

precision A measure of mutual agreement among individual measurements of the same property, usually under prescribed similar conditions. Precision is best expressed 
in terms of the standard deviation. Various measures of precision exist depending upon the "prescribed similar conditions."

preventive A program of instrument care based on scheduled activities and maintenance spare parts inventory designed to minimize instrument downtime.

qualify To qualify laboratory staff or a subcontractor is to provide evidence of meeting a performance standard for fitness by training skill or ability for a designated purpose. To qualify analytical procedures or computer programs is to provide evidence of performance to meet the required standard criteria.

quality The total integrated program for ensuring the reliability of assurance monitoring and measurement data. Quality assurance is a system for integrating the quality planning, quality assessment, and quality improvement efforts to meet user requirements.

reagent quality An analysis or industry-accepted grade that denotes purity or applicability for application.

regulatory Those methods published or promulgated for laboratory use to meet

procedures the requirement of a law or government rule.

replicate A replicate sample is the 2 nd (or $3 \mathrm{rd}$, and so forth) analysis on the same sample aliquot (not to be confused with a duplicate, which is the 2 nd analysis of a different aliquot) that are taken through the entire sample preparation and analytical process. However, replicate analysis can be run in the same batch at the same time or different batch at a different time from the original sample run. Therefore, the QC sample set up with the replicate analysis can be the same batch or a different batch. Replicate analyses are usually used as confirmatory analyses. Replicate analyses are coded in the data summary report as an " $R$ " on the right side of the sample number column.

representativeness Expresses the degree to which data accurately and precisely represent a characteristic of a population, parameter variations at a sampling point, a process condition, or an environmental condition.

re-run A re-run is a repeat analysis of a previous sample run or set of sample analyses which failed the QC criteria, for example, poor spike recovery, exceeding RPD or other anomalies found in the review the data that failed to meet the quality control criteria. $\mathrm{Re}$-run is the repeat analysis at a different time with new QC. The re-run sample is the aliquot taken from the same original sample and going through the entire sample preparation and analytical process. Re-runs can also be requested by the customer for other reasons than actual QC criteria failures. 
substitution An adjustment in a procedure that has no significant effect on final results. This would clearly be evident in the quality control data associated with the final results.

traceable A document trail that identifies the history of a sample, standard, or other material.

triplicate A triplicate sample is the third aliquot of the same sample that is taken through the entire sample preparation and analytical process. Triplicate sample, like duplicate, is analyzed in the same batch, with the same QC sample set up, and at the same time with the sample analysis. Due to LABCORE's inability to summarize triplicate analyses in the data summary report, triplicate results will be tabulated in the case narrative.

valid Having legal efficacy or force, well grounded, or justifiable being at once relevant, meaningful, logically, and correct, appropriate to the end in view.

validation An act, process, or instance of validating. For data, validation is the process by which the data and quality control information is assessed or compared against the client's requirements.

verification Act or process of verifying. For data, verification is the process of comparing the reported data with the required information.

verifying To establish the truth, accuracy, or reality. 